\title{
Sensor Networks for Active Play in a Massively Multiplayer Online Exergame
}

\author{
By \\ Hannah Johnston \\ A thesis submitted to the Faculty of Graduate Studies and Research \\ in partial fulfilment of the requirements for the degree of \\ Master of Science in Information and Systems Science \\ Department of Systems and Computer Engineering \\ Carleton University \\ Ottawa, Ontario, Canada, K1S 5B6
}

August 2010

(C) Copyright 2010, Hannah Johnston 
Library and Archives

Canada

Published Heritage

Branch

395 Wellington Street

Ottawa ON K1A 0N4

Canada
Bibliothèque et

Archives Canada

Direction du

Patrimoine de l'édition

395 , rue Wellington

Ottawa ON K1A ON4

Canada
Your file Votre référence

ISBN: 978-0-494-71585-7

Our file Notre référence

ISBN: 978-0-494-71585-7
NOTICE:

The author has granted a nonexclusive license allowing Library and Archives Canada to reproduce, publish, archive, preserve, conserve, communicate to the public by telecommunication or on the Internet, loan, distribute and sell theses worldwide, for commercial or noncommercial purposes, in microform, paper, electronic and/or any other formats.

The author retains copyright ownership and moral rights in this thesis. Neither the thesis nor substantial extracts from it may be printed or otherwise reproduced without the author's permission.
AVIS:

L'auteur a accordé une licence non exclusive permettant à la Bibliothèque et Archives Canada de reproduire, publier, archiver, sauvegarder, conserver, transmettre au public par télécommunication ou par l'Internet, prêter, distribuer et vendre des thèses partout dans le monde, à des fins commerciales ou autres, sur support microforme, papier, électronique et/ou autres formats.

L'auteur conserve la propriété du droit d'auteur et des droits moraux qui protège cette thèse. $\mathrm{Ni}$ la thèse ni des extraits substantiels de celle-ci ne doivent être imprimés ou autrement reproduits sans son autorisation.
In compliance with the Canadian Privacy Act some supporting forms may have been removed from this thesis.

While these forms may be included in the document page count, their removal does not represent any loss of content from the thesis.
Conformément à la loi canadienne sur la protection de la vie privée, quelques formulaires secondaires ont été enlevés de cette thèse.

Bien que ces formulaires aient inclus dans la pagination, il n'y aura aucun contenu manquant.

\section{Canadä}


The undersigned recommend to the Faculty of Graduate Studies and Research acceptance of the thesis

Sensor Networks for Active Play in a Massively Multiplayer Online Exergame submitted by

Hannah Johnston

in partial fulfillment of the requirements for Master of Science in Information and Systems Science

Chair, Dr. Howard Schwartz, Department of Systems and Computer Engineering

Thesis Supervisor, Dr. Anthony Whitehead

Carleton University

August 2010 


\section{Abstract}

A sedentary lifestyle is linked to many health problems, including diabetes, heart disease, and obesity. Active games attempt to offer a solution, encouraging players to be more physically active through entertaining media. We examined existing exergames, but found little evidence of quantifiable health benefits and no research to address long-term player engagement. To address this gap, we introduce our own exergame system, the Sensor Network for Active Play (SNAP), and provide experimental results highlighting its capacity to elicit moderate physical activity levels, without negatively influencing player enjoyment. We describe a framework for a massively multiplayer online exergame (MMOE), combining persuasive technology and massively multiplayer online games for long-term engagement. We revisit the framework to assess the use of the SNAP system in an MMOE. There are several technical and usability challenges in the development of an MMOE, which we address, including pose selection, training, recognition, and presentation methods. 


\section{Acknowledgements}

I am extremely thankful to my supervisor, Dr. Anthony Whitehead, whose guidance and feedback have been instrumental in this thesis and the completion of my academic program. I would like to extend thanks to Dr. Robert Biddle, who provided valuable insight and sparked my interest in persuasive technology and other relevant topics in human-computer interaction. This thesis has also benefitted greatly from work done with Dr. Jo Welch and Nicole Nixon at Dalhousie University.

Thank you to my peers, Kaitlyn Fox, Nick Crampton, and Joe Tuen, for making the development of the SNAP system possible and endlessly entertaining.

This work could not have been completed without help from the many participant volunteers who graciously gave their time to train and replicate poses with the SNAP system, providing valuable data and feedback.

I am grateful to Carleton University and NSERC for providing me with funding through the completion of this work.

Finally, I would like to thank my family and friends for their continued support and encouragement. Thanks especially to Mom and Dad for their patience, clever suggestions, and for providing me with good food throughout the process. 


\section{Table of Contents}

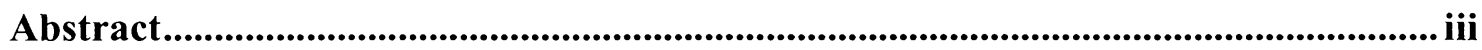

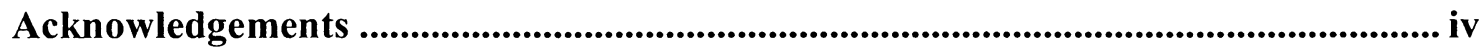

Table of Contents ..................................................................................................................... v

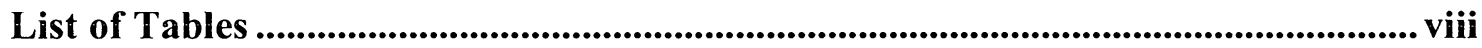

List of Figures...................................................................................................................... ix

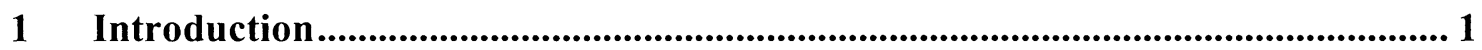

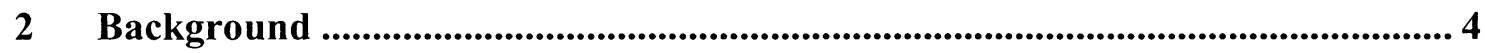

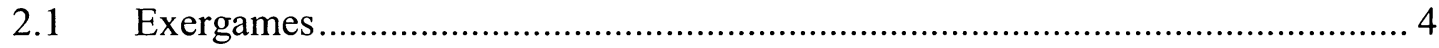

2.1.1 Commercially Available Exergame Systems.................................... 4

2.1.2 Exergame Prototype Technologies ................................................ 5

2.1.3 Types of Exercise in Exergames ......................................................... 6

2.1.4 Qualitative Exergame Research .................................................... 7

2.1.5 Quantitative Exergame Research ....................................................... 8

2.1.6 Physical Input System Requirements.................................................... 10

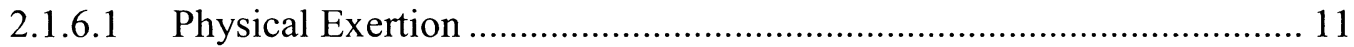

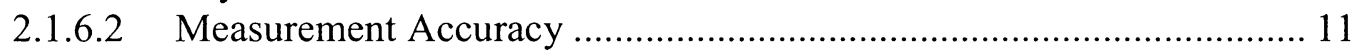

2.1.6.3 Comfort of Wearable Sensors and Markers ....................................... 13

2.1.6.4 Accessibility and Convenience ........................................................ 14

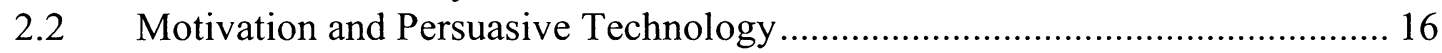

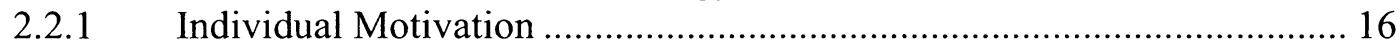

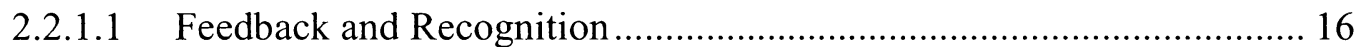

2.2.1.2 Challenge and Flow .................................................................. 18

2.2.1.3 Tailoring and Customization...................................................... 20

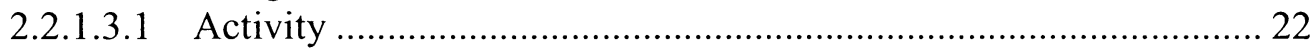

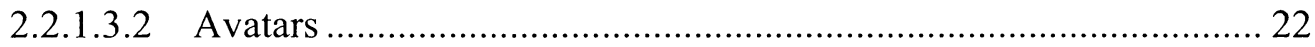

2.2.1.3.3 Music................................................................................ 23

2.2.1.3.4 Connection to a Network ..................................................... 24

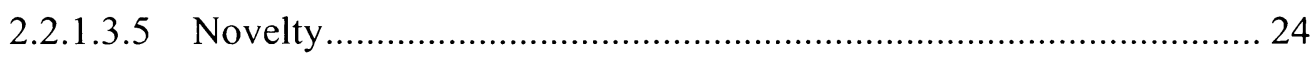

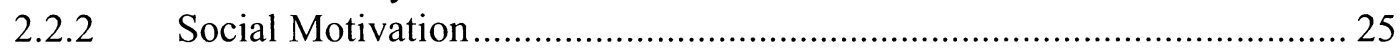

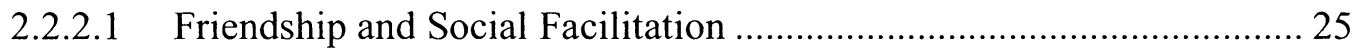

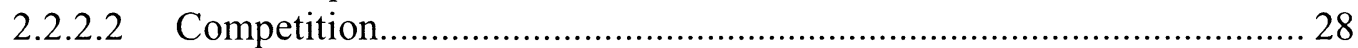

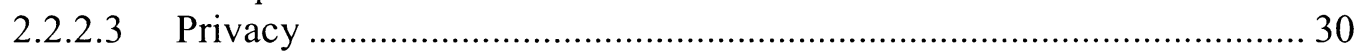

2.3 Massively Multiplayer Online Games ..................................................... 32

2.3.1 MMOG History, Popularity, and Player Demographics.......................... 32

2.3.2 MMOG Genres ............................................................................... 33

2.3.3 Virtual Game Worlds, Avatars, and Content ......................................... 33

2.4 The Sensor Network for Active Play (SNAP) System .................................. 35

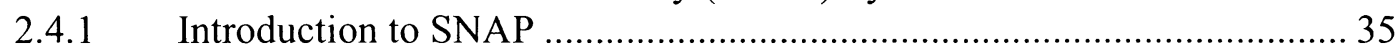

2.4.2 Design and Implementation of the Sensor Network ............................... 36

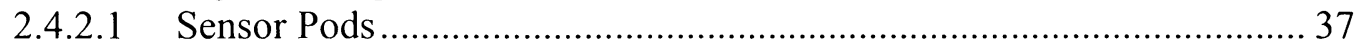

2.4.2.2 Sensor Pod Placement.................................................................... 37 


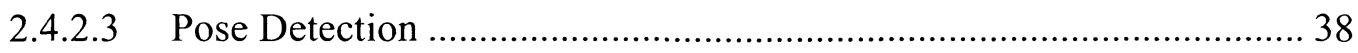

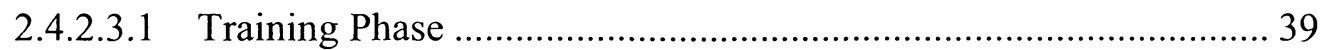

2.4.2.3.2 Recognition Phase...................................................................... 40

2.4.3 Experimental Results with SNAP …………………………………..... 41

2.4.3.1 Optimal Number of Data Samples to Include in Training .................... 47

2.4.3.2 Strictness in Training Poses ............................................................... 48

2.4.3.3 Technical Limitations of Speed ........................................................... 49

2.4.3.4 Quantitative Exercise Benefits......................................................... 50

2.4.3.5 Pose Success and Physical Exertion ..................................................... 53

2.4.4 Game Design and Development with SNAP ......................................... 54

2.4.4.1 Persuasive Strategies.......................................................................... 59

2.4.4.2 Player Enjoyment............................................................................ 59

2.4.4.3 Pose Selection ................................................................................. 59

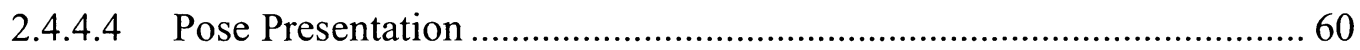

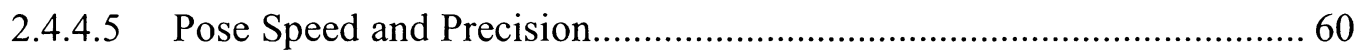

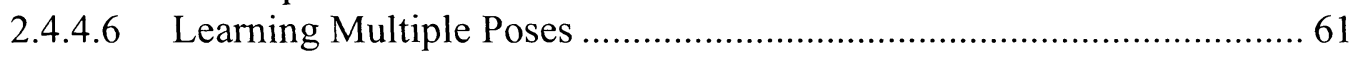

3 Using SNAP in a Massively Multiplayer Online Exergame.................................. 62

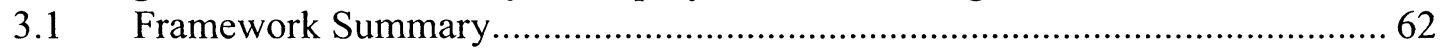

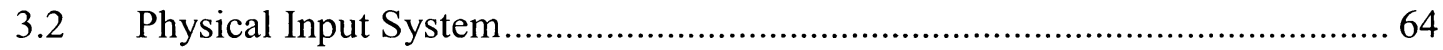

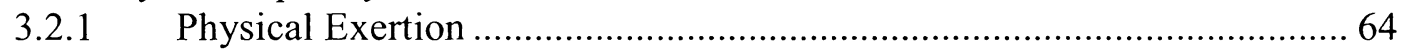

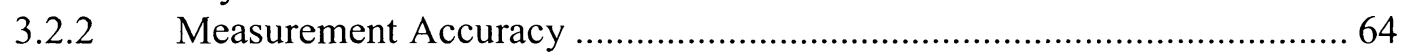

3.2.3 Comfort of Wearable Sensors and Markers................................................ 65

3.2.4 Accessibility and Convenience ................................................................ 67

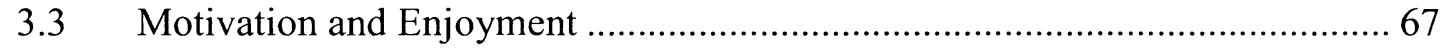

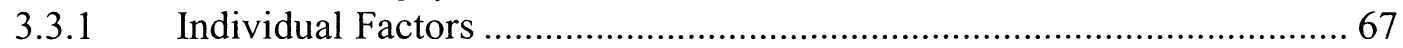

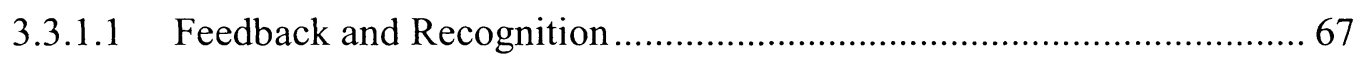

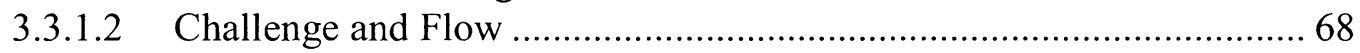

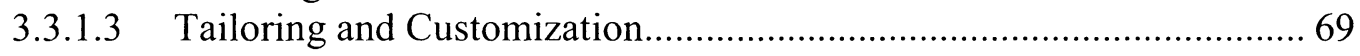

3.3.1.3.1 Activity ............................................................................... 70

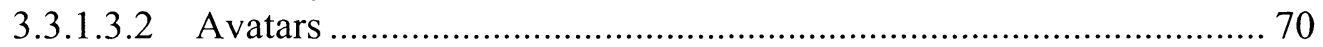

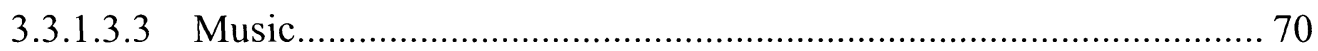

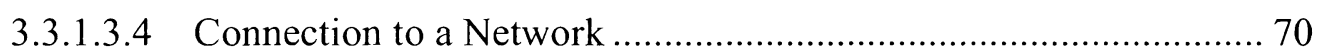

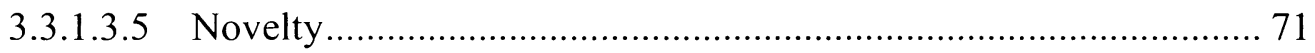

3.3.2 Social Factors ............................................................................. 71

3.3.2.1 Friendship and Social Facilitation ................................................... 71

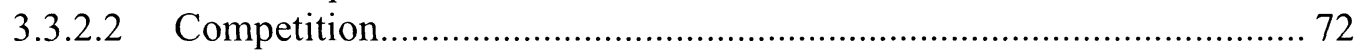

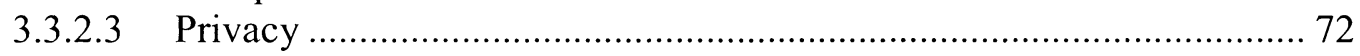

4 Development of a Customizable Dance Pose Creation System............................ 74

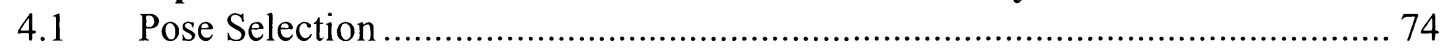

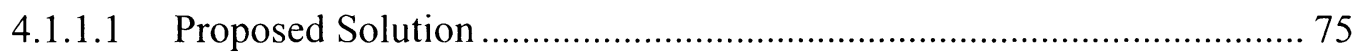

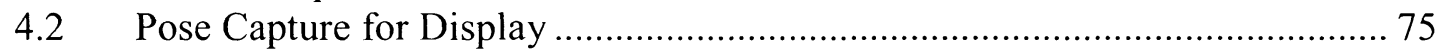

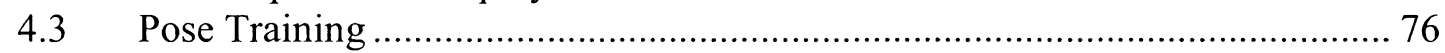

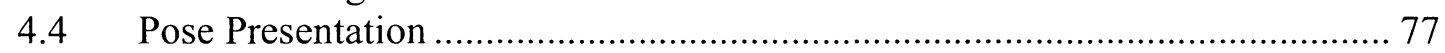

4.4.1 Pose Presentation Testing ................................................................. 78

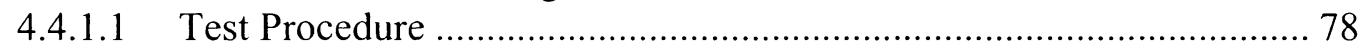

4.4.1.2 Test Conditions ................................................................................ 79 


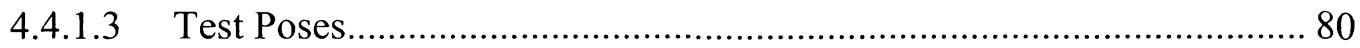

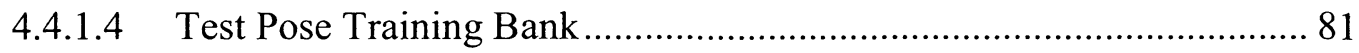

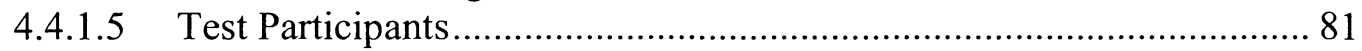

4.4.1.5.1 Young Adult Participants.......................................................... 81

4.4.1.5.2 Older Female Participants...................................................... 82

4.4.1.6 Pose Presentation Results ............................................................. 83

4.4.1.6.1 Effect of Presentation Method on Speed ..................................... 83

4.4.1.6.2 Effect of Presentation Method on Accuracy ................................. 86

4.4.1.6.3 Effect of Presentation Method on Perceived Difficulty................. 89

4.4.1.6.4 Pose Presentation Feedback Functions ......................................... 91

4.4.1.6.5 Baseline Sensor Error .............................................................. 91

4.4.1.6.6 Dynamic Pose Presentation.......................................................... 92

4.4.1.7 Impact of Other Factors on Pose Success .......................................... 93

4.4.1.8 Challenges Facing Older Participants .............................................. 94

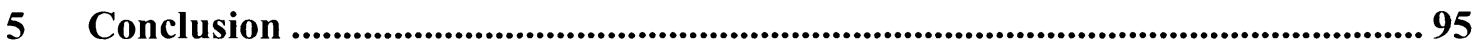

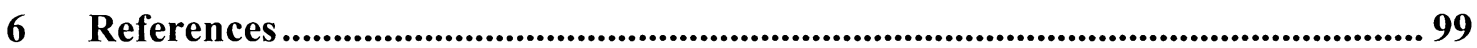




\section{List of Tables}

Table 2.1 Pose confusion matrix for training data of seven subjects............................... 44 Table 2.2 Average pose multiplier factors for single and multiple training data set sizes 44 Table 2.3 Effect of multiple training data samples on recognition rates .......................... 44

Table 2.4 Mahalanobis distances, with networks of four and eight sensors..................... 45

Table 2.5 Participant Stats: Age, Height, Weight and BMI........................................... 51

Table 3.1 MMOE Framework summary: physical input system ....................................... 63

Table 3.2 MMOE Framework summary: motivation and enjoyment requirements.......... 63

Table 4.1 Comparison of automated and user-controlled pose selection methods........... 75

Table 4.2 Comparison of poseable avatar and image-based pose selection methods....... 76

Table 4.3 Young adult participant characteristics..................................................... 82

Table 4.4 Older adult female participant characteristics ............................................ 82

Table 4.5 Statistics describing speed by presentation method....................................... 84

Table 4.6 Average time to pose, broken down by individual pose................................... 85

Table 4.7 Statistics describing accuracy by presentation method................................... 86

Table 4.8 Average Mahalanobis distance, broken down by individual pose................... 87

Table 4.9 Average perceived mental and physical difficulty by presentation method ..... 90

Table 4.10 Speed of female participants of different ages................................................ 94

Table 4.11 Accuracy of female participants of different ages .......................................... 94 


\section{List of Figures}

Figure 2.1 Menu for the Nintendo Wii Mii avatar setup ............................................ 23

Figure 2.2 Plastic pods used to encase accelerometers with straps ............................. 37

Figure 2.3 The placement of the 4 sensor pods ........................................................ 38

Figure 2.4 The eight poses, inspired by Michael Jackson's Thriller ........................... 42

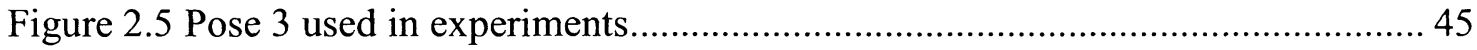

Figure 2.6 Trend lines for the learning curves of seven subjects............................... 46

Figure 2.7 Convergence of distances as the training data set increases........................... 48

Figure 2.8 Effect of speed on success rate for a single person ................................. 50

Figure 2.9 Mean heart rate for each intervention................................................ 52

Figure 2.10 Mean energy expenditure for each intervention ...................................... 53

Figure 2.11 Heart rate increases as the ability to successfully duplicate poses increases 54

Figure 2.12 Users playing Track Heroes Hurdles (Left) and a game screenshot (Right). 55

Figure 2.13 Screen shot of Posemania dancing game.............................................. 56

Figure 2.14 Screen shot of Posemania dancing game................................................ 57

Figure 2.15 Dance Fighter game play ............................................................... 58

Figure 3.1 Sensor rotation angle as the pod moves from wrist to forearm ..................... 66

Figure 4.1 Presentation conditions (left to right) direct, mirrored, mirrored and back, mirrored and side, and mirrored with feedback ...................................................... 80

Figure 4.2 Practice pose (pose 0 ) and five poses for presentation method testing .......... 81

Figure 4.3 Speed by presentation method........................................................ 84

Figure 4.4 Breakdown of the speed required for each pose....................................... 85

Figure 4.5 Mahalanobis distance by presentation method........................................... 87

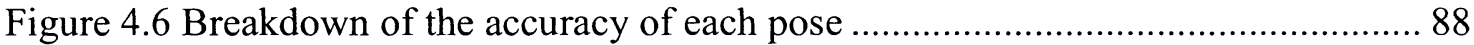

Figure 4.7 Pose 2 from the front (mirrored), side, and back views .............................. 89 


\section{Introduction}

Obesity has become a worldwide epidemic, with physical inactivity contributing to approximately two million deaths a year [1]. There are growing concerns over the increasingly sedentary lifestyle of many industrialized societies, as it is a major underlying cause of disease, disability, and death [2]. There has been a dramatic rise in the prevalence of type II diabetes, heart disease, and some cancers [3], with problems like depression, anxiety and mental illness on the rise [1]. In addition to the many adverse health consequences, obesity also has a corresponding social stigma [4]. These health consequences not only affect individuals, they also put pressure on health care systems [3]. Regular physical activity is critical to everyone's physical and psychological health, regardless of his or her weight. An active lifestyle reduces risk of disease and makes the body stronger, fitter, and more flexible. Despite the many positive physical and psychological effects [5], many people still fail to meet recommended levels of moderate physical activity [6]. Children and adolescents are particularly vulnerable, devoting more time to video games and other sedentary entertainment. These activities are often addictive and self-reinforcing [7], making the desire to lead a more active lifestyle increasingly difficult, a fact that is only compounded by economic, psychological, and social challenges [4].

These increasing health concerns and the growing popularity and commercial success of the Nintendo Wii have sparked interest in physically active games, commonly referred to as exergames [8]. While few people would dispute the potential of these games to encourage physical activity, little research has investigated long-term or measurable 
benefits of exergames. There is an increasing number of exergame prototypes being developed, including our own, full-body dance-based game, however, they rely heavily on interface novelty to motivate players. In addition to more rigorous physical requirements, exergames must be supplemented with individual and social motivational elements to elicit maximum health benefit. Massively multiplayer online games (MMOGs) may provide a viable solution, through strong social strategies and a constant influx of player-generated content; however, moving dance pose creation and training online introduces a new set of challenges. Capturing and presenting 3-dimensional, fullbody poses is most successful when facilitated in-person, something that is not possible in the MMOG context. This thesis investigates exergame customization strategies, which could ultimately be used without intervention in an online world. The main challenge is to design intuitive and useable methods for pose capture and subsequent presentation of user-generated dance poses.

We believe that the popularity of exergames is due in large part to the novelty of their interface. While this novelty may initially act as a strong motivator, it is our contention that games with leverage social ties, through competition and friendship, will more effectively encourage long-term player engagement. MMOGs, such as World of Warcraft, have proved successful in their ability to maintain player interest, in large part by fostering a sense of commitment and loyalty among player groups of friends and online acquaintances [9]. This thesis presents a new concept, merging massively multiplayer online games, exergames, persuasive technologies, and the proven SNAP input system $[10,11,12,13]$ in what we coin as a Massively Multiplayer Online Exergame, 
or MMOE. We provide an overview of initial MMOE requirements and address issues that arose in the design of this framework. While the outline of MMOE elements has not been comprehensively developed or tested, this work explores implementation trade-offs for a subset of the requirements, addressing the core issues of pose selection and presentation.

To get a sense of the current state of the research and relevant areas of development, we begin with a survey of exergame systems, motivation and persuasive strategies, and massively multiplayer online games. An introduction to our own SNAP exergame system follows, along with an overview of some of the major system and game design issues. Next, we propose a framework for a massively multiplayer online dancing game and examine several key customization components. We discuss the SNAP system as an input system for a massively multiplayer online exergame. A summary of test results follows, with suggestions for effective pose selection, capture, training, and optimal methods of pose presentation. We conclude with a summary of key conclusions and a discussion of opportunities for future work. 


\section{Background}

In this thesis, we propose combining exergames, persuasive technology, and massively multiplayer online games to deliver a more entertaining gaming experience that will consequently be a more successful long-term solution to physical inactivity. This section provides an introduction and overview of relevant existing research on exergames, persuasive technology, and massively multiplayer online games.

\subsection{Exergames}

Exergaming leverages entertaining and engaging aspects of traditional gaming to encourage participants to be more physically active. These games address a number of different user groups and their unique needs, while promoting active lifestyles through a variety of different strategies and devices. Commercially available and research prototype systems are described next.

\subsubsection{Commercially Available Exergame Systems}

There is a wide variety of commercially available exergame systems. Some of the earliest exergames make use of stationary bicycles $[14,15,16,17]$, including the TACX Fortieus Trainer $[18,19]$ and the Virku, Virtual Fitness Centre $[14,19,20]$ cycling games. Camerabased systems are also common, with the Sony EyeToy appearing most frequently in the literature [21]. The EyeToy is an add-on device for the PlayStation 2. It uses computer vision and gesture recognition as input. Many games have been developed for the system, including Play, the first game created for the EyeToy accessory; Groove, a dancing game; AntiGrav, a hoverboard game targeted at serious gamers; and Kinetic, an exercise 
program, developed in collaboration with Nike Motionworks [22]. The successor of the EyeToy is the PlayStation Eye, for PlayStation 3 [23].

Game boards and pads are another popular input method for active games. Early commercial game pads include the Nintendo Power Pad and the Amiga Joyboard [19]. More recently, XaviX developed the J-Mat [24] and Nintendo created the Balance Board for the Wii [25]. Konami's Dance Dance Revolution, arguably the most extensively researched exergame, utilizes a dancing pad, labelled with a series of arrows. Andamiro's "Pump it Up" [22] and "In the Groove" from Roxor Games [21] use a similar button pad input system.

The Wii, which leverages multiple different sensor types, is commonly studied and evaluated. For example, in one study, the health benefits of the system were compared to those elicited by traditional sports, including tennis, baseball, bowling, golf, and boxing activities [26]. Wii Fit is an extension of the Wii system, using the Wii Balance Board accessory. The game includes many different activities, including yoga poses and pushup exercises [19]. Wii remotes, or Wiimotes, have also been used in the development of other exergame prototypes $[27,28]$. Some of these prototype systems are described in the following subsection.

\subsubsection{Exergame Prototype Technologies}

Accelerometers are frequently used in on-the-body systems to evaluate the amount of physical activity $[29,30]$ and, in some cases, they are also used as game input or to infer the activity type [31]. Pedometers are typically accelerometers, used in exergames in 
similar ways $[4,32,33]$. Heart rate is frequently monitored and used as a method of game input and control [34,35], but it is also used to evaluate player exertion or dynamically adjust the level of difficulty in the game [17]. Many active game prototypes leverage the ubiquity and convenience of mobile devices, for example Fish'n'Steps [4] and UbiFit Garden [31]. GPS is also becoming increasingly popular and widely used among mobile exergame prototypes [20,36,37].

Exergame systems vary significantly in the amount of exercise and entertainment they provide. NEAT-o-Games [38] and AstroWheelie [27] integrate a physical activity element into traditional games, while others attempt to add game-like incentives to exercise. For example, media stations provide motivation through television or traditional video games, while participants walk on a treadmill $[39,40]$ or pedal a stationary bicycle [15]. Augmented reality (AR) has also been used to bridge the gap between real-world exercise and digital entertainment elements. AR game prototypes include Laser Tag [41]; Age Invaders, a rocket-shooting game [42]; Paranoia Syndrome, in which players physically walk around a rooms while interacting with a personal digital assistant (PDA) and physical objects with radio-frequency identification (RFID) tags; and Human Pacman, in which players act as Pacman and the ghosts [14].

\subsubsection{Types of Exercise in Exergames}

Perhaps largely due to their fast pace and the widespread popularity of Wii Sports and Dance Dance Revolution, boxing [43,44] and dance [28,45,46] games are most commonly studied within the exergame literature. Cycling $[16,17,47]$, bowling $[24,48]$, and tennis [37] are also popular among the exercise types in the games surveyed. Several 
studies have examined active games using movements based on boxing variations, for example such as boxercise [34]. Bowling [24] and table tennis [49,50] games have also been examined.

\subsubsection{Qualitative Exergame Research}

Current exergame research is predominantly qualitative. To make exergames a viable solution to rising health concerns, researchers need to investigate ways of enhancing player engagement, following the initial novelty phase of traditional exergame systems. Many exergame prototypes exist; but evaluation and analysis of the systems is limited. The vast majority of the qualitative research involves the design of new exergame system prototypes. This work often describes the design process [20,51], iterations [32,35], and decisions [52]. Several papers provide generalizations for game design guidelines $[18,20,53]$ and principles [54]

Much of the qualitative exergame research and system development targets children, for example through sports $[43,55]$, outdoor activity [35], or music and dancing [32]. Several papers also specifically address the needs of teenagers [3], adolescents $[46,48,56]$, and adults $[15,17,57]$. One study targeted multi-generational families, including the elderly [42], and several papers attempt to address accessibility of exergames [3,27,58].

Qualitative studies suggest a broad range of positive side effects that may be attributed to active games. Research suggests that exergames can be used to improve participants' enthusiasm for physical activity [59], rhythm [28,45], self-esteem, and social skills [59]. To date, there have been no long-term exergame studies, so the sustainability of game 
enjoyment, health improvement, and any positive behaviour change remains unknown. Both commercial systems and research prototypes rely heavily on the novelty of the interface to draw in participants. While this approach may be highly successful initially, over time, players lose interest and the games no longer serve as a motivational tool.

While the vast majority of exergame research involves system analysis and comparison, some studies use questionnaires [41,45] and ethnographic research methods [51] to evaluate commercial exergames and research prototype games. There are also several comprehensive exergame system and literature surveys available $[18,60,61]$.

\subsubsection{Quantitative Exergame Research}

Of 15 quantitative exergame studies, six tested games for the Nintendo Wii $[26,48,56,62,63,64]$, three examined exertion with Sony's EyeToy games $[65,66,67]$, and six used Konami's Dance Dance Revolution (DDR) $[16,33,39,62,66,68]$. One study compared exertion level of participants at two different levels of game difficulty (beginner and basic) [62]. Another DDR study found that experienced players exerted more energy that inexperienced participants [66]. Some quantitative work has been done to measure exertion of walking briskly while watching TV or using a media system $[24,39,65]$.

Children and adolescents are a popular target audience for quantitative exergame studies, leaving a gap in research addressing the needs of older populations. The majority of the quantitative studies involved participants with mean age under 20 $[16,24,33,48,56,62,66,67,69]$. Several studies measured exertion in people with mean age 
over 20 [16,26,63,64,66]. While Siegel et. al. grouped participants into two groups: under 19 and 20 or over [16], only one study explicitly mentioned participants with a mean age over 30 [64]. No quantitative studies could be found measuring energy expenditure among middle-aged or older adult populations.

Several studies conducted separate data analysis of male and female participants $[16,62,63]$ and one study examined male-only players [66]. Quantitative studies made health and fitness comparisons on a number of variables, including differences in energy expenditure with human and computer opponents [63] and lean versus overweight players [39]. The quantitative studies examined ranged between 7 and 33 participants $[33,66]$.

All of the systems examined elicited average heart rates and energy expenditures above rest within their own studies. All of the systems, except XaviX Bowling, resulted in energy expenditures above an average rest value of $1.91 \mathrm{Kcal} / \mathrm{min}$, calculated using rest data from seven studies $[24,39,48,56,64,67,69]$. Not surprisingly, the results show that exergames requiring any type of body movement result in increased energy expenditure over sedentary activities that do not require movement. While many of the games surveyed may not provide users with all of their recommended daily exercise, exergames still provide some health benefit over sedentary, screen-based entertainment.

The more energy intensive systems usually involve predominantly leg movements, for example DDR [13,21,52,58,74,75], 3-Kick [16], and bicycling games [16]. This finding 
is consistent with our hypothesis that games enforcing full-body movement will result in exercise that is more rigorous. Upper-body movement is limited in the majority of the games identified, which suggests that leg movement alone may be sufficient. The majority of the systems do not address cheating or precision issues, such as improper or inaccurate activity performance, and instead assume proper play, suggesting a need for systems that can more accurately detect full-body activities.

There are a number of factors that influence participants' level of exertion, including physical characteristics, motivation, and experience. There is a strong correlation between heart rate and energy expenditure [70], suggesting that complex procedures for measuring energy expenditure are not necessarily required in order to estimate the physical effectiveness of an exergame [13]. Digital heart rate monitors are a comparatively convenient and economical alternative to more sophisticated metabolic measures.

\subsubsection{Physical Input System Requirements}

Exergames rely on some form of physical exertion from the player as part of the game interaction. Considerations for physical input systems include the amount of physical exertion they encourage, the degree of system measurement accuracy, the comfort of wearable components, and the overall accessibility and convenience of the game system. We discuss these aspects in turn. 


\subsubsection{Physical Exertion}

Exergames provide users with varying levels of physical exertion and intensity. Existing quantitative research suggests that the majority of exergames provide participants with only low to moderate intensity workouts [56]. At a minimum, games should provide players with the option to participate in ways that are more or less physically demanding to meet individual participant needs. To ensure the effectiveness of future games, developers may consider incorporating heart rate monitors as a means of monitoring player exertion.

\subsubsection{Measurement Accuracy}

There is some debate as to the importance of accuracy in exergames. While some players are willing to suspend their disbelief and overlook shortcomings, many participants find inaccuracy distracting and frustrating [19]. As the novelty of early exergame interfaces gradually wears off, players expect more accurate system response. One solution has been to address the shortcomings of input systems by supplementing them with additional sensor types [71]. The Nintendo Wii Remote and Nunchuk are popular, accelerometerbased gaming peripherals. The Wii addresses limitations with infrared (IR) LEDs in the console's Sensor Bar. Nintendo has since improved accuracy and realism, with the Wii MotionPlus accessory. It uses an angular rate sensor to supplement the accelerometer and Sensor Bar capabilities of the Wii Remote, enabling controller motions to render consistently on the screen in real time [72]. Camera or computer-vision-based input systems may rely on other technologies to improve their accuracy. Sony's motion controller captures movement based on visual detection of a hand-held baton, using 
ultrasound to improve accuracy. Depth cameras can provide 3-dimensional images of scenes, permitting full-body tracking. Microsoft's Project Natal [73] accomplishes something similar by shining pulses of IR light into the scene, and measuring the reflections of this light, thus eliminating the need for a controller [71]. The vast range of input types available among current commercial exergames and game prototypes suggests that future games will benefit less from additional possibilities in active input types than from an improvement of detection accuracy and realism. Project Natal is capable of distinguishing the player's body position in space, providing more accurate stance input than is possible with pressure sensors, used by a device like the Wii Balance Board [71]. However, Project Natal suffers from many of the same problems that earlier camera-based systems do. For example, some have claimed that the system has trouble recognizing gestures of players with darker skin tones [74,75].

Many persuasive technologies for fitness rely on pedometers, but unfortunately, they often provide deceptive or inaccurate measurements and grossly under-estimating the exertion required for activities like cycling, swimming, and strength training [53]. These recognition errors may cause players to miss out on in-game credit or recognition. Alternatively, the systems may require manual recording of activities. In one system, manual additions represented a whopping $61 \%$ of all activities performed, suggesting a need for more accurate recognition systems [31]. Pedometers also fail to take into consideration subtleties like speed and stride length. Technologies that are more sophisticated may be able to address this issue, detecting and measuring a wider variety of physical activities that are common for the target audience. Nevertheless, measurement 
alone often fails to capture sufficient information. One way to address this issue is to allow users to edit and supplement automatic sensor measures with information and descriptions [53].

\subsubsection{Comfort of Wearable Sensors and Markers}

For input systems that make use of wearable sensors or markers, it is important to consider user comfort, both in terms of physical attributes like size, weight, and placement of sensors, as well as psychological factors, such as the potential to cause the wearer undue embarrassment.

The position on the body and ease of adjustment should permit users to accurately attach sensors quickly, correctly, and independently. Removal should also be quick and easy. Sensors and markers should be as unobtrusive as possible as the size, shape, and weight of devices can greatly influence the overall user experience. Designers should make sensors and markers as lightweight and inconspicuous as possible. Smaller, wireless equipment helps to ensure that player movement remains unrestricted. Sensors and markers must be comfortable to wear, without any pieces that jab, poke, or otherwise irritate the wearer. Exercise conditions can be extreme, so sensors and markers must be capable of withstanding exposure to sweat, motion, bumps, and even being sat on. Providing replaceable components may be one solution to the problem of wear and tear. As players often share game systems, it is reasonable to expect that several different users may wear sensors. Any device that is exposed to skin or sweat should therefore be washable. 
Placing multiple sensors or markers all over the body provides accurate activity measurements; however, they may quickly become annoying to place and to wear. Limiting the size and number of sensors may reduce player embarrassment and contribute to overall psychological comfort. Camera-based motion capture systems, such as those used in animation, require participants to wear full-body spandex suits, with dozens of reflective markers attached. While these systems can record 3-dimensions of movement with a high degree of accuracy, they are impractical for exergame applications. Exergame users should be free to play in whatever clothing they are comfortable; spandex should be an option, not a requirement.

\subsubsection{Accessibility and Convenience}

The convenience and accessibility of exergames provides a valuable feature compared to many other exercise programs. The ubiquity of technological measuring devices allows people to skip the gym and instead workout whenever and wherever is most convenient

[2]. The ability to participate from the convenience of home may also improve exercise adherence [26]. Participants should be able to engage with the game at any time of day or night, from the comfort of their own living rooms. An online system helps ensure the $24 / 7$ availability of the system. Players can either compete against someone in the same room, or from anywhere else in the world. Systems should be safe and easy to use in the home environment, without extensive setup or adjustment of the living space. 
While some of the exergame research prototypes involve in-person training, workarounds, and other Wizard of $\mathrm{Oz}^{l}$ techniques, many commercial systems provide users with an easy-to-use, plug and play setup. It is important to consider the type of interface when developing exergame requirements. Mobile devices are impractical if participants are required to input a lot of text information. Traditional game controllers make this input even more challenging.

Exergames should accommodate a wide range of player environments, independent of factors such as lighting, background, and space constraints. It is unreasonable to assume that players will have access to a dedicated, gym-like space, in which to play the games. While players are responsible for removing physical obstacles, exergames should not require an excessively large area for movement and play. Input devices should be wireless whenever possıble, but it is especially important that cords not restrict or interfere with body movement, as they may be both annoying and potentially dangerous. It is also important to consider players' access to existing equipment, such as televisions, computers, or other necessary hardware, that is not included as part of the game system. Some exergames require setup of cameras, IR sensors, or other devices. If applicable, additional equipment should accommodate a variety of placement locations, and provide players with adequate tools and setup instructions. Exergames should require the least amount of extraneous devices, sensors, and equipment possible.

1 The term "Wizard of Oz" originated from John F Kelley to describe a research experıment in which subjects interact with a computer system that subjects believe to be autonomous, but which is actually being operated, or partially operated, by an unseen human being 


\subsection{Motivation and Persuasive Technology}

Persuasive technology is "technology that is designed to change attitudes or behaviours of users through persuasion and social influence, but not through coercion" [76]. Technology can persuade in many different ways, including embedded reminders, visualizations, and praise. Persuasive technology can also provide strategies to track behaviour and highlight consequences, allowing users to better project future scenarios. Persuasion techniques are most effective when they are interactive, giving games a strong advantage over other methods. Persuasive technologies are effective in large part because they can adjust their influence tactics as the situation evolves, based on data received about the users' interests, needs, and situation. Many factors contribute to player enjoyment of games. While some elements of game play motivate individual players, social strategies may be especially successful for persuading and encouraging participants. In this chapter, we discuss motivation and persuasive strategies, broken down into individual and social techniques.

\subsubsection{Individual Motivation}

Game designers can impact the enjoyment and motivation of individual players through appropriate feedback and recognition, balancing skill level and challenge, and through the inclusion of personalized and customization elements.

\subsubsection{Feedback and Recognition}

One of the key design requirements for technologies that encourage physical activity outlined by Consolvo et al. is to "give users proper credit for activities" [53]. People are more motivated to exercise when they receive credit and recognition for the activities 
they perform. According to Fogg, "by offering public recognition (individual or group), computing technology can increase the likelihood that a person or group will adopt a target attitude or behaviour" [76].

Ongoing feedback helps keep players motivated and engaged throughout the game [77]. Incorporating self-monitoring and feedback strategies into an exergame in different ways provides players with more information about their activities and motivates them to expend more energy. Providing players with appropriate credit and recognition helps motivate players to reach their goals. In Houston, a persuasive technology mobile phone prototype encourages activity by counting steps. Participants received motivation and recognition through stars and congratulatory messages from the system. Proper credit is even more important when score counts are pooled or shared between players [53]. Game play logs and histories provide users with even more information for self-monitoring. Feedback for players might include game scores, play duration, heart rates, and even some estimate of fitness level over time.

In systems that measure physical activity, it is important to give users appropriate credit for their actions [53]. Participants are frustrated and disappointed when the device fails to detect a specific activity it was trained to infer. These failures lead users to question the reliability and purpose of the device [31]. Users feel cheated when the system is wrong and they cannot do anything to fix it. One solution to inference error is to allow users to add, edit, and delete recorded data. It gives participants a more complete and accurate historical record of their activity and improves credibility of an imperfect system. Many 
commercial products focus instead on cheating, by taking over complete control of the data. Further study is necessary to explore the role of data manipulation in systems that share with others or promote competition [31].

A heart rate monitor can provide additional opportunities for data collection and feedback. The game interface might include a number of glanceable icons, representing heart rate, level of exertion, or estimated calories expended. Text, icons, and highlighted body parts may vary in colour, depending on how close the user is to his or her target heart rate. For example, red could indicate being far from the goal state; yellow, close; and green, within the target range. Auditory suggestions and musical cues can encourage players to reach their target heart rates or levels of exertion. It is more difficult to integrate self-monitoring strategies into multi-player versions of the game. Feedback must be specific to each individual, but if players are co-located, they look at the same screen. A split-screen could address this issue and better accommodate players of varying experience levels. Another solution might be to attach tactile feedback devices to wearable sensors, providing direct, personal feedback, without disrupting other players.

\subsubsection{Challenge and Flow}

The goal within an interactive game is the central motivating factor, especially if the goal is challenging and the player has to strive to meet it [78]. Players immerse themselves more deeply in challenging games and find them more rewarding when the challenge is difficult, there is a greater possibility of failure, or if there is more than one path to success [79]. A challenging goal is a major attraction for players, particularly adolescents [80]. Some players also use challenging games to push themselves further, accomplishing 
more and raising their skill level. The desire to beat the level or complete the game motivates players and can be addictive. Some players enjoy gaining confidence through playing a small, familiar set of games that they are able to master [81].

The appropriate level of challenge can be one of the most important ways to achieve a fun and meaningful play experience [54]. Csikzentmihalyi introduced the concept of flow: a sense of pure concentration and immersion experienced when a participant is engaged in a challenging activity [82]. Flow can occur in work or play, whenever players engage in activities that require concentration and control. People focus intensely on the activity and may lose their sense of time and place [83]. Maintaining game flow is particularly important within the context of exergames and is widely recognized among game designers and developers $[18,19,54]$. Any interruption can shift player focus from the enjoyable game to the physical activity required [7]. Players who achieve a flow state have more desire to continue playing and meet subsequent game challenges [54]. Game attractiveness increases when perceived skill level balances perceived level of challenge or difficulty. Similarly, game effectiveness improves with the physical balance of tolerating exercise and intensity [18]. To encourage flow, games should be sufficiently challenging to elicit players' best efforts, yet not so difficult that challenges cannot be met [83]. Game play can supplement traditional exercise by providing many of the qualities necessary for flow, including immersion, realism, interactivity, exhilaration, and graded challenges [69]. 


\subsubsection{Tailoring and Customization}

Information that technology provides its users is more persuasive if it is tailored to the individual's needs, interests, personality, and usage context. People typically pay more attention when it appears as though content is personal and specific to them. Designers can make technology more persuasive by tailoring to what users are doing, who they are with, what mood they are in, and what they have recently achieved [76].

In an effort to personalize the gaming experience, we can tailor or customize games according to player needs. In this work, we refer to tailoring as modifications made by the system in order to better meet the needs of the player, while customization, in turn, refers to adjustments to the game made by players themselves. Interactive games rely on user input in the form of actions and messages to generate an appropriate response. Successful games tailor responses and feedback to the specific abilities and needs of individual players. Tailored elements provide players with better support and give users a greater sense of control and active involvement [79]. Tailoring and customization often act as effective persuasive tools. The more personal the experience, the more engaged, and consequently physically active, the player will be. Several basic methods of game customization are easy to implement. We can modify the visual appearance of player avatars, the music, and the level of difficulty in the game to provide a more enjoyable experience. A dance-based exergame could permit further customization by allowing

players to create their own dances and select their own dance poses for themselves and others to perform. 
To enhance the persuasive power of technology-based physical activity interventions, programs should be customizable, accommodating the underlying cognitions of inactive users. Technology can provide user-tailored interactions by learning from past interactions and personalization, or through data collection and analysis $[1,4]$. In one study, a shortened version of the Big 5 personality test helped select the ordered list of games presented to players, based on individuals' introversion or extroversion. The system provides users with a wide variety of games, giving players control and ability to personalize their activity schedule [3]. Acceptance of technological devices may depend on the user. Evaluation of player differences is necessary to model more effective, dynamic strategies for different user types [7]. An examination of internet-based persuasive technologies for fitness found that individually tailored interventions encouraged more participants to meet recommended physical activity requirements than the control group; however, activity monitors measured no difference in actual activity [5]. So while tailoring may make users feel more motivated to exercise, it is unclear whether the principle is powerful enough to motivate any real behaviour change, and further study is required.

Customization helps game designers accommodate a wider range of personal styles, tastes, and interests. The more flexible the system, the more diverse the potential audience, and consequently the more users that may want to play the game. This is particularly important in the context of MMOGs, as a large user base is critical to the success of the game. Player activities and avatars can enhance the personalized 
experience. Music selection, connection to a network, and novelty can also be used as persuasive tailoring strategies that lead to a more engaging gaming experience.

\subsection{Activity}

Physical exertion in games is encouraged when players have the opportunity to participate in an activity of their choosing. In a dance-based exergame, personal taste and freedom of expression are especially important. Games should provide players with the system functionality necessary to recognize whatever dance moves the player enjoys, from break dancing to ballroom dancing to classical ballet. Different cultures often have their own styles, both in traditional and informal dance. Accommodating a wide variety of moves is critical to the success of a massively multiplayer online exergame (MMOE). Not only will more people enjoy a game that caters to their own personal tastes, but encouraging players to contribute their own moves also helps the game grow and evolve. Players have the opportunity to master their own moves and learn new moves from one another. The anonymous online format lends itself to experimentation, giving players the opportunity to try things that they might otherwise be too embarrassed to do. In Chapter 4, we describe the technical challenges involved in the development of tools that permit users to select and train their own dance poses.

\subsection{Avatars}

Many games let players select their own character to represent themselves in the game, but representations can reflect real life players to varying degrees. The level of personalization varies widely. The Nintendo Wii allows users to specify details of all facial features, body type, as well as clothing and accessories (see Figure 2.1). The 
flexibility and anonymity of avatars gives players the opportunity to express themselves freely, while still maintaining their privacy. We discuss privacy in more detail toward the end of this chapter.

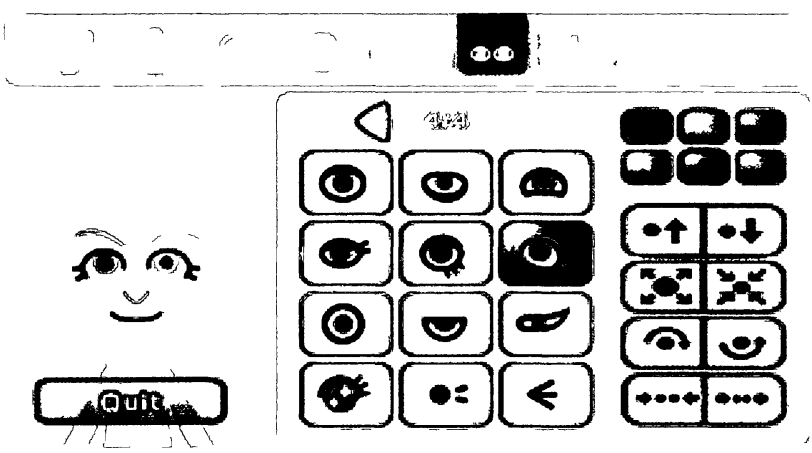

Figure 2.1 Menu for the Nintendo Wii Mii avatar setup

\subsection{Music}

Music plays an important role in games and serves multiple purposes as a tool for communication and personal expression [84]. Music acts as a strong intrinsic motivator, improving mood and providing listeners with a greater sense of empowerment. Motivational music can reduce perception of exertion, increase endurance, improve affect, and increase exercise program adoption and adherence. Music also helps take the focus away from physical exertion and can help players to reach a flow state. [85]. Music tempo can be used as an effective means of controlling player pace in physical activity. Increasing the beats per minute (BPM) of the song can encourage players to move more quickly, while a lower BPM tends to calm players down. Individuals vary widely in their taste in music and, consequently, what they find motivating. There are a number of musical features that impact the effectiveness of a particular song for motivation, including rhythm, musicality, cultural impact, and association, however, these characteristics are not consistent for all people [85]. While it may not be appropriate in all 
games, one way to address the wide variation is to provide players with the tools necessary to select their own music. Participants can then choose songs they enjoy and find motivating.

\subsection{Connection to a Network}

Networked fitness systems can be more persuasive than traditional, non-connected technologies because they can provide more timely and relevant information. Connected devices can gather and report the most recent, and consequently the most persuasive, information [76]. Status information and reminders can provide users with the opportunity to make time for otherwise unplanned activity [53].

Games that are connected by a network are more persuasive because they can provide information that takes into consideration the context, goals, and other variables relevant to the specific user [76]. The system may provide flexibility for contingency by giving users the option to skip through activities and avoid games they cannot play immediately, due to constraints like weather, or number of players required [3]. The more information the system has about individual users and their contexts, the more tailored, and consequently enjoyable, the gaming experience.

\subsection{Novelty}

By allowing players to customize game play and generate their own content, MMOGs maintain constantly renewable sources of novelty and variety. Game developers must invest upfront in the development of tools that allow for customization, but once they release the game, players can effectively take over. Participants will contribute new songs 
and new dances and, as a result, the game world, both interactions and experience, evolves for the benefit of all players. The MMOE provides many avenues for growth and evolution of ideas, for example, through the use of custom avatars and creation or modification of environment elements. The social aspects of the MMOE also contribute to novelty, encouraging players to participate actively in forming new friendships and interacting with people they might not otherwise encounter.

\subsubsection{Social Motivation}

Humans are inherently social creatures and other people play a key role in motivation and persuasion [76]. Whether through exercise classes, training buddies, or even strangers working towards similar fitness goals, social influence can have a huge impact on users' enjoyment and determination. Players may form social alliances and friendships, some of which even extend beyond the game itself, while other players benefit from real-life competitors. In the case of online games, as with any other online social environment, privacy is an important consideration and will be discussed along with the other social factors in the following sections.

\subsubsection{Friendship and Social Facilitation}

Most people exercise more effectively when they are with other people. The principle of social facilitation states, "people are more likely to perform a well-learned target behaviour if they know they are being observed via computing technology, or if they can discern via technology that others are performing the behaviour along with them" [76]. The representation of other participants need not be realistic. Avatars and other abstract 
icons or symbols can simulate social presence. Virtual presence may also produce many of the same beneficial social facilitation effects [76].

Despite the common image of solitary gamers portrayed by the media, video games can provide an opportunity for real-world social interaction. Game players are far from antisocial. They meet with friends outside of school and work even more frequently than non-players and the majority of research suggests that game players are more social on the whole than non-players [79]. People may play alone, but games often invite and encourage social interaction, through both competition and cooperation. Comparable to the collectivist 'team performance' strategy [86], Fogg's principle of cooperation states that "computing technology can motivate users to adopt a target attitude or behaviour by leveraging human beings' natural drive to cooperate" [76]. Cooperation encourages people to work together to achieve shared goals. Video games encourage team-like camaraderie, similar to that found in sports. Players may also play single-player games with a group, competing informally by taking turns and watching each other play [78]. Games give people an opportunity to socialise casually, with many players gathering formally, for example with LAN parties, or informally in common spaces [87]. Social interaction is one of the main reasons young people begin playing video games. Games provide a venue for players to interact with one another and provide insight into one another's personalities [81]. Players may help and learn from one another, talking about the game when they are not playing and improving their strategies. This can help keep players motivated and interested in the game, which is particularly advantageous for exergames, which require regular play. Problem solving and discussion with other 
players can also help people develop social and communication skills, providing players with additional benefits beyond exercise [79].

Connected devices allow information to be sent instantaneously to third parties, including coaches, mentors, and friends. This communication provides motivation through praise, encouragement, or even just passive monitoring. Friends can be an effective source of motivation, encouraging one another to exercise together to reach their goals [51]. Social factors raise awareness, encourage reflection, and increase motivation to lead an active lifestyle. The interaction can also be supported with communication tools like texting or instant messaging [1]. Some persuasive exercise systems let participants choose to share their updates with all, some, one, or none of the other participants. Sharing groups were significantly more likely to meet their goals than individuals were. Participants enjoyed social support and receiving recognition and encouragement from friends [53]. On-going information from friends provides players with a reference point against which to compare their own progress [16]. Some of the participants find motivation in the competitive benchmarking mechanism of watching the progress of each other [4]. Others feel social support from just knowing how their friends are doing. This social comparison also motivates players to exercise more to avoid having the lowest score [53]. Some systems record individual activities, but also encourage players to exercise together and participate in group walks that help everyone. Females in the one study rated group performance as the most powerful method of behaviour change [51]. Group sharing encourages participants to arrange exercise opportunities that would collectively increase 
their fitness levels. Participants of one study found this cooperative approach to be a compelling motivator [51].

MMOGs provide a broader range of interaction possibilities and features that encourage communication, including channels for conversations and questions among groups. Announcements can be made available to one or multiple players at a time. Players can also communicate one-on-one with friends, through an instant-message style list. Interpersonal relationships are integral to the $\mathrm{MMOG}$ experience for most players and the game is setup to encourage social interaction, with relationships developing out of competition and cooperation. In-character relationships often develop within the realm of the game itself; however, out-of-character relationships also develop between players, who may exchange more information outside of the game. This type of relationship may even evolve into other means of communication, such as email, telephone, or face-to-face meetings [83]. In one MMOG study of 5064 respondents, $60 \%$ of participants contacted other players outside of the game, $86 \%$ of those via email, $46 \%$ by phone, and $8 \%$ with paper letters. Of the respondents, 144 players were dating, 25 were engaged, and 12 married partners they met through the game [87]. People are motivated by social interaction in games and exergames can leverage these in-game relationships to encourage players to return and play more frequently.

\subsubsection{Competition}

Computing technology can motivate users to adopt a target attitude or behaviour by leveraging human beings' natural drive to compete [76]. Competition is perhaps the most powerful group-level intrinsic motivator and it is important to overall enjoyment [1]. In 
Western societies, people tend to care more about the outcome when they are competing against others [76]. They are energized and motivated to participate and engage more fully in the activity [7]. Players can also derive satisfaction from competition against themselves. Competition is also a major factor in video game enjoyment; however, players quickly lose interest in a competition where it is easy to cheat [1].

Competition is an extremely important and successful motivating factor for many players. People can use game competition to express aggressive feelings in a safe way and combative behaviour is generally more socially acceptable in the video game context than in real life. Video games elicit an adrenaline rush that is similar to real-world combat. Succeeding in video games helps players feel better and establish dominance in a particular area. Winning games can act as a substitute for real-world success and social acceptance and many players thrive in an environment that lets them prove their skills. Competitive games can also help establish a social ranking and they give winning players an opportunity to brag about their achievements. Some players, especially males, compete for pride and money. The power of competition as a social motivator lies in the reactions of other participants to the winning player and the subsequent establishment of a hierarchy within the group [81]. Opponents can be fictional, real participants who previously played with the system, or a version of the user, using his or her own past game trial. Anecdotal evidence suggests that competition against another player is more enjoyable than playing against a machine [83]. Competition also need not occur among players of comparable ability levels, provided the system makes some accommodation for the difference. Instead of direct competition, measuring who moves faster or burns 
more calories, an intelligent device might have users compete against each other, based on their level of achievement, relative to their own, individual and predefined goals [1].

Exploratory field interviews found that fun and competitive exercise motivates teenagers; however, there was concern that competition might also lead to excessive exercising. Instead of overt competition, the Chick Clique system gives participants information about their friends' activity levels. Individual users may then be more motivated to exercise [51]. It should be noted that players respond differently to competition in games. Some find competitiveness to be an enduring motivator, while some find it unnecessary or even incompatible with the spirit of the game and others feel that competition provided a stimulating benchmarking mechanism [13].

Competition may also help players to leverage the principle of social comparison, which states that "people will have greater motivation to perform a target behaviour if they are given information, via computing technology, about how their performance compares with the performance of others, especially others who are similar to themselves" [76]. The presence of others provides players with a method of benchmarking, allowing them to better monitor their own progress.

\subsubsection{Privacy}

Technology makes it possible for people to interact and receive personalized feedback, without direct observation, from others. Online games can provide a safe and private environment for players to share as much or as little about themselves as they choose. Anonymity and privacy allows players to try new things that they might find difficult or 
embarrassing in public. The game provides the necessary tools, feedback, and guidance for participants to practice and develop their skills. As players improve, their confidence increases and they may then be eager to share their achievements with other players and friends, further increasing their enjoyment, motivation, and self-esteem [79].

Avatar-mediated communication provides varying levels of privacy for game players. Role-playing in MMOGs is typically acceptable, if not widely encouraged. Players communicate through their characters and they choose to disclose as much or as little information about their real lives and selves as they are comfortable sharing. Like other online applications, MMOGs provide greater anonymity as player age, gender, and identity are not identifiable in the virtual game world [88].

Despite layers of protection offered by anonymity, it is important that online games provide players with full control over their personal information and privacy. Players should be free to disclose what they choose, but they should not be required to sacrifice their privacy and anonymity in order to play the game. Players should also remain in control over their contact with other players. Some people may choose to play the game with a small subset of real-life friends. This could be achieved by providing players with the option to exclude interactions from Internet strangers or limit interaction with individual user block and allow lists.

Privacy poses a unique challenge for an MMOE that allows players to select their own, full-body poses. Dance poses require accurate representations in order to effectively 
communicate the specifics to other players. However, many players may be hesitant to share full body photographs of themselves with strangers, or even friends. There are several image processing strategies that may reduce or even eliminate these concerns, by blurring or otherwise disguising the players' identity. These issues are discussed further in section 4.2 .

\subsection{Massively Multiplayer Online Games}

A massively multiplayer online game (MMOG) is a video game that allows hundreds, or even thousands, of participants to play with and against one another in a persistent, virtual world or environment that they can explore. Thousands of people can play simultaneously, exploring and interacting with the same virtual environment. Both the world and the characters are persistent, essentially forming a parallel reality. MMOGs consequently support a great deal of social interaction, and by allowing users to generate their own content, player experiences and the game itself continue to evolve. In this section, we discuss the history, popularity, and demographics of MMOGs, different types of MMOGs, and specifics of virtual game worlds, avatars, and content.

\subsubsection{MMOG History, Popularity, and Player Demographics}

People play MMOGs online through personal computers or newer gaming systems that provide access to the Internet, for example the Nintendo Wii, Xbox 360, PSP, and PlayStation 3. MMOGs evolved from text-based role-playing games called MUDs, short for multi-user dungeons/domains, which were common in the late 1990s. Most MUDs would support around a hundred players at any given time, but rarely utilized that capacity. The business market for online gaming and MMOGs is growing rapidly. The 
most popular MMOG is Blizzard Entertainment's World of Warcraft (WoW), with more than 3.5 million global customers in 2005 [83]. NCSoft's Lineage peaked at 4 million subscribers in 2005. However, while WoW has proven hugely successful, surveys of MMOG participants indicate that players are predominantly young adult males. Males represents approximately $85 \%$ of the EverQuest population and as much as $89 \%$ of the combined demographic for EverQuest, Dark Ages of Camelot, Asheron's Call, and Anarchy Online. In contrast, The Sims Online has a majority female population, accounting for approximately $60 \%$ of players [83].

\subsubsection{MMOG Genres}

Many of the most popular massively multiplayer games are from the role-playing game (RPG) genre, including WoW, EverQuest, and Lineage, but MMOGs exist for a number of different genres, including action, building, exploration, flight simulation, first-person shooter (FPS), real-time strategy (RTS), and social games. Some popular, social MMOGs include Second Life [89], Toontown [90], and The Sims Online [91]. There is even an MMOG dancing game, Linden Research's Dance! Online, which is based on Dance Dance Revolution [92].

\subsubsection{Virtual Game Worlds, Avatars, and Content}

Virtual MMOG worlds, though digital and intangible, represent a real physical environment. While additional supernatural creatures, powers, and characteristics may supplement the environment, the majority of the world characteristics mimic our own. The persistent game world should, at least in theory, exist and be available to players all day, every day. One or more computer servers house the virtual world and users access 
the game from all over the world. Persistence allows communities, economies, reputations, and social structures to emerge and evolve. As a result, it is easy for players to invest and involve themselves in the game. Persistence in MMOGs is dynamic, meaning that even when the player is offline and not playing, the virtual world continues to change, through the presence and actions of other players. Players cannot control the events in the world by withdrawing, for example, from a battle by turning off the computer. In fact, there are limited opportunities for natural breaks or pauses. This, combined with an established norm that players not abandon a group mid-combat or in the middle of an excursion, can make for a highly addictive environment [83]. Avatars are also persistent, enabling people to maintain a consistent character persona within the game world. Players typically choose personality and physical characteristics of their avatars. Representations may reflect real-life people, or they might also be fictitious. Identity persistence permits social interaction and the development of interpersonal relationships [83].

In the virtual world of an online game, players can generate new content on an on-going basis. It provides an opportunity for players to shape and contribute to their own gaming experience. Creative participation and active involvement can increase players' interest and investment in the game. Moreover, user-contributed content allows the MMOG to evolve to suit the needs of its players. Game developers need not create additional content as players have the freedom and flexibility to create new levels and challenges themselves. User contribution and the evolving nature of MMOGs helps provide players with a continually novel experience, keeping players engaged over a longer term. The 
virtual worlds of MMOGs may also provide positive environments for health-related applications. Future exergame systems could leverage the social and motivational aspects of MMOGs to provide an alternative to traditional health care treatment and prevention methods [36].

\subsection{The Sensor Network for Active Play (SNAP) System}

The Sensor Network for Active Play (SNAP) system is a wearable sensor network that uses body position as input for video game applications. Tri-axis accelerometers, placed on players' wrists and above their knees, infer full-body poses. Several games have been developed for the SNAP system, including Posemania and Dance Fighter, in which players replicate full-body dance poses to music [11]. This chapter is a summary of earlier results, providing a foundation for the later design of a massively multiplayer online exergame. The SNAP system offers a practical solution for exergame input.

\subsubsection{Introduction to SNAP}

We developed the SNAP system, inspired primarily by the novelty introduced by Nintendo Wii and our displeasure with issues using the system. The first is what we call the lazy-Wii phenomenon. Once players realize that a quick flick of the wrist will suffice rather than a full and complete tennis swing, it becomes apparent that the system is not as sophisticated as players initially thought, and they tend to return to a less active form of play. The second is the issue of incorrect form: proper form and technique are not significant factors in game play. Regardless of additional sensors (for example, the Wii Fit balance board), the system cannot determine whether the person is performing yoga poses in the right way or if the participant is doing exercises correctly. 
Accelerometers have inherent limitations when it comes to detecting motion. Tri-axis accelerometers supply only three pieces of information (X, Y, and Z acceleration data), so it is impossible to know the exact rotation and position in space. In this respect, an accelerometer cannot act as a replacement for a gyroscope and cannot accurately detect the amount of movement itself. Despite these limitations, it is still possible to recognize and detect full body poses using several sensors, at a sufficient rate to allow a great many applications.

We believe that the next logical progression in exergame system development is a multisensor network, with sensors placed on strategic areas of the body to allow for an even more immersive gaming experience. Such a network allows users to replicate elaborate dances, yoga and tai chi poses, and can verify the accuracy of each pose. Although it is desirable to have a system that easily interprets human input, with practice users can develop and perfect the application-specific skills. Four sensors are sufficient to enforce form and require the player to engage in designed activities [10]; however, for training applications such as yoga, Pilates and tai chi, a larger sensor network is preferable as the goal is to improve form and train rather than provide fun and entertainment. The more sensors in the network, the more accurately we can define the body pose and motion [10].

\subsubsection{Design and Implementation of the Sensor Network}

In this section, we describe the design and implementation of our sensor network and the software components that make up the recognition system. 


\subsubsection{Sensor Pods}

Our sensor network consists of a variable number of sensor pods depending on the application in question. We have found that four sensors are suitable for entertainment related applications, while eight to sixteen better serve training applications [10]. Each pod contains two layers of foam that sandwich a $2 \mathrm{~g}$ tri-axis accelerometer, ensuring that the sensor remains secure in its position and minimizing unwanted movement on the sensor itself. The casing also protects the chip and connection from motion based wear and tear. The sensor pods are illustrated in Figure 2.2.

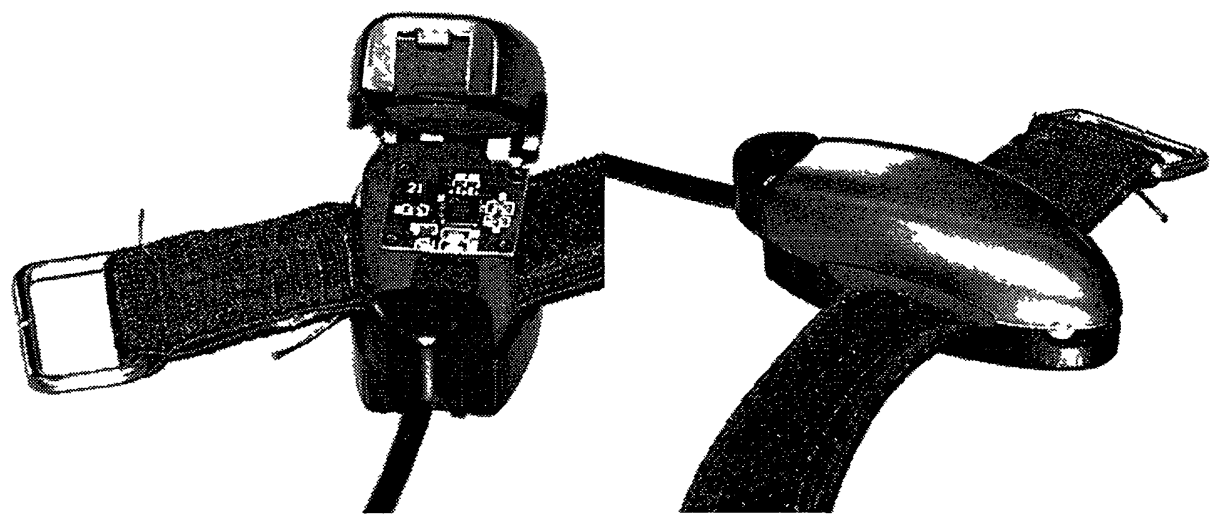

Figure 2.2 Plastic pods used to encase accelerometers with straps

\subsubsection{Sensor Pod Placement}

The nature of the poses and the application dictate the placement of accelerometers on the body. A truly generic system would be comprised of sensors on all of the major bone segments in the body. However, this is not necessary for all applications, for instance if orientation information for specific parts of the body is not required. In our example application, it is necessary to get position and rotation information of both arms and legs. As illustrated in Figure 2.3, we place sensors on the wrists and above the knees, to minimize movement, slippage, and excessive rotation once mounted. The system does 
not require precise placement of the pods, and each application has the freedom to place pods where appropriate given the aforementioned constraint. However, the sensor pods placement must be consistently in the same general vicinity and orientation on each new or returning participant.

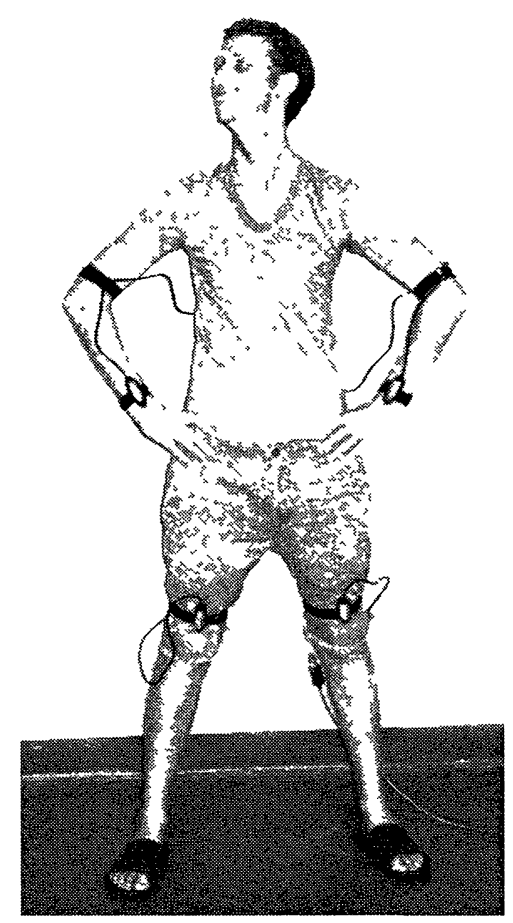

Figure 2.3 The placement of the 4 sensor pods

\subsubsection{Pose Detection}

As with any classification technique, we rely on basic training and recognition (game playing) phases from classical pattern recognition theory [93]. This makes the sensor network reusable for many different contexts as body position and orientation information are easily gathered and the recognition system trained by example. We use the rotation sensing capabilities of the accelerometers and their placements on the body to determine poses that players re-create. We use this pose recognition system to create a 
number of dancing oriented games that require players to replicate dance poses in time with the music. We briefly discuss the training and recognition phases next.

\subsection{Training Phase}

The purpose of the Training Phase is to create a data set from a given number of example poses by an individual. A reading consists of the $\mathrm{X}, \mathrm{Y}$, and $\mathrm{Z}$ acceleration data for each pod in the sensor network. We take readings from a number of people to create the entire data set, thus determining a range of acceptable positions. To record a pose, the subject performs each pose five times. Informal testing led us to take 500 readings each time, over a two-second period, with a break between; yielding 2500 separate readings. We have found that good, real-world recognition rates occur when the sample size consists of at least five people and they are of diverse body types. More samples in the training data set allows more variability to be accepted in the recognition phase. Thus, for entertainment applications, a larger data set is encouraged. However, for training applications, where the goal is to learn the proper body positions of an expert or trainer, a smaller training data set is sufficient, perhaps even more desirable.

We treat the readings as points in $\mathrm{N}$-dimensional space and compute the centroid and convex hull of the volume created by all of the sample points. We compile the data for each axis of each accelerometer for each pose and store it in a pose bank, for use in subsequent recognition tasks. A pose bank is a collection of information from each of the sensors attached to the body for all defined poses. 


\subsection{Recognition Phase}

As a game or application runs, data from the sensor network pods are read in continuously and compared to the poses stored in the pose bank. We informally tested several alternate equations [11], but a minimum mean distance rule classifier (outlined in Equation 1) [93] proved to be most successful for the recognition system. It characterizes each category (pose) by mean and standard deviations of the components of its training data. The distance, $d(M, m)$, between an unknown sample $M$ (input move) and the mean of the features of class $m$ (trained pose), is then computed. The unknown sample is assigned to class $\mathrm{m}^{*}$ (recognized as move $\mathrm{m}^{*}$ ), for which such distance $d(M, m)$ is minimum for all $\mathrm{C}$ classes. Formally:

$$
\mathrm{m}^{*}=\operatorname{Min} \mathrm{d}\left(\mathrm{M}, \mathrm{m}_{1}\right)\{\mathrm{i}=1,2 \ldots \mathrm{C}\}
$$

$\mathrm{d}\left(\mathrm{M}, \mathrm{m}_{1}\right)$ is the Mahalanobis distance metric, outlined in Equation 2. Introduced by P. C. Mahalanobis in 1936 [94], it is based on correlations between variables by which different patterns can be identified and analysed. It is a useful way of determining similarity of an unknown sample set to a known one. It differs from Euclidean distance in that it takes into account the correlations of the data set and is scale-invariant. If the covariance matrix is diagonal, the resulting distance measure is the normalized Euclidean distance:

$$
d(\vec{x}, \vec{y})=\sqrt{\sum_{i=1}^{N} \frac{\left(x_{l}-y_{2}\right)^{2}}{\sigma_{2}^{2}}}
$$

where $\sigma_{l}$ is the standard deviation of the training data $\left(x_{l}\right)$ over the sample set. The Mahalanobis distance is the distance of the test point from the centroid, divided by the width of the convex hull in the direction of the test point [94]. If the distance falls within 
an acceptable threshold for a known pose, the pose is deemed detected. When checked against all poses in a pose bank, the minimum distance classifies the pose.

\subsubsection{Experimental Results with SNAP}

We conducted a series of preliminary experiments to establish the capabilities and limitations of our sensor network, specifically to determine the feasibility of applying similar principles in a video game context [10]. A summary of the results are included here. Participants performed multiple trials, replicating eight distinct poses. For these experiments, we selected poses that ensured reasonable differences in limb orientation. We conducted tests in a supervised lab setting, with a consistent level of instruction and coaching provided by the trainer, to examine the impact of varying amounts of training data, the size of the sensor network, practice, pose difficulty, and the process of determining appropriate, application-specific recognition thresholds. The eight poses are illustrated in Figure 2.4. 


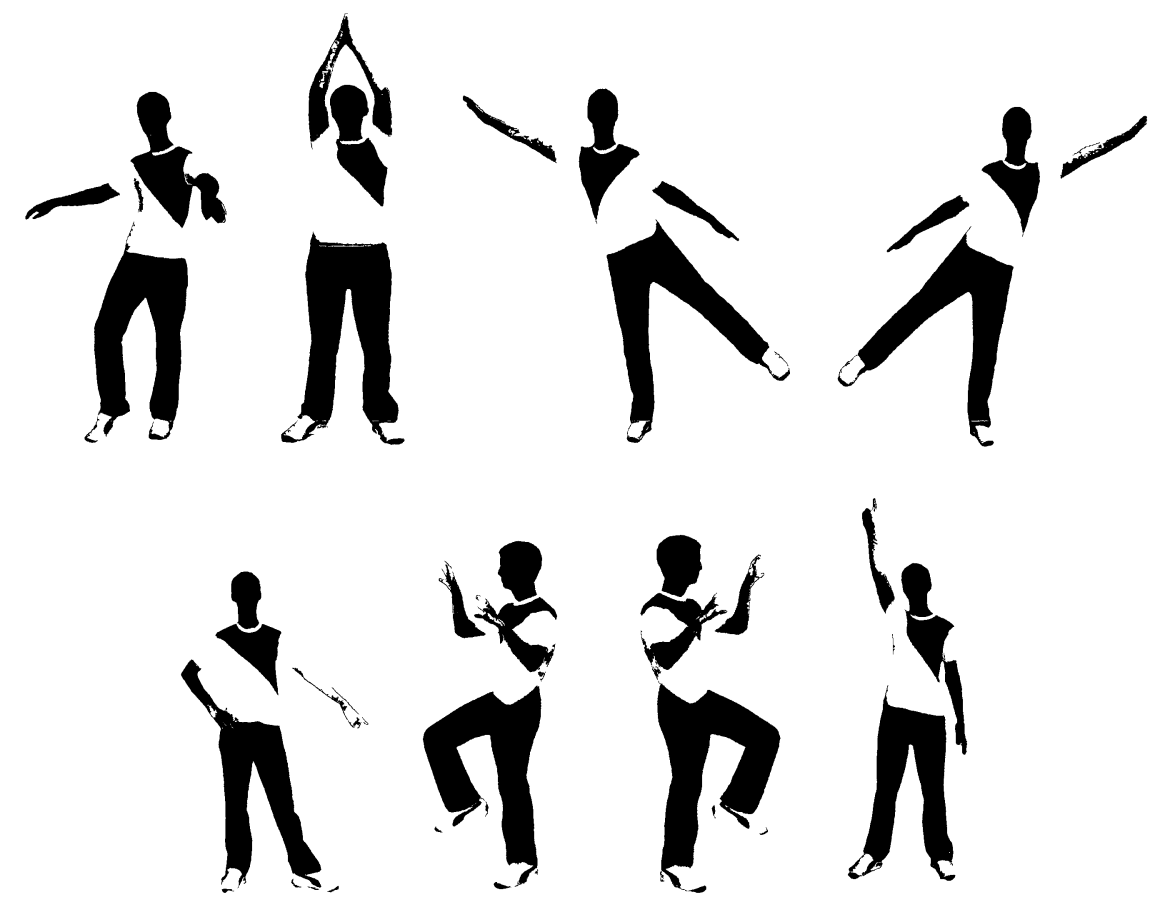

Figure 2.4 The eight poses, inspired by Michael Jackson's Thriller

It is unreasonable to expect a user to be able to achieve a perfect pose, with a recognition distance of zero, even if he or she is a part of the training sample. Simple variations are inevitable due to core mechanics of the human musculature system and the variability in the placement of the sensors on the body. Our experiments test how well a person can play against their own training data compared to another person's training data. This experiment has significant impact on the practicality of the system. Participants were given a pose to replicate in a minimum amount of time. Once the pose was called for, the system continuously searches for the pose in a prescribed amount of time, typically less than a few seconds. The overall recognition rate for all trials and all poses is $74 \%$ when testing against training data set created by a different individual. This is not sufficient for a truly interactive application. However, with self-trained data, the recognition rate is $100 \%$, meaning that within the context of these experiments, users were consistently able 
to replicate the poses that they trained. This is fundamentally important as game developers can ensure that the game is playable by everyone, regardless of body shape or physical limitations. Developers need only incorporate a training phase, already common in many games, into their game.

Through the experiment, it became apparent that even with practice, some participants are unable to replicate certain poses trained by another individual. Physical limitations make it impossible for some subjects to get their bodies into the exact same position as the single person who provided the training data. These problems arise from variations in muscle size and volume, limb length, proportions, and overall body type. Combining training data from multiple people makes it possible for a wider variety of users to achieve better recognition rates. To test this, we collected training data from seven individuals of varying heights and shapes. We combined the seven sets and creating a generalized pose bank with the larger training sample. This allows greater variation in the range of acceptable poses and makes the system generally more usable by people who are not a part of the training data set. Variations of a few degrees in the limb positions are generally acceptable, but the application dictates how much variation is acceptable. This phenomenon results more from the training process accounting for differences in body sizes, shapes and flexibilities than a deficiency in classification methodology and supports our assertion that the stricter the pose and position needs to be, for example as in a training application, the smaller the amount of training data necessary. 
Confusion matrices, as shown in the tables below, are used to determine the thresholds values and reduce the likelihood of false positives among other poses, while maximizing the likelihood of true positives.

\begin{tabular}{|ccc|ccc|cc|c|c} 
Pose & $\mathbf{1}$ & $\mathbf{2}$ & $\mathbf{3}$ & $\mathbf{4}$ & $\mathbf{5}$ & $\mathbf{6}$ & $\mathbf{7}$ & $\boldsymbol{8}$ \\
$\mathbf{1}$ & $\mathbf{3 . 9 9 6}$ & 16.386 & 12.308 & 12.074 & 20.178 & 25.487 & 29.183 & 38.874 \\
$\mathbf{2}$ & 15.878 & $\mathbf{3 . 1 0 4}$ & 14.394 & 22.878 & 33.016 & 33.768 & 20.393 & 53.646 \\
\hline $\mathbf{3}$ & 15.993 & 27.239 & $\mathbf{2 . 1 9 2}$ & 16.053 & 22.355 & 44.357 & 34.821 & 18.212 \\
\hline $\mathbf{4}$ & 10.918 & 32.047 & 16.059 & $\mathbf{2 . 8 6 8}$ & 16.341 & 45.845 & 39.553 & 43.530 \\
$\mathbf{5}$ & 9.704 & 37.074 & 12.302 & 11.682 & $\mathbf{3 . 7 7 3}$ & 48.108 & 47.171 & 25.971 \\
$\mathbf{6}$ & 22.948 & 21.116 & 27.062 & 23.684 & 45.247 & $\mathbf{3 . 3 1 5}$ & 26.668 & 56.591 \\
$\mathbf{7}$ & 43.606 & 27.261 & 16.094 & 30.934 & 43.458 & 35.509 & $\mathbf{3 . 2 1 2}$ & 60.027 \\
\hline $\boldsymbol{8}$ & 12.896 & 28.030 & 8.702 & $\mathbf{1 8 . 9 7 0}$ & 29.038 & 39.525 & 36.946 & $\mathbf{3 . 9 9 2}$ \\
\hline
\end{tabular}

The results indicate little possibility for false positives. The average multiplying factor for a correct classification versus a misclassification is actually higher for multiple training data sets, meaning that the likelihood of false positives goes down as the amount of training data goes up. Table 2.2 shows the multiplying factors for each pose. It indicates that the difference between a correct classification and a misclassification is proportionally increasing, and thus there is significant value in a larger training set.

\begin{tabular}{|c|c|c|c|c|c|c|c|c|c|}
\hline & \multicolumn{10}{|c|}{ Pose } \\
\hline \multirow{2}{*}{$\begin{array}{c}\text { Training } \\
\text { Set Size }\end{array}$} & & $\mathbf{1}$ & $\mathbf{2}$ & $\mathbf{3}$ & $\mathbf{4}$ & $\mathbf{5}$ & $\mathbf{6}$ & $\mathbf{7}$ & $\boldsymbol{8}$ \\
\cline { 2 - 10 } & $\mathbf{7}$ & 3.44 & 4.90 & 3.24 & 3.46 & 3.91 & 4.46 & 5.29 & 6.47 \\
\hline
\end{tabular}

Table 2.2 Average pose multiplier factors for single and multiple training data set sizes

As Table 2.3 exhibits, the recognition rates increase substantially as the size of the training set increases. For entertainment applications, where recognition rates must be higher for the general population, more training data is preferable.

\begin{tabular}{|c|c|}
\hline Number of Data Sets & Successful Recognition (\%) \\
\hline 1 & 37.5 \\
\hline 7 & 87.5 \\
\hline
\end{tabular}

Table 2.3 Effect of multiple training data samples on recognition rates 


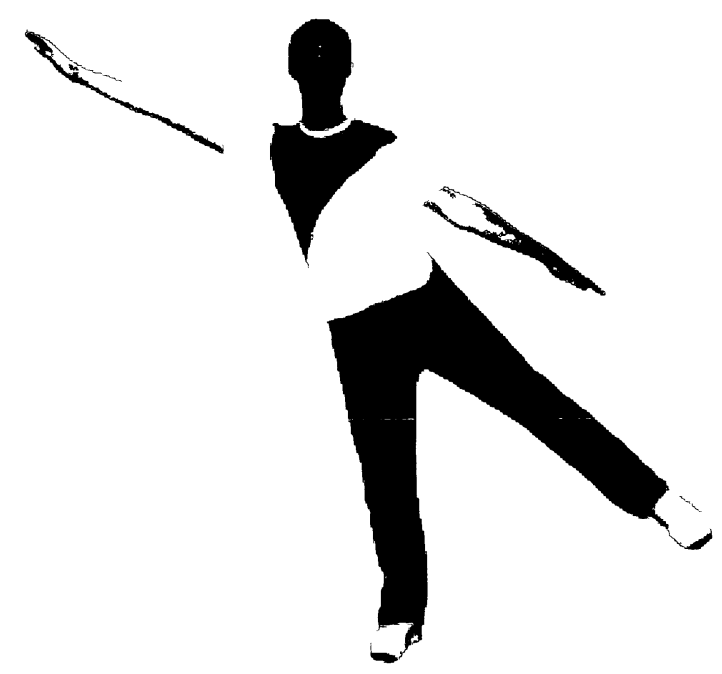

Figure 2.5 Pose 3 used in experiments

The next experiment independently validates the suggestion of Bao et. al [95] that the more accelerometers there are in a sensor network, the more accurately activities can be differentiated. Table 2.4 shows that increasing the number of sensors makes more demands on a single user to properly replicate the pose, shown in Figure 2.5, since each added sensor contributes to the overall distance from the optimal pose. The higher average distance per sensor reflects this finding. In game development, we worked with a network of only four sensors, as extremely rigorous pose replication was not the goal.

\begin{tabular}{|c|c|c|}
\hline $\begin{array}{c}\text { Number of } \\
\text { Accelerometers }\end{array}$ & $\begin{array}{c}\text { Mahalanobis } \\
\text { Distance }\end{array}$ & $\begin{array}{c}\text { Average Distance } \\
\text { Per Sensor }\end{array}$ \\
\hline 4 & 2.676948 & 0.669236 \\
\hline 8 & 7.269316 & 0.9086645 \\
\hline
\end{tabular}

Table 2.4 Mahalanobis distances, with networks of four and eight sensors

The effect of practice plays an important role in pose recognition. In our experiments, the error rate drops as the player acquires more experience playing the game. Player improvement is typically most significant after the first few trials. This is an expected side effect of practice and, in fact, should be a desirable outcome from a game design 
point of view. As indicated in Figure 2.6, success rates improve as the number of trials increases.

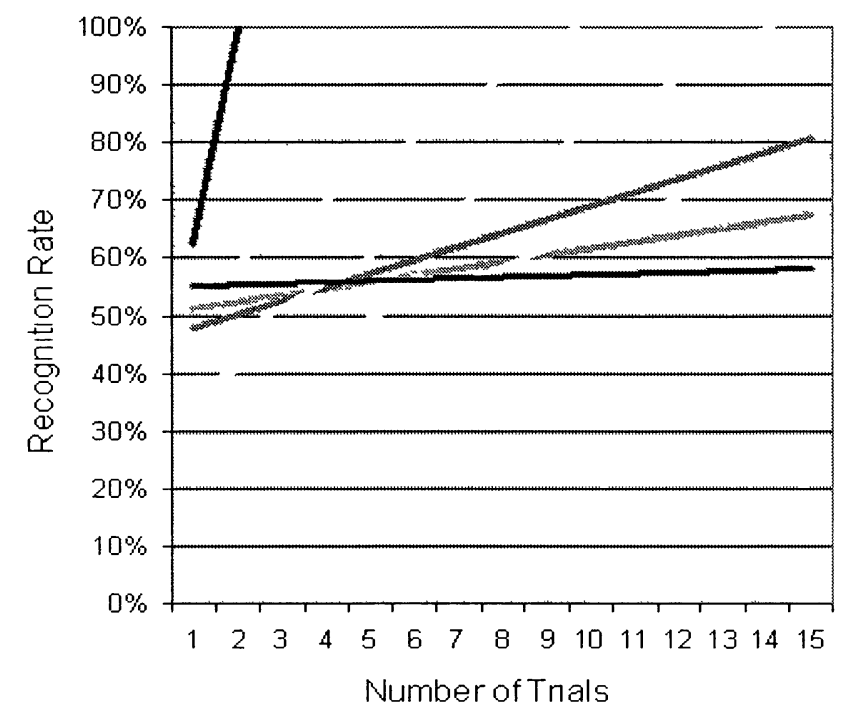

Figure 2.6 Trend lines for the learning curves of seven subjects

We conducted an experiment to examine the effect of repetition on the successful recognition rates. Our hypothesis was that with practice, any user would be able to achieve correct recognition of every pose in a trial. In a video game context, we also want the players to succeed with practice. Seven participants performed the eight poses set out by the pose bank illustrated in Figure 2.4 sequentially, up to fifteen trials, stopping if they successfully executed all poses in one trial. It is important to note that this experiment used a training sample of one, the most difficult and closest to a training scenario. The resulting trend lines, illustrated in Figure 2.6, support our hypothesis in that, given enough repetition; any user can improve their ability to match the orientation and position of a single expert. This validates our claim that the sensor network is useful for training applications where body position and orientation are fundamentally important, such as 
yoga and Pilates. Although there was observed improvement overall, certain poses were problematic for most subjects, which led to further pose-based analysis discussed next.

Clearly different players will learn at different rates, but within 15 trials most players are able to bring their recognition rates up by more than $30 \%$. When we have a larger training data set, the initial recognition rates are higher and all of the people were able to achieve perfect recognition rates with practice. These recognition rates are suitable to allow the game to be trained in the development studio by multiple people and subsequently played by most players without the need for a training phase being built into the game. Moreover, this allows game designers to integrate progressively increasing levels of difficulty with the full knowledge that players can improve with practice.

In a separate experiment, prior to commencing the actual play test, 15 new participants were shown the eight poses and given instruction on how to accurately perform them. Despite moderate levels of continued exertion (and consequently fatigue), 13 of the 15 participants continued to improve when playing for an extended duration of 14 minutes in their final trial. The average improvement was approximately $13 \%$ [13].

\subsubsection{Optimal Number of Data Samples to Include in Training}

Training data is recorded so that it can be compared to 27 different pose banks, containing between one and nine subjects' pose training data. Each of the three trials includes the same training data, with each data set added to the bank in a different randomized order. As such, the trials converge when the training data from all nine participants has been added to the bank. Figure 2.7 illustrates the confusion matrices' 
decreasing average threshold values. The asymptotic nature of the curves suggest that the average threshold distance levels off around five and the inclusion of additional training data will yield diminishing returns.

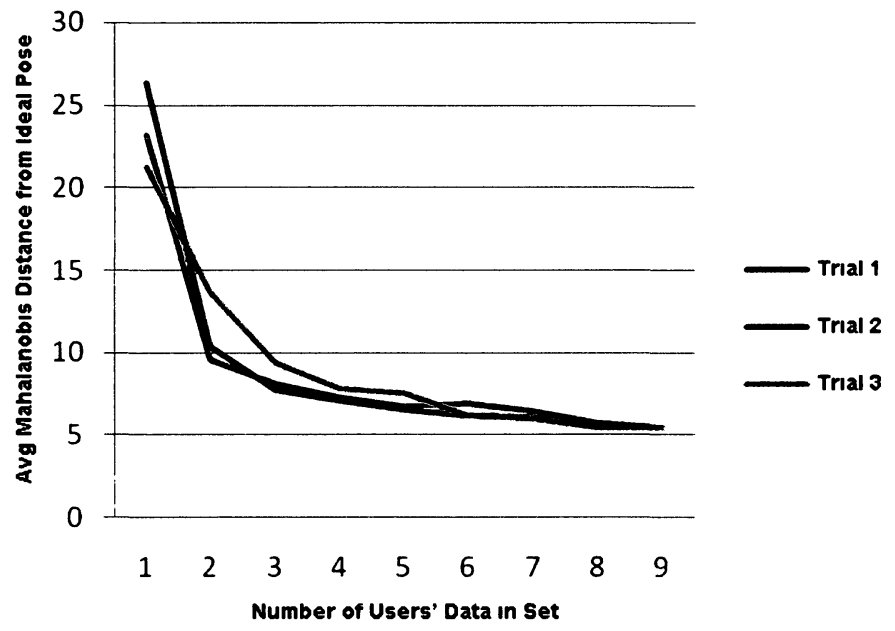

Figure 2.7 Convergence of distances as the training data set increases

\subsubsection{Strictness in Training Poses}

Two alternate methods were used to train pose data. In the first method, subjects replicated the poses seen on screen as they would naturally, and they were recorded this way. Only poses that were clearly misunderstood or incorrect were excluded from the data set. A second series of training data was recorded with stricter enforcement of the ideal pose. Prior to recording, subjects performing the poses were required to adjust their limb positions until they were deemed comparable to the ideal pose by a human evaluator. When playing against the strict training process, users encountered significantly more difficulty in successfully replicating the poses. In some cases, participants were unable to get into the poses at all, regardless of the amount of coaching or limb adjustment. For these reasons, a more natural pose training system is generally 
desirable within the gaming context; however, a stricter system may be desirable for training or simulation applications.

\subsubsection{Technical Limitations of Speed}

In our application, poses are presented at a variable rate. In our trials, the speed was increased to the point where participants were no longer able to replicate the majority of poses. Using a consistent pose validation threshold, one expert player achieved a success rate of $100 \%$ at the speed of 80 poses per minute (PPM). This player was capable of achieving success rates above $80 \%$ at 96 PPM, but Figure 2.8 illustrates the significant drop in success rates between 96 PPM and 120 PPM for an expert player. With practice, it is reasonable to assume that subjects will improve, however it is unlikely that they will reach perfection at a speed greater than 96 PPM. The maximum speed is less a function of hardware limitations and more of human limitations. Settle time for the accelerometers we use is approximately $180 \mathrm{~ms}$. With reaction time accounting for approximately 200 $\mathrm{ms}$, at a speed of $120 \mathrm{PPM}$, (or 1 pose per $500 \mathrm{~ms}$ ), just over $100 \mathrm{~ms}$ remains for participants to move their bodies into the correct position [11].

In trials where the time between poses was less than $625 \mathrm{~ms}$, the expert player would no longer attempt all poses. While the behaviour of skipping difficult poses is not uncommon among new players, it is uncharacteristic for the experts and in this case is a function of strategy as opposed to laziness. With practice at the $500 \mathrm{~ms}$ speed, it became evident to the player that it was not possible to get into pose fast enough so he strategically chose sequential poses that were easier to replicate, skipping a more difficult 
pose in between to prepare for the next. This allowed the player to optimize his results, when reaction and settle times made $100 \%$ success impossible.

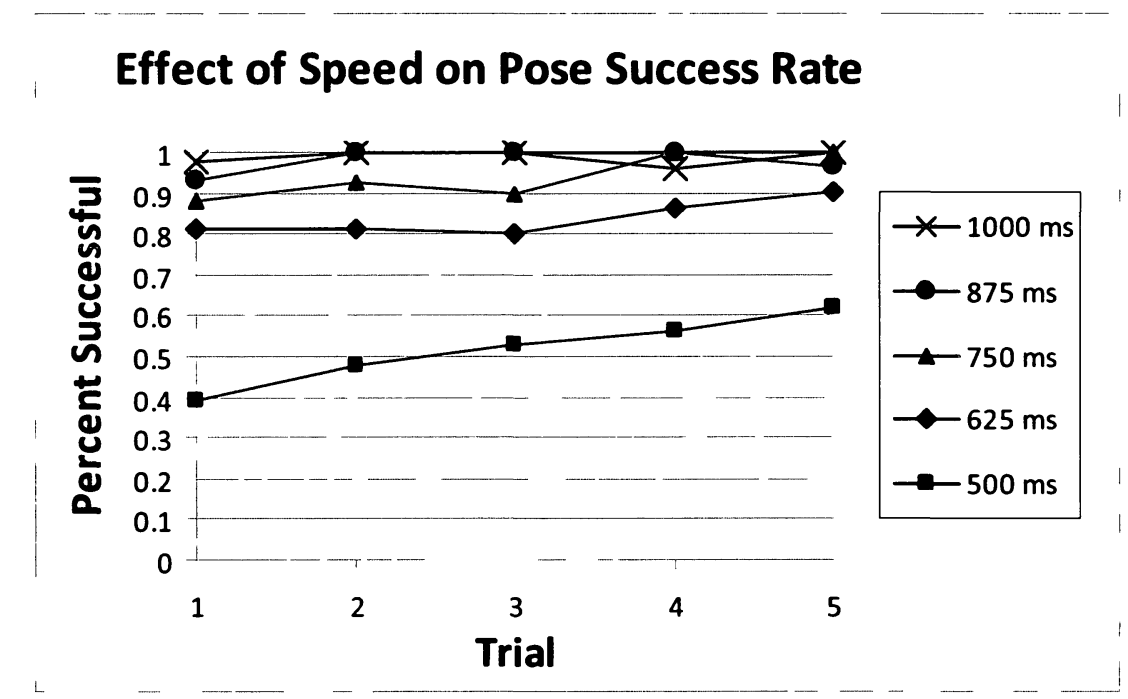

Figure 2.8 Effect of speed on success rate for a single person

It should also be noted that success rates may be superficially increased at faster speeds by using a larger acceptable validation threshold value. The downside of this approach is that it permits players to perform less accurate poses, potentially reducing intended benefits and enjoyment.

\subsubsection{Quantitative Exercise Benefits}

In a separate experiment, SNAP was compared with the Nintendo Wii Step and a sedentary video game (SVG) [13]. Fourteen moderately inactive university aged participants (seven female, seven male) completed the entire study. The additional inclusion criteria were: aged 18-25, not a current sports participant, and not currently exercising vigorously two or more hours per week. Anthropometric data of the participants is shown in Table 2.5. According to their BMI scores, six of the participants were classified as overweight and eight had ideal weights. 


\begin{tabular}{|c|c|c|c|c|}
\hline & Age (years) & Height $(\mathbf{c m})$ & Weight $(\mathbf{k g})$ & Body Mass Index \\
\hline Male & $21.7 \pm 1.8$ & $178.4 \pm 9.8$ & $70.5 \pm 8.6$ & $21.8 \pm 1.9$ \\
\hline Female & $21.9 \pm 1.1$ & $160.5 \pm 2.8$ & $64.0 \pm 9.9$ & $21.9 \pm 1.1$ \\
\hline Total & $21.8 \pm 1.5$ & $170.6 \pm 11.0$ & $67.7 \pm 9.4$ & $23.3 \pm 3.1$ \\
\hline
\end{tabular}

Table 2.5 Participant Stats: Age, Height, Weight and BMI

The Posemania game for the SNAP system has been played perfectly at 80 PPM and is playable at speeds of 90 PPM. For this study, a moderate pace of 60 PPM was used, as players were beginners. During SNAP sessions, participants were first given a demonstration and verbal instructions on how to perform the eight dance poses. The training session was initiated at a speed of 20 poses per minute (PPM) and speed was then increased to 30 PPM. An additional 10 minutes of practice was completed at a speed of 60 PPM (the speed at which they were tested). In all, participants were given 30 minutes of experience with SNAP prior to the official tests. In testing sessions, participants played continuously for 15 minutes, were given a one minute rest, followed by another 14 minutes of play.

SNAP compares well to commercial systems and we expect that better results can be achieved with higher pose rates. The SNAP intervention elicited significantly higher mean exercise heart rates, $117.9 \mathrm{bpm} \pm 16.0$, than both the Wii Step, $99.6 \mathrm{bpm} \pm 12.7$ $(p<0.01)$, and SVG, $74.1 \pm 7.72(p<0.01)$ as seen in Figure 2.9. Mean rates of perceived exertion (RPE) values among and between the three interventions were significantly different $(\mathrm{p}=<0.001)$. Posemania provides enough intensity to elicit weight management benefits whereas the Wii Step did not. 


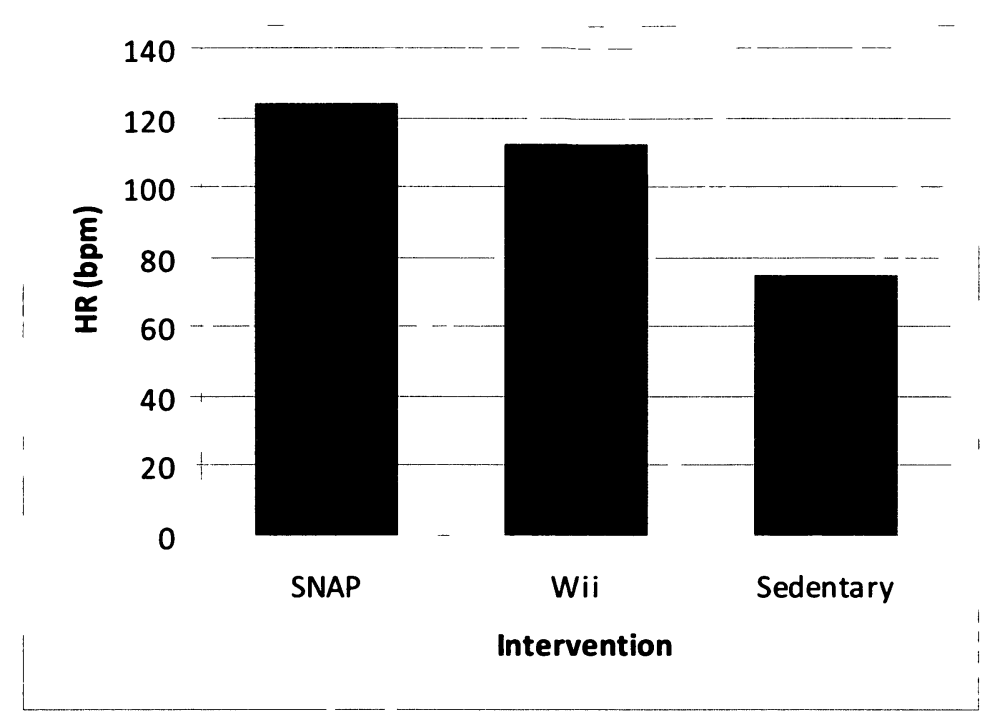

Figure 2.9 Mean heart rate for each intervention

Mean energy expenditure (EE) was calculated for each intervention (see Figure 2.10) using the submaximal exercise predictive equation (Equation 3) [70]:

$$
\begin{gathered}
\mathrm{EE}=\text { gender } *(-55.0969+0.6309 * \mathrm{HR}+0.1988 * \text { weight }+0.2071 * \text { age })+ \\
(1-\text { gender }) *(-20.4022+0.4472 * \mathrm{HR}-0.1263 * \text { weight }+0.074 * \text { age })
\end{gathered}
$$
where gender $=1$ for males and 0 for females.

SNAP was found to be significantly higher intensity exercise than the Wii Step and sedentary video games. 


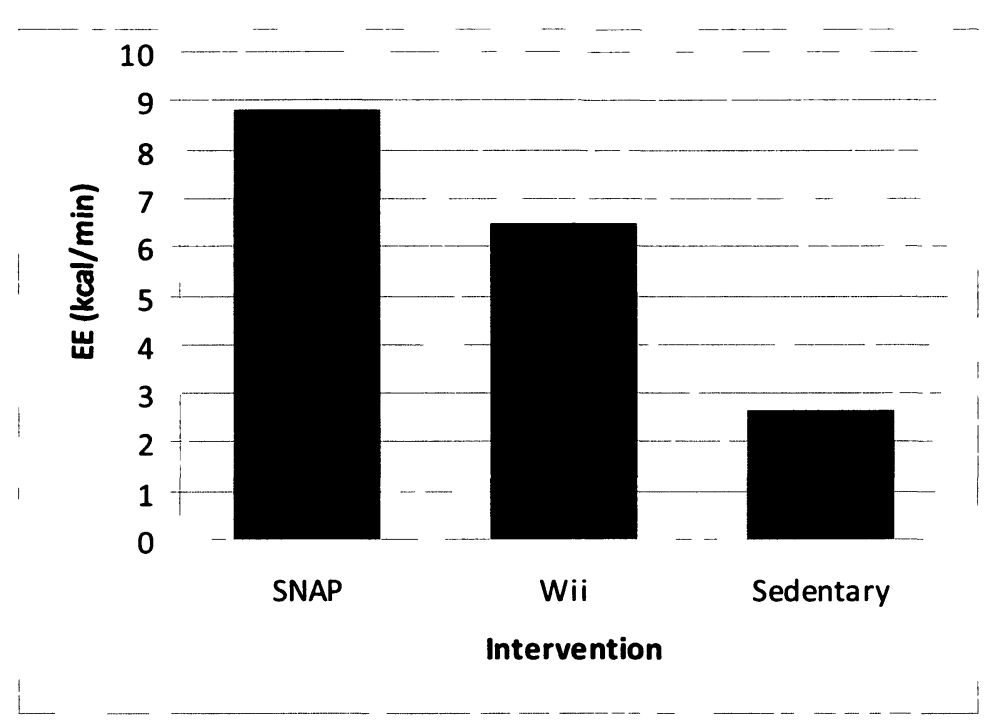

Figure 2.10 Mean energy expenditure for each intervention

The mean EE for the SNAP intervention was $7.3 \pm 1.5 \mathrm{kcal} / \mathrm{min}$. For the Wii Step and sedentary interventions, the EEs were $5.1 \pm 1.4$ and $2.0 \pm 0.65 \mathrm{kcal} / \mathrm{min}$ respectively. There was a significant difference in EE among the interventions $(p<0.001)$. SNAP EE was significantly greater than both the Wii Step and sedentary interventions $(p<0.001)$. The Wii Step EE was also significantly higher than the SVG $(p<0.001)$. The EE of the SVG was significantly less than both the SNAP and Wii Step (both $p<0.001$ ) [13].

\subsubsection{Pose Success and Physical Exertion}

Skill scores were calculated for each participant for the SNAP system as a percent of total poses performed correctly. These scores were positively correlated with the HR of the participant $(r=0.438)$ as shown in Figure 2.11. 


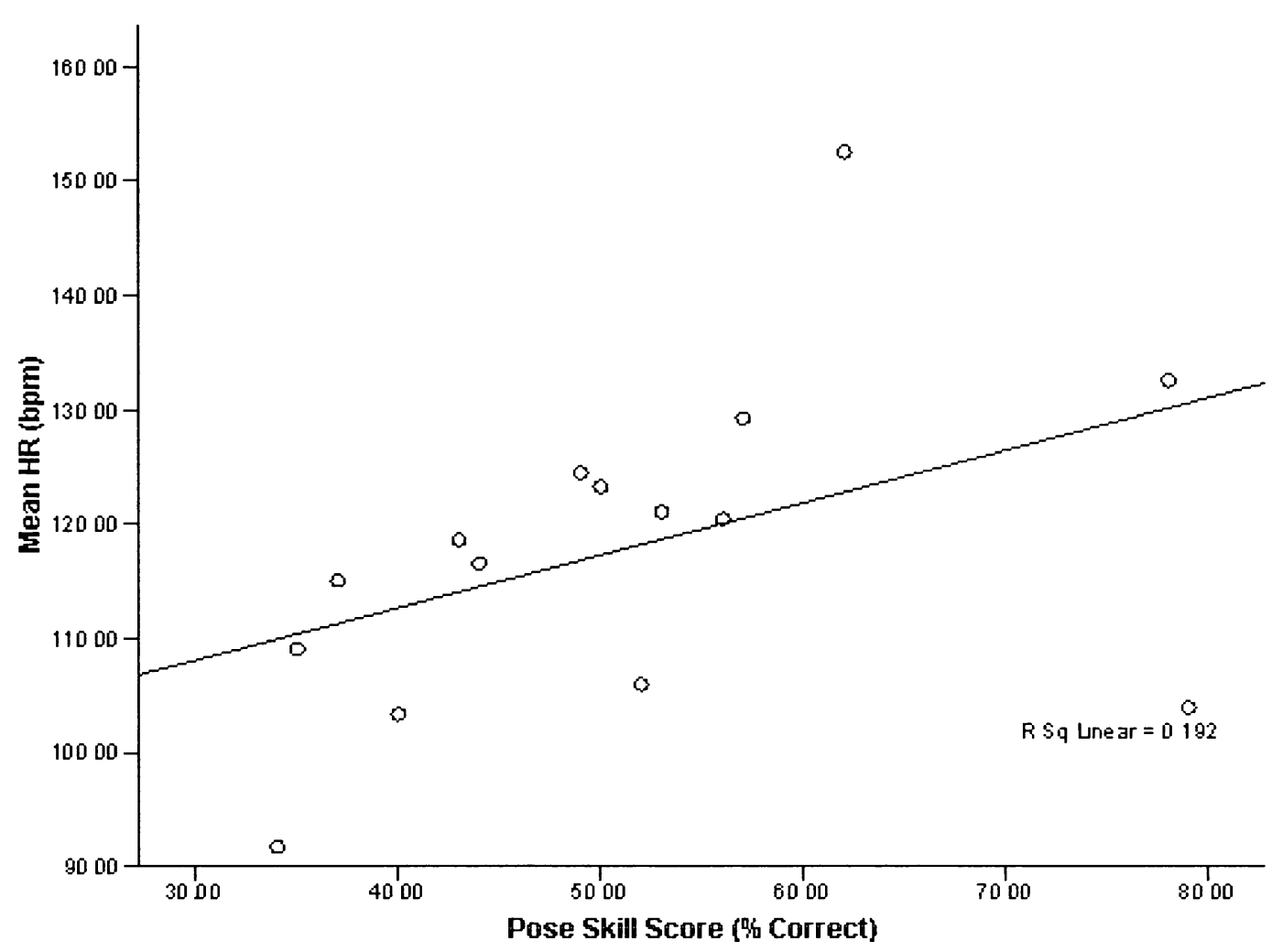

Figure 2.11 Heart rate increases as the ability to successfully duplıcate poses increases

There appears to be a strong correlation between SNAP player skill level and heart rate. As pose success rate increases, heart rate increases as well, leading to better fitness outcomes.

\subsubsection{Game Design and Development with SNAP}

We designed and developed several games to demonstrate the capabilities of the SNAP system. Track Heroes relies on accelerometers placed on the hip and ankles to recognize steps and jumps. The game includes a $100 \mathrm{~m}$ dash, hurdles, and long jump mini-games. Figure 2.12 illustrates the two player hurdles game. 


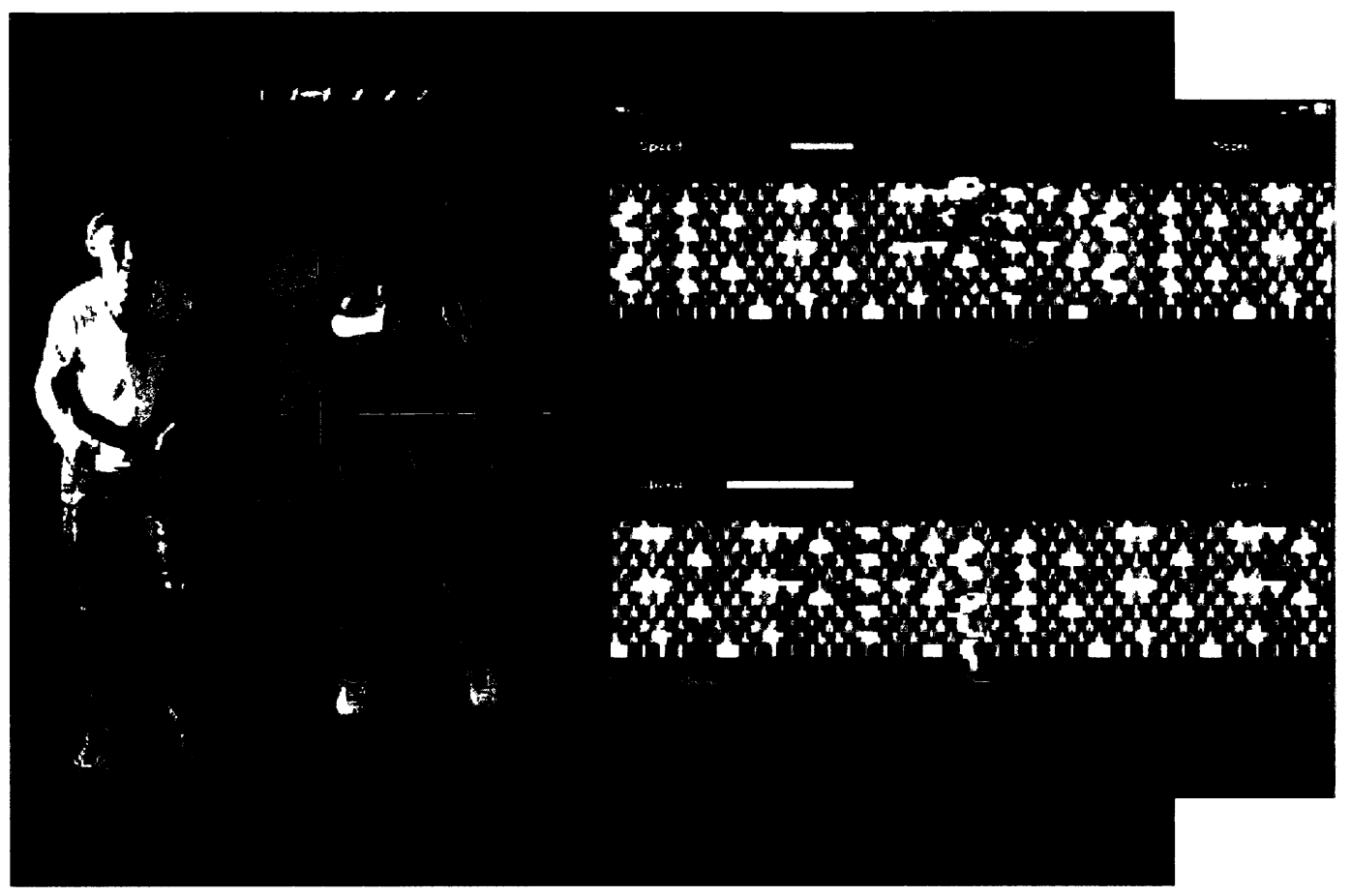

Figure 2.12 Users playing Track Heroes Hurdles (Left) and a game screenshot (Right)

The other games for the SNAP system involve replicating full-body dance poses. These pose-based games are the focus of this work and design features of these games are discussed in detail in the following subsections. 
Posemania requires players to perform dance poses to music at the right time. Poses scroll up the screen and players must replicate the pose as it reaches the top of the screen, in a similar fashion to Dance Dance Revolution (DDR).

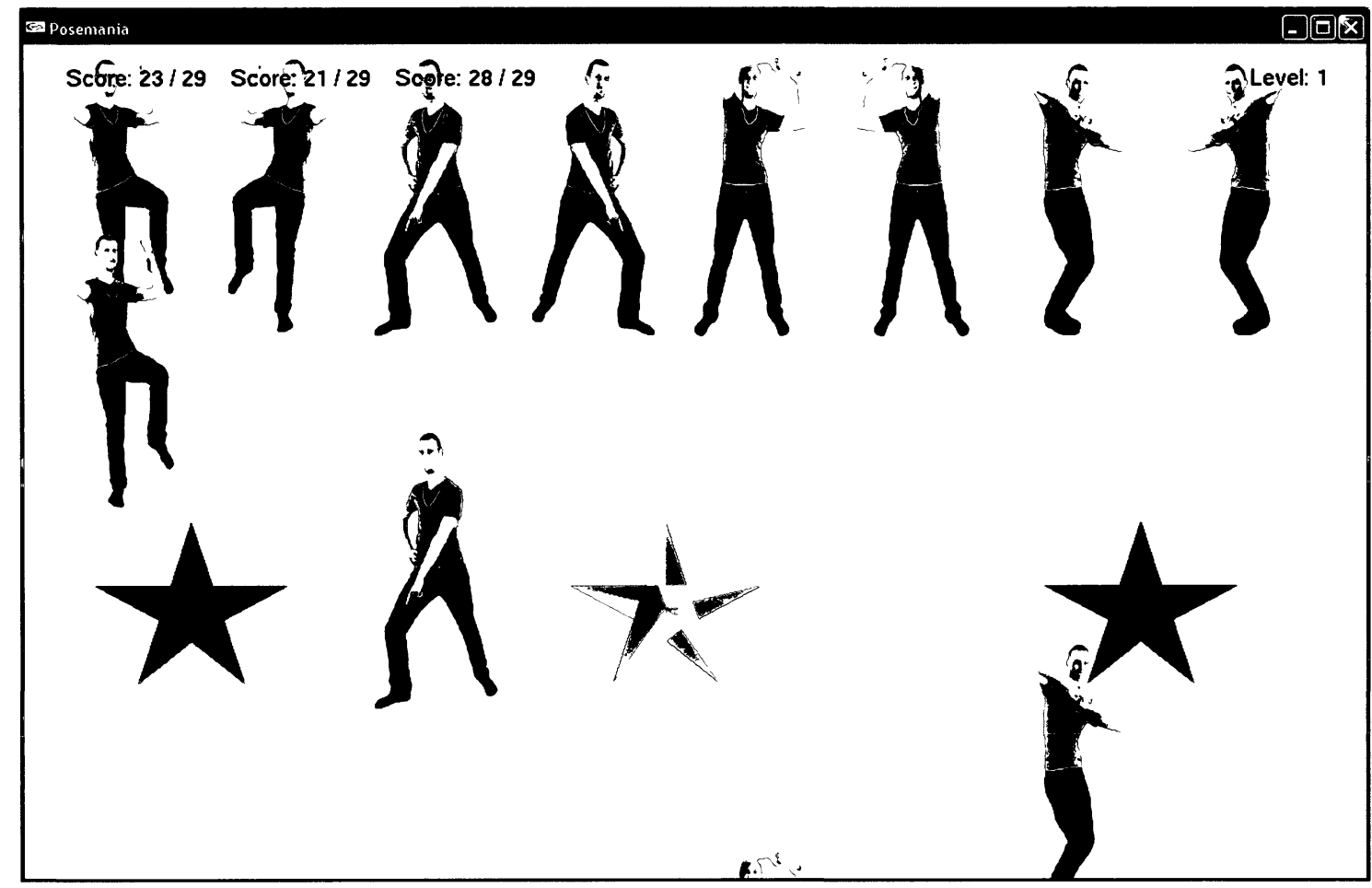

Figure 2.13 Screen shot of Posemania dancing game 
In RoboPaint, players must match the dance of an animated robot. As poses are correctly matched, the player's robot body gets painted. The player with the most successful poses, and thus painted parts, wins. Both Posemania and RoboPaint can be played with up to four players.

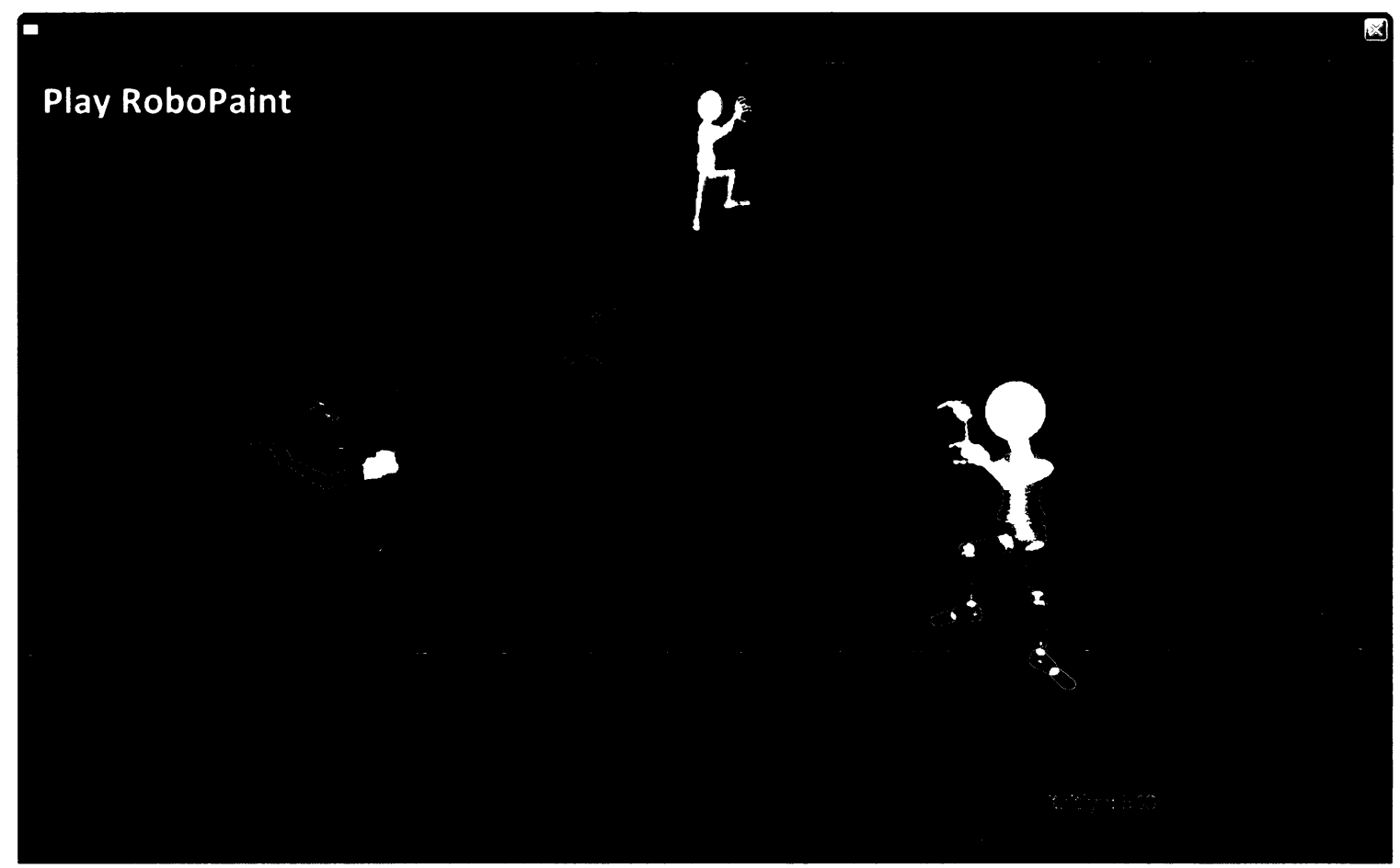

Figure 2.14 Screen shot of Posemania dancing game 
In Dance Fighter, players upload images of their own faces onto player bodies and danceoff against one another, using a series of predefined dance poses. For a duration they specify themselves, players must each perform the predefined dance moves in whichever, non-consecutive order they choose. Players earn more points for performing poses with higher difficulty ratings.

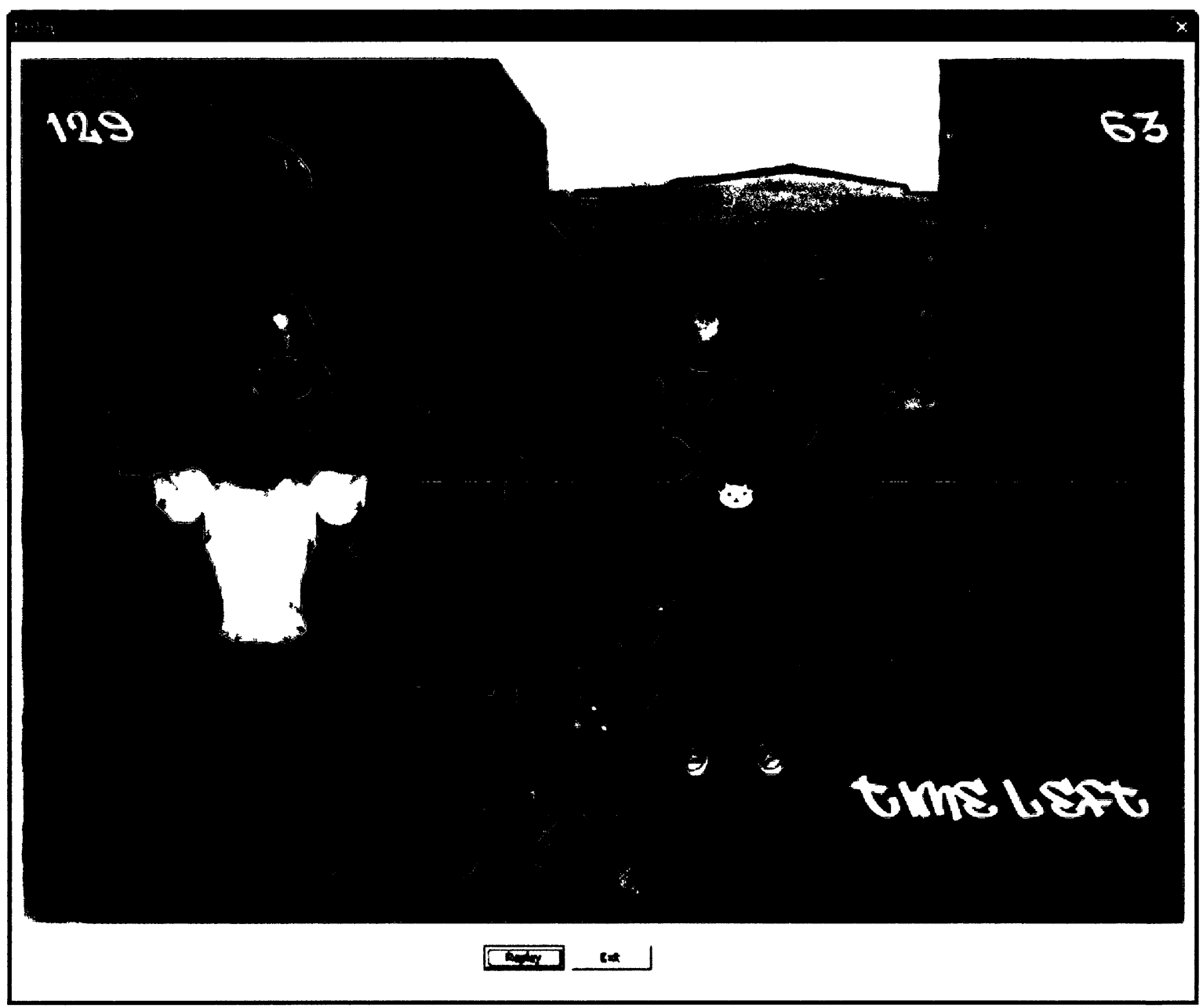

Figure 2.15 Dance Fighter game play 


\subsubsection{Persuasive Strategies}

In the current version of Posemania, players can monitor their success through a star graphic that becomes more colourful as the player get closer to perfecting the dance pose. A success score provides players with additional feedback and recognition. A threshold variable for pose accuracy can be adjusted to eliminate false negatives. Informal observation suggests that higher threshold values have the unfortunate consequence of making it significantly easier for players to perform all poses and can lead to "sloppy" dancing. The game also leverages social influence through multi-player competition.

\subsubsection{Player Enjoyment}

In a separate study, Posemania was tested with 62 young participants, aged 9-13 (29 male, 33 female). Participants played two at a time against each other at a speed of 40 PPM. In this version of Posemania, participants received feedback and additional score points for poses that more closely match the ideal pose. Participants were given group training on the system and the poses prior to playing the game once. They played for approximately one minute. The average response to "Did you enjoy the game?" (on a scale from 0-4) was 3.35, with no statistically significant difference between males (3.34) and females (3.37).

\subsubsection{Pose Selection}

To avoid confusion, it is generally desirable to select poses with obvious limb angles that can be seen with minimal image detail, for example, arms and legs forming 45, 90, or 180 degree angles. Complex limb positions are not only difficult to see on screen, but they tend to be more challenging to replicate. Each pose should be distinct from the others. It 
is important to recall that a change in yaw will not affect the acceleration values because gravity is still acting in the same direction. In games like Posemania, where the system checks the player's position against only one pose at a time, it is not critical for the poses to be distinct. In other games, similar poses may cause confusion or the occurrence of false positives.

\subsubsection{Pose Presentation}

It is important to illustrate the poses properly so players know what body position to replicate. A previous study suggests that most users find mirrored images easier to replicate [21]. Wii Fit makes use of both a view from behind and a rear-view style mirror image. In Posemania, 3-dimensional poses are represented by flat images in 2dimensional space, making it even more challenging to accurately convey body positions. For this reason, it is important to provide clear images of non-complex poses. In a modified version of Posemania, pose images were altered so that the left and right side of the body were each colour-coded. Through informal testing, we found that this change did not result in improved performance among experienced players; however, it is possible that the colour-coding might prove more useful for new users. Pose presentation is discussed further in section 4.4.

\subsubsection{Pose Speed and Precision}

We implemented two separate scoring systems in our prototype games. In the first system, players gain score points as they perform poses at or exceeding a given level of precision. Alternatively, players score an increasing number of points the closer they get to the ideal pose. Players receive visual feedback for their pose precision in the form of a 
change in colour brightness: the closer the participant is to perfectly replicating the pose, the brighter the hue of the star shape. Player observations suggest that the two separate scoring methods lead to different game play. With the first scoring system, players appear to do the minimum required to replicate the pose successfully. Players seem to be more concerned with speed to maximize the number of successful poses. With the additional precision requirement, players typically focus on carefully executing the pose perfectly and require more time per pose. The second method is generally preferred among new players as it provides more immediate feedback and satisfaction when they are still learning how to execute the poses. Both scoring methods are valid and each may be appropriate in different game contexts.

\subsubsection{Learning Multiple Poses}

Posemania requires players to learn and replicate eight poses, which can be overwhelming for some. The training process is more successful when players are able to see and try out the poses prior to game play. It may help users to start with a limited set of poses and gradually add new ones. Pose speed should also be increased gradually, initially allowing more time for players to recognize the pose and perform it correctly. When players must replicate poses at a speed beyond their capabilities, they often skip the most physically challenging poses. This reduces both enjoyment and health benefits from physical exertion. 


\section{Using SNAP in a Massively Multiplayer Online Exergame}

Informed by the exergame and persuasive technology literature, we have developed the concept of a massively multiplayer online exergame (MMOE) and identified critical design components for the proposed exergame. We present this work as a framework for an MMOE. The SNAP system is a viable input system for the game, meeting exertion, measurement accuracy, comfort, and accessibility requirements. We discuss ways that SNAP may be modified to better meet the needs of an MMOE, through feedback and credit mechanisms, appropriate levels of challenge, and customized and tailored elements. We also offer suggestions for how SNAP can be used to leverage social facilitation strategies, such as friendship and competition. We conclude with an overview of important privacy considerations for the system.

\subsection{Framework Summary}

Table 3.1 and Table 3.2 provide summaries of the framework for a massively multiplayer online exergame. Requirements are divided into physical input system and motivation and enjoyment aspects of the game. We propose combining exergames, persuasive technology, and massively multiplayer online games to deliver a more entertaining gaming experience that will consequently be a more successful long-term solution to physical inactivity. The tables below provide a summary of the framework for a massively multiplayer online exergame, divided into requirements for the physical input system and motivation and enjoyment aspects of the game. 


\begin{tabular}{|l|l|}
\hline Physical Exertion & $\begin{array}{l}\text { - Provide a flexible level of challenge } \\
\text { - Monitor and increase heart rate } \\
\text { - Incorporate leg or full-body movement }\end{array}$ \\
\hline $\begin{array}{l}\text { Measurement } \\
\text { Accuracy }\end{array}$ & $\begin{array}{l}\text { - Provide an appropriate level of accuracy for the application } \\
\text { - Supplement with additional sensors if additional accuracy is } \\
\text { necessary }\end{array}$ \\
\hline $\begin{array}{l}\text { Comfort of } \\
\text { Wearable Sensors } \\
\text { and Markers }\end{array}$ & $\begin{array}{l}\text { - Make on-the-body devices as small and light-weight as possible } \\
\text { - Make all devices wireless }\end{array}$ \\
& $\begin{array}{l}\text { Make input systems as inconspicuous as possible to limit } \\
\text { embarrassment }\end{array}$ \\
& - Plan for wear and durability through the use of replicable component \\
\hline
\end{tabular}

Table 3.1 MMOE Framework summary: physical input system

\begin{tabular}{|c|c|c|}
\hline \multirow[t]{5}{*}{$\begin{array}{l}\text { Individual } \\
\text { Factors }\end{array}$} & $\begin{array}{l}\text { Feedback, Credit } \\
\text { and Recognition }\end{array}$ & $\begin{array}{l}\text { - Provide on-going feedback } \\
\text { - Recognize positive behaviours and give appropriate credit } \\
\text { for accomplishments }\end{array}$ \\
\hline & $\begin{array}{l}\text { Challenge and } \\
\text { Flow }\end{array}$ & $\begin{array}{l}\text { Dynamically adjust level of difficulty to match player } \\
\text { skill level } \\
\text { - Leverage the flow state to maximize enjoyment and } \\
\text { satisfaction }\end{array}$ \\
\hline & $\begin{array}{l}\text { Tailoring and } \\
\text { Customization }\end{array}$ & $\begin{array}{l}\text { - Tailor the experience to individual needs and preferences } \\
\text { - Allow for creativity and customization of activity, avatars, } \\
\text { music, and other game content }\end{array}$ \\
\hline & Connectivity & $\begin{array}{l}\text { - Connect players to the Internet and other players to } \\
\text { provide targeted encouragement }\end{array}$ \\
\hline & Novelty & $\begin{array}{l}\text { - Leverage game novelty and ensure opportunities are } \\
\text { available to renew interest }\end{array}$ \\
\hline \multirow[t]{3}{*}{$\begin{array}{l}\text { Social } \\
\text { Factors }\end{array}$} & $\begin{array}{l}\text { Friendship and } \\
\text { Social } \\
\text { Facilitation }\end{array}$ & $\begin{array}{l}\text { Leverage the power of social facilitation to encourage } \\
\text { physical activity and positive behaviour change } \\
\text { - Foster positive social interaction and friendship building } \\
\text { in a safe online environment }\end{array}$ \\
\hline & Competition & $\begin{array}{l}\text { - Provide opportunities for competition among physically } \\
\text { close or distant friends and strangers } \\
\text { - Provide compensation mechanisms for fairness }\end{array}$ \\
\hline & Privacy & $\begin{array}{l}\text { - Use an online format to provide players with both } \\
\text { interactivity and anonymity } \\
\text { - Give users control over what they share online: } \\
\text { O Provide an option to hide their image } \\
\text { O Let users select who they interact with in the game }\end{array}$ \\
\hline
\end{tabular}

Table 3.2 MMOE Framework summary: motivation and enjoyment requirements 


\subsection{Physical Input System}

SNAP has been shown to provide players with the requisite physical exertion and measurement accuracy [13]. We discuss the adjustable, comfortable, and durable design of the sensor pods and straps and provide an overview of the conditions under which the SNAP system functions to support the greatest number of players and play environments.

\subsubsection{Physical Exertion}

The SNAP system is a physically engaging interface. In our previous work, Posemania was shown to encourage significantly higher intensity exercise than the Wii step and sedentary video games. Without specific attention to pose selection, Posemania provides enough intensity to elicit weight management benefits. Enforcing full-body activities is particularly effective for increasing physical exertion and energy expenditure [13]. The SNAP system is designed to support full-body poses, requiring players to move all of their limbs. The degree of difficulty, and consequently physical exertion, can be adjusted based on the needs or goals of the player. This can be achieved by enforcing a greater degree of pose accuracy or increasing the speed. Competition among players can also contribute to player exertion. Players can determine the appropriate challenge themselves, or level of difficulty can be dynamically adjusted based on additional information from the user, such as heart rate or pose success rate.

\subsubsection{Measurement Accuracy}

Activity-sensing input systems are becoming increasingly common and sophisticated. It is likely that in the near future, systems will become more accurate and less expensive. 
The SNAP system is a viable response to the needs outlined in the previous chapter. While not an ideal solution for all types of activity measurement, the accelerometer sensor network provides accurate, full-body pose recognition. With proper training, and a reasonable training set size, recognition rates can approach $100 \%$. While self-training is helpful in achieving extremely high levels of accuracy, it is certainly not necessary. As the number of online players grows, the pose bank becomes sufficiently generalized and eventually new players no longer have to retrain poses. Cheaters and outliers can easily be removed programmatically.

Additional accelerometers or supplementary sensors, such as gyroscopes, dramatically reduce or even eliminate yaw-axis limitations of the system. One important limitation of the accelerometer sensor network is that it is most effective for static pose recognition, requiring players to momentarily freeze in place. A more complicated system for movement and gesture recognition is possible using the SNAP system, but results suggest that it is less accurate than static pose recognition [96]. Thus, depending on the requirements of the game interaction, a homogeneous accelerometer network may or may not be appropriate, but it has shown to be extremely well suited to dance-based games.

\subsubsection{Comfort of Wearable Sensors and Markers}

The SNAP system involves small, lightweight sensors, which are contained in robust sensor pods (detailed in section 2.4.2.1) that help protect the accelerometers against damage from repeated movement, accidental dropping, and other wear and tear. The pods attach to the body using flexible, adjustable hook and loop straps. The straps slip through the sensor pods, so players can detach the sensors and wash the straps separately, while 
the plastic pods can be easily wiped clean. The size of the sensor straps is of particular importance for accurate pose training and recognition. One early (and flawed) solution involved placing straps that were too large higher up on the forearm. At first glance, it would seem as though acceleration measurements would be the same with the pod placed anywhere along the forearm; however, as the wrist and arm rotate, they do so at varying amounts. The challenges are illustrated in Figure 3.1. Fortunately, a simple adjustment to strap length, or providing players with straps of different sizes, allows players to position the sensor correctly, thus resolving the issue completely.
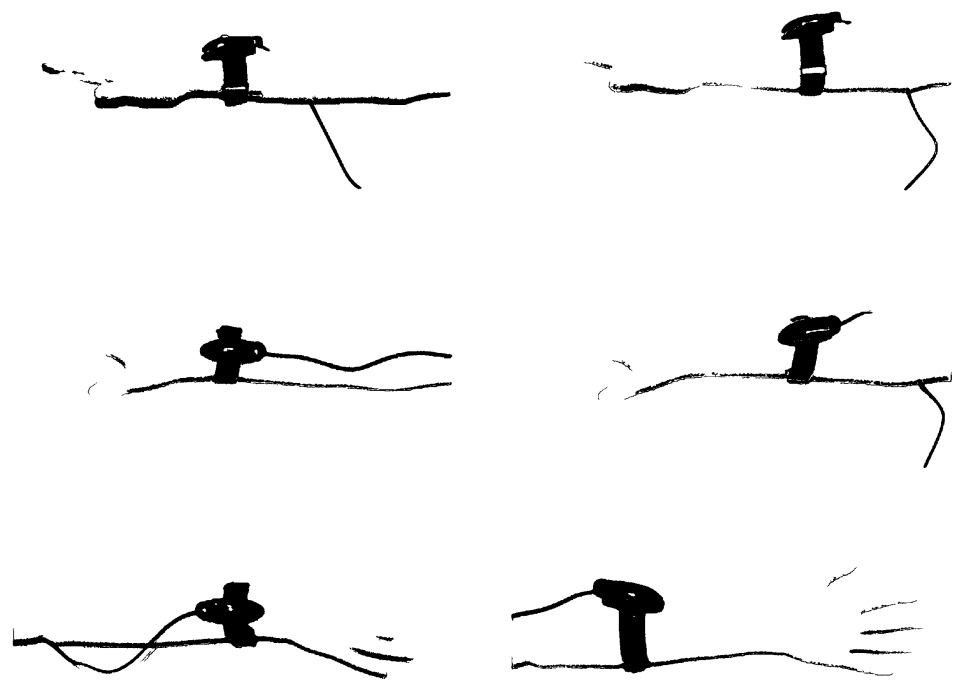

Figure 3.1 Sensor rotation angle as the pod moves from wrist to forearm

While the current version of the system uses accelerometers, attached with USB cords, wireless devices are possible. The sensors are quite visible, but it is reasonable to assume that as sensors decrease in size, players could wear the SNAP system unnoticed, or even embedded into regular clothing [29]. In the present condition, participants may wear whatever clothing they choose while using the SNAP system. 


\subsubsection{Accessibility and Convenience}

The SNAP system is ideally suited to home use. The sensors can be worn with any clothing, be it for a serious workout, or casual play. No special space arrangements are necessary, provided players have enough room to move their arms and legs into poses. The SNAP system can be used in any lighting conditions, even complete darkness. The sensors are inexpensive enough for home use, allowing players to participate in the exergame at any time of day or night. The simplicity of the SNAP system requires limited setup and configuration.

\subsection{Motivation and Enjoyment}

In this section, we discuss how the SNAP system leverages the two main branches of motivation and enjoyment: individual and social factors.

\subsubsection{Individual Factors}

The SNAP system supports individual motivation elements including feedback and recognition, challenge and flow, tailoring and customization, and novelty. These critical exergame features are described in detail in the following subsections.

\subsubsection{Feedback and Recognition}

The detailed acceleration information collected from the SNAP system makes it possible to provide players with targeted feedback. In the current version of Posemania, players only receive feedback about the overall accuracy of the pose. It would be possible, and likely more valuable, to give users information as to which limb, or limbs, contribute most to the pose error. Feedback could be presented via text, speech, or visualized by 
highlighting the part of body in the on-screen image. With consideration of appropriate language, additional text could also provide players with useful and motivating information about his or her progress. Players should be provided with recognition for their success, both at the pose and dance level. Using the SNAP system, success information could be provided both at the dance (or game) level, as well as the pose, and even individual limb level, providing players with the tools necessary for improvement.

\subsubsection{Challenge and Flow}

The SNAP system encourages flow in several ways, including dynamic speed, pose classification threshold, and pose selection. All of these elements are easily modifiable and contribute to the level of difficulty of the games. In Dance Dance Revolution, speed is the main challenge players face as they progress through game levels and it is an especially easy modification to make. Setting the appropriate speed can also have a great impact on the players' level of exertion. If the game requires players to move faster than they are able, they may give up. However, in a flow state, players will exert themselves more, without necessarily feeling as though they are exerting themselves. The SNAP system permits variability of both speed and strictness of pose enforcement to provide players with an appropriately challenging experience. It may be more enjoyable for inexperienced players to play with a loose threshold, permitting some degree of sloppy posing. As players become familiar with the game requirements and poses, strictness can be increased.

With the SNAP system, challenge may be introduced through deliberate pose selection. Game designers can select poses that require varying amounts of body movement and 
muscle use. For example, squats, torso shifts, and leg lifts are significantly more strenuous than arm movements alone. In future versions of the game, when players can select and train their own poses, players could also assign a level of difficulty to the moves. Alternatively, it may be possible to devise a metric to determine this automatically. By knowing the relative difficulty of each pose, and possibly each pose combination, the game can dynamically select the frequency of these poses and combinations that maximizes player flow.

\subsubsection{Tailoring and Customization}

By collecting some additional user information, such as age, weight, and heart rate, the SNAP system could better tailor the level of difficulty to individual users in order to optimize player flow and enjoyment. The system could collect information about the player's success to dynamically adjust the level of difficulty and maximize his or her enjoyment and exertion. Similarly, the system might alter game play to take into consideration the player's frequency or duration of play. More sophisticated tailoring strategies draw on affective computing research, taking into consideration factors such as the player mood, environment, or aesthetic taste.

SNAP provides many opportunities for player creativity and customization. The most obvious is through the creation of dances and individual poses. Players can also add music of their choosing. Players can choose to create dances that are physically challenging, artistic, or simply fun. The possibilities are endless and the simplicity of the sensor network does not constrain users. Furthermore, the SNAP system does not in any 
way interfere with other avenues for in-game creativity, such as avatar personalization or modification and creation of other interactive or environmental components.

\subsection{Activity}

We have previously shown that the SNAP system lends itself to a variety of activity types [11]. In the context of a dance-based MMOE, the accelerometer sensor network is capable of training and recognizing a wide variety of pose types. Player creativity is not restricted by the capabilities of the hardware.

\subsection{Avatars}

The SNAP system does not have a significant impact on the selection and modification of game avatars, however, consideration should be given to the game interface in order to facilitate the process. The current version of the SNAP sensor pods do not come equipped with any controller-like buttons, and as such, more complex game interactions (such as avatar design) may require a different input mechanism.

\subsection{Music}

The current version of Posemania for the SNAP system allows players to select music of their choosing. While song selection may impact individual player motivation, the SNAP system does not currently alter game play based on musical qualities.

\subsection{Connection to a Network}

The current version of the SNAP system does not leverage a network, however, as the system relies on relatively limited data transfer, SNAP may be integrated into an online game in the foreseeable future. 


\subsection{Novelty}

The methods of customization previously described suggest a number of ways that the SNAP system may be used to provide players with a constantly evolving and novel gaming experience. The most significant source of novelty using the SNAP system is the possibility for players to generate a near infinite number of unique dance poses and dances. Players can create dances for their favourite new music or modify dances based on what they have seen elsewhere, in a sense perpetually 'remixing' the exergame.

\subsubsection{Social Factors}

Social factors are an extremely important part of the MMOE. In this section, we describe the ways in which the SNAP system can be used to facilitate friendship, social facilitation, competition, and privacy protection.

\subsubsection{Friendship and Social Facilitation}

Friendship and social facilitation are key motivations behind the concept of the MMOE. SNAP has very little impact on the social experience for players online beyond providing the technology necessary for players to create and share their own dance poses. The design of the SNAP sensors also allows players to share the equipment with friends or family members, expanding the pool of players and improving the overall gaming experience. Co-located players can help and encourage one another to perform more challenging poses or increase their pace. 


\subsubsection{Competition}

Players already compete against each other in all of the games created for the SNAP system, but social influence could play a huge role in motivating players to continue playing once the initial novelty of the system wears off. A networked version using SNAP as input would allow friends, or even distant strangers, to compete against one another. These virtual dance-offs could occur across the globe, from the convenience of players' own homes. In an MMOE, players could send each other competition invitations, suggesting and encouraging more frequent play.

Though not implemented, we foresee a variety of compensation mechanisms to increase perceived fairness in competition among players of widely varying skill levels. The system might demand a higher degree of precision or a faster response rate from a more experienced player. Another solution would be to provide players with suggestions of opponents with comparable skill levels.

\subsubsection{Privacy}

One of the most promising features of the MMOE is that it can provide both interactivity and anonymity. The physical appearance of the player using the SNAP system remains unimportant, giving some users, who might otherwise be shy or embarrassed, the opportunity to participate actively. Using the SNAP system, players can choose to play with real world friends or complete strangers online. Players could select or design their avatar to resemble themselves as closely as they are comfortable. No cameras are required for actual game play with the SNAP system and the accelerometer network- 
based pose recognition system relies only on acceleration data, giving players the option for full anonymity. However, the use of cameras may be desirable for sharing and communicating poses to other players. Alternate methods of pose capture for display are discussed in the following chapter. 


\section{Development of a Customizable Dance Pose Creation System}

There are many possible approaches to pose selection, capture, and training. We evaluate two strategies for pose selection within the proposed dance-based exergame system in this section. Next, we discuss the challenges involved in pose capture and propose two alternate solutions. We then provide an overview of issues related to pose training and how the process may be tackled, specifically in the context of a massively multiplayer online exergame. The chapter concludes with an investigation into pose presentation methods.

\subsection{Pose Selection}

The first issue in developing a dance-based exergame system is pose selection. Two broad categories of pose selection exist: automated pose selection and user-controlled selection. The selection methods are compared in Table 4.1 below. 


\begin{tabular}{|c|c|c|}
\hline Selection Method & Advantages & Disadvantages \\
\hline $\begin{array}{l}\text { Automated Pose } \\
\text { Selection }\end{array}$ & $\begin{array}{l}\text { Captures representative poses } \\
\text { - Captures spontaneity, surprise, } \\
\text { serendipity } \\
\text { - Users could select from many } \\
\text { possible poses } \\
\text { Many selection options are also } \\
\text { possible (extracting poses at } \\
\text { timed intervals, or based on } \\
\text { tempo, expression, position) } \\
\text { - Limited effort from users }\end{array}$ & $\begin{array}{l}\text { Continuous motion makes the } \\
\text { system more vulnerable to motion } \\
\text { blur, sensor disruption, and } \\
\text { disorganized or messy poses in } \\
\text { capture } \\
\text { - Freestyle dancing may be } \\
\text { awkward or embarrassing for } \\
\text { some users } \\
\text { - Some amount of user intervention } \\
\text { for filtering is likely necessary }\end{array}$ \\
\hline $\begin{array}{l}\text { User-Controlled } \\
\text { Pose Selection }\end{array}$ & $\begin{array}{l}\text { - Controlled environment may be } \\
\text { less embarrassing } \\
\text { - Limited motion interference } \\
\text { with camera images or sensors } \\
\text { - May be possible to combine } \\
\text { pose selection with pose capture } \\
\text { and training }\end{array}$ & $\begin{array}{l}\text { - Requires more user planning } \\
\text { - Requires more system interaction, } \\
\text { users can't "set-it-and-forget-it" }\end{array}$ \\
\hline
\end{tabular}

Table 4.1 Comparison of automated and user-controlled pose selection methods

\subsubsection{Proposed Solution}

Given the importance of customization to the overall success of the MMOE, a usercontrolled approach to pose selection would likely be more successful in the majority of circumstances. However, in situations involving less computer-savvy users, or dancers with a more improvised style, an automated system could be beneficial. It should also be noted that both methods could be offered to users, giving players even more control over pose selection.

\subsection{Pose Capture for Display}

Once poses have been selected, players must share them with other players. In the context of an MMOE, players do not have the luxury of in-person explanations and demonstrations. To capture the poses, one approach is to use a 2-dimensional or 3dimensional model avatar to represent the full-body dance poses. A simple version could provide full joint rotations and limb movements, while a more sophisticated model might 
constrain the joints and limbs based on physiological limitations of the human body.

Another solution uses a network of web cameras to capture real images of the poses.

Multiple cameras may be used together to capture the full body pose and depth information. A comparison of the two capture methods is included in Table 4.2.

\begin{tabular}{|c|c|c|}
\hline Capture Method & Advantages & Disadvantages \\
\hline Poseable Avatar & $\begin{array}{l}\text { - Provides full anonymity } \\
\text { and privacy } \\
\text { - Players can express } \\
\text { themselves without } \\
\text { providing any personal } \\
\text { information } \\
\text { - Could offer a range of } \\
\text { customizable features } \\
\text { - The avatar system could be } \\
\text { supplemented with sensor } \\
\text { data, or use a one-to-one } \\
\text { mapping combining of } \\
\text { accelerometers and } \\
\text { gyroscopes, requiring } \\
\text { players to simply verify and } \\
\text { correct a pose, alleviating } \\
\text { much of the work for users }\end{array}$ & $\begin{array}{l}\text { - Model must be reasonably accurate } \\
\text { and detailed in order to move } \\
\text { realistically } \\
\text { - An intelligent system requires more } \\
\text { complex development } \\
\text { - Different models are necessary to } \\
\text { accommodate different genders and } \\
\text { body types } \\
\text { - Players may be more likely to } \\
\text { generate impossible poses } \\
\text { - Subtleties of pose and expression } \\
\text { may be lost } \\
\text { - Requires some amount of manual } \\
\text { input, for which a more complex } \\
\text { interface is necessary to allow users } \\
\text { to manipulate the avatar }\end{array}$ \\
\hline $\begin{array}{l}\text { Image-Based } \\
\text { Recording System }\end{array}$ & $\begin{array}{l}\text { Provides an accurate } \\
\text { representation of full body } \\
\text { poses, with limited effort on } \\
\text { the part of the user } \\
\text { - Once the cameras are } \\
\text { registered, recording } \\
\text { multiple poses is quick and } \\
\text { easy } \\
\text { The system constrains } \\
\text { players to feasible body } \\
\text { positions }\end{array}$ & $\begin{array}{l}\text { A large, bright, cleared space is } \\
\text { required } \\
\text { - Players are confined to a specific } \\
\text { distance from the cameras } \\
\text { - The addition of cameras to the } \\
\text { gaming system increases the cost, } \\
\text { setup, and calibration for players } \\
\text { - Using real player images introduces } \\
\text { privacy concerns, particularly when } \\
\text { intended for online use }\end{array}$ \\
\hline
\end{tabular}

Table 4.2 Comparison of poseable avatar and image-based pose selection methods

\subsection{Pose Training}

The MMOE could rely entirely on community members to train the poses, intervening programmatically only to remove outliers, cheaters, or other instances of problematic data. Over time, the data becomes an increasingly accurate representation of the dance 
poses provided. In the current versions of the dance-based SNAP games, a training bank of approximately 5-8 sets of data is used. We have previously shown that this number of unique participants contributing data to the pose bank provides a sufficiently generalized set of pose data [10]. However, to accommodate the widest range of player shapes and sizes, a greater amount of pose data could be collected by requiring members of the online community to train poses. This could go so far as to require every player to train the poses for every dance they play, however, this strategy would result in diminishing returns fairly quickly. It may be desirable to provide players with unique body sizes or shapes the option of re-training the poses.

\subsection{Pose Presentation}

In an MMOE, players would design their own poses and share them with others. Players require some graphical representation of the full body poses, in order to know precisely what to replicate in the game. Ideally, players should be able to mimic the pose quickly and accurately from whatever presentation method is chosen. Several different presentation strategies were developed and tested in an attempt to determine the qualities that provide players with the most useful visual description of the poses. In the game, pose presentation serves more as a reminder or visual stimulus to action, and with practice, it is probable that players could adapt to any presentation method. However, by providing players with the most intuitive presentation possible, we allow players to focus on more entertaining and enjoyable aspects of the game early on. We describe the presentation testing methods next. 


\subsubsection{Pose Presentation Testing}

The purpose of pose presentation testing is to determine the most effective method of visually describing poses from afar, so that participants can replicate poses they encounter online, without direct human intervention or coaching on proper limb positioning.

\subsubsection{Test Procedure}

The test procedure was as follows:

1. Welcome participant

2. Provide a brief overview:

a. Participant will be shown images of full-body poses, one at a time on screen.

b. As soon as participant sees the pose, participant attempts to replicate it as quickly and accurately as possible

c. When participant thinks he/she is in the right position, participant notifies the experimenter

d. The mouse is clicked and the pose image disappears

e. Participant should break out of the pose and relax

f. About five seconds later, the next pose appears

g. The process repeats five times (for a total of six different poses)

h. Participant is asked if he/she has any questions, and any necessary clarification is provided

3. Help participant attach sensors 
4. Double check that sensors are positioned and connected properly

5. Start program

6. Assign sensors

7. Fill in filename and participant name

8. Inform the participant that the first pose will be used to explain and demonstrate the representation method (mirrored, non-mirrored, front + back view, etc).

9. Tell the participant which method they will use and start by showing them Pose 0 (wait to click until they are ready to begin)

10. Go through the five other poses

11. Ask participant to fill out the survey

12. Thank participant

\subsubsection{Test Conditions}

The five presentation methods (illustrated in Figure 4.1) include direct (D), mirrored (M), mirror and back (MB), mirrored and side (MS), and mirrored with feedback (MF) views. The direct (D) view requires participants to put themselves in the place of the person in the image. The photo is of a person raising her right arm, so the participant also raises his or her right arm. In the mirrored (M) view, participants act as though they are looking at themselves in a mirror. The left arm of the person in the photo is raised, so the participant raises his or her right arm. The mirrored and back (MB) presentation gives players an additional view from behind. The back view remains non-mirrored, as though the player is watching him or herself. The back view is comparable to many third-person views in games. In the mirrored and side (MS) view, players receive an additional view from the side. The side view remains non-mirrored, as though the player is watching him or herself 
from the side. In the mirrored with feedback (MF) view, the mirrored image is overlaid with four coloured quadrants. The farther the player is from the ideal pose, the more the image is overlaid with the colour red. This distance or error is presented separately for each limb sensor. As the player approaches the ideal pose, the picture gets closer to greyscale.

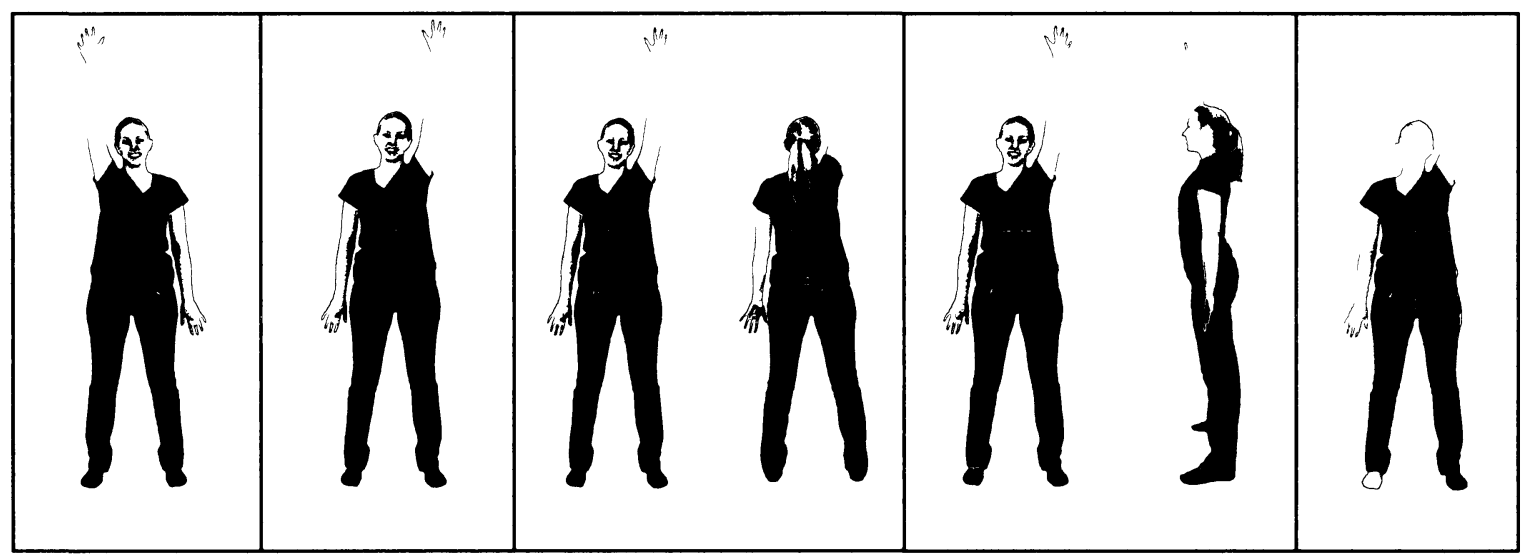

Figure 4.1 Presentation conditions (left to right) direct, mirrored, mirrored and back, mirrored and side, and mirrored with feedback

\subsubsection{Test Poses}

Five poses and one practice pose were selected for testing purposes. The poses vary in symmetry, complexity, and difficulty. Consideration was also given to side-dominance, with some of the poses favouring the left side, and others the right. Figure 4.2 shows all six poses, from the direct view perspective. 


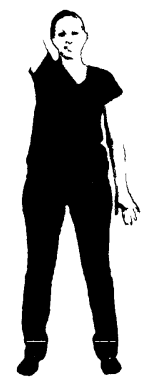

(0)

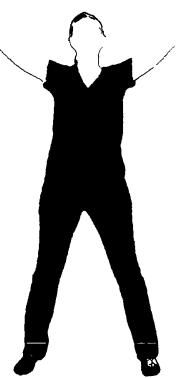

(1)

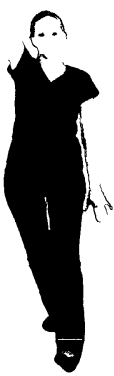

(2)

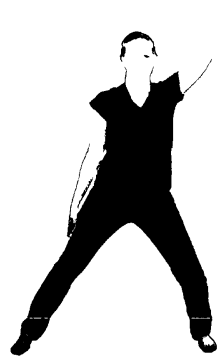

(3)

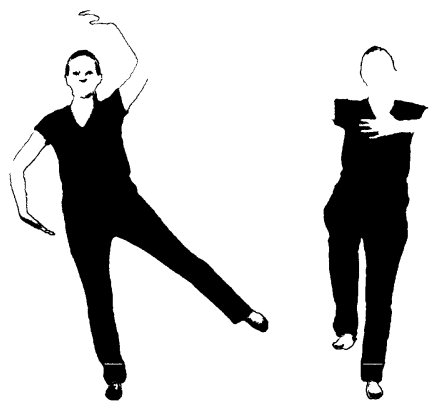

(4)

(5)

Figure 4.2 Practice pose (pose 0 ) and five poses for presentation method testing

\subsubsection{Test Pose Training Bank}

For the purposes of testing, a pose training bank was created with data from three females and two males. They range in age from 23 to 37, with an average age of 26.8. Participants in the training set have an average height of $169.8 \mathrm{~cm}$ with a range of $162 \mathrm{~cm}$ to $175 \mathrm{~cm}$. Their average weight is $75.7 \mathrm{~kg}$, ranging from $62 \mathrm{~kg}$ to $118 \mathrm{~kg}$.

\subsubsection{Test Participants}

We conducted two separate tests, one of young adults and the other of older adult women. Detailed information on the two separate populations is provided in the following sub-sections.

\subsection{Young Adult Participants}

The young adult test population consisted of 30 female and 30 male participants. Participants were distributed evenly between the five presentation conditions (direct, mirrored, mirrored and back, mirrored and side, and mirrored with feedback), with 12 participants in each. The male to female ratio of each condition was approximately equal, 
ranging from five to seven males and five to seven females per presentation method. Table 4.3 provides an overview of participant age, height, and weight information, as reported by the participants.

\begin{tabular}{|c|c|c|c|}
\hline & Average & Low & High \\
\hline Age & 20.5 & 18 & 27 \\
\hline Height & $170.1 \mathrm{~cm}$ & $152 \mathrm{~cm}$ & $193 \mathrm{~cm}$ \\
\hline Weight $^{*}$ & $65.4 \mathrm{~kg}$ & $43 \mathrm{~kg}$ & $100 \mathrm{~kg}$ \\
\hline Fitness Level (1-5) & 3.2 & 1 & 5 \\
\hline Video Game Experience (1-5) $^{-1}$ & 3.7 & 1 & 5 \\
\hline
\end{tabular}

Table 4.3 Young adult participant characteristics

* Three participants declined to report their weight and were consequently excluded from that measure. The remaining characteristics include data from all 60 participants.

\subsection{Older Female Participants}

A separate test was conducted with eight female participants. Due to the limited sample size, only the mirrored and mirrored with feedback presentation methods were compared. Table 4.4 provides an overview of participant age, height, and weight information, as reported by the participants.

\begin{tabular}{|c|c|c|c|}
\hline & Average & Low & High \\
\hline Age & 62.8 & 49 & 73 \\
\hline Height & $162.9 \mathrm{~cm}$ & $155 \mathrm{~cm}$ & $175 \mathrm{~cm}$ \\
\hline Weight* $^{*}$ & $68.7 \mathrm{~kg}$ & $59 \mathrm{~kg}$ & $85 \mathrm{~kg}$ \\
\hline Fitness Level (1-5) & 2.8 & 2 & 4 \\
\hline Video Game Experience (1-5) $^{-1.5}$ & 1 & 2 \\
\hline
\end{tabular}

Table 4.4 Older adult female participant characteristics

* One participant declined to report her weight and was consequently excluded from that measure. The remaining characteristics include data from all 8 participants. 


\subsubsection{Pose Presentation Results}

In this section, we provide a summary comparison of the five presentation conditions, including differences in speed required to complete the pose, accuracy of the pose completed, and perceived difficulty of pose replication. Pose 0 was omitted from all results as it was used strictly for practice and clarification.

\subsection{Effect of Presentation Method on Speed}

Speed varied significantly between several presentation methods. In this experiment, we define speed as the time taken by participants to replicate a pose, indicate to the experimenter, and for the experimenter to register the completion with a mouse click. Speed consequently includes reaction time of the experimenter, which is assumed to be constant across all participants. The fastest average time was the mirrored and back view, but it was not significantly faster than mirrored or mirrored and side views. The mirrored with feedback method was statistically slower than all other conditions ( $\mathrm{P}$ values of 0.008, 0.001, $0.001,0.004$ for direct, mirrored, mirrored and back, and mirrored and side respectively). Mirrored, and mirrored and back views had faster pose times than the direct method, with statistical significance ( $\mathrm{P}$ values 0.005 and 0.001 respectively). The direct presentation method seems to require more mental processing time. These speed differences and their implications are explained in more detail in the section Effect of Presentation Method on Perceived Difficulty. A summary of the speed statistics, broken down by presentation method, is included in Table 4.5. Q1 is the median of the lower half of values, representing the bottom $25 \%$ of the data, while Q3 is the median of the upper half, representing the top $25 \%$. 


\begin{tabular}{|c|c|c|c|c|c|}
\hline Speed Statistic (s) & D & M & MB & MS & MF \\
\hline Sample Mean & 5.7 & 4.2 & 4.0 & 4.8 & 10.7 \\
\hline Median & 6.0 & 4.1 & 3.7 & 4.6 & 9.2 \\
\hline Sample Standard Deviation & 1.2 & 1.1 & 0.8 & 1.7 & 5.8 \\
\hline Maximum & 7.2 & 6.6 & 6.0 & 7.7 & 20.0 \\
\hline Minimum & 3.5 & 2.9 & 3.2 & 2.5 & 3.1 \\
\hline Q3 (0.75) & 6.5 & 4.8 & 4.4 & 5.6 & 14.5 \\
\hline Q1 (0.25) & 5.1 & 3.5 & 3.4 & 3.5 & 6.9 \\
\hline
\end{tabular}

Table 4.5 Statistics describing speed by presentation method

Supplementing the straight-on mirrored image with additional viewpoints, such as the back and side views, does not appear to significantly improve the response times of the participants. Confusion may arise from the juxtaposition of a mirrored and a nonmirrored view. This may be better addressed by dynamic views, as discussed later. Figure 4.3 illustrates the differences in speed of the presentation methods.

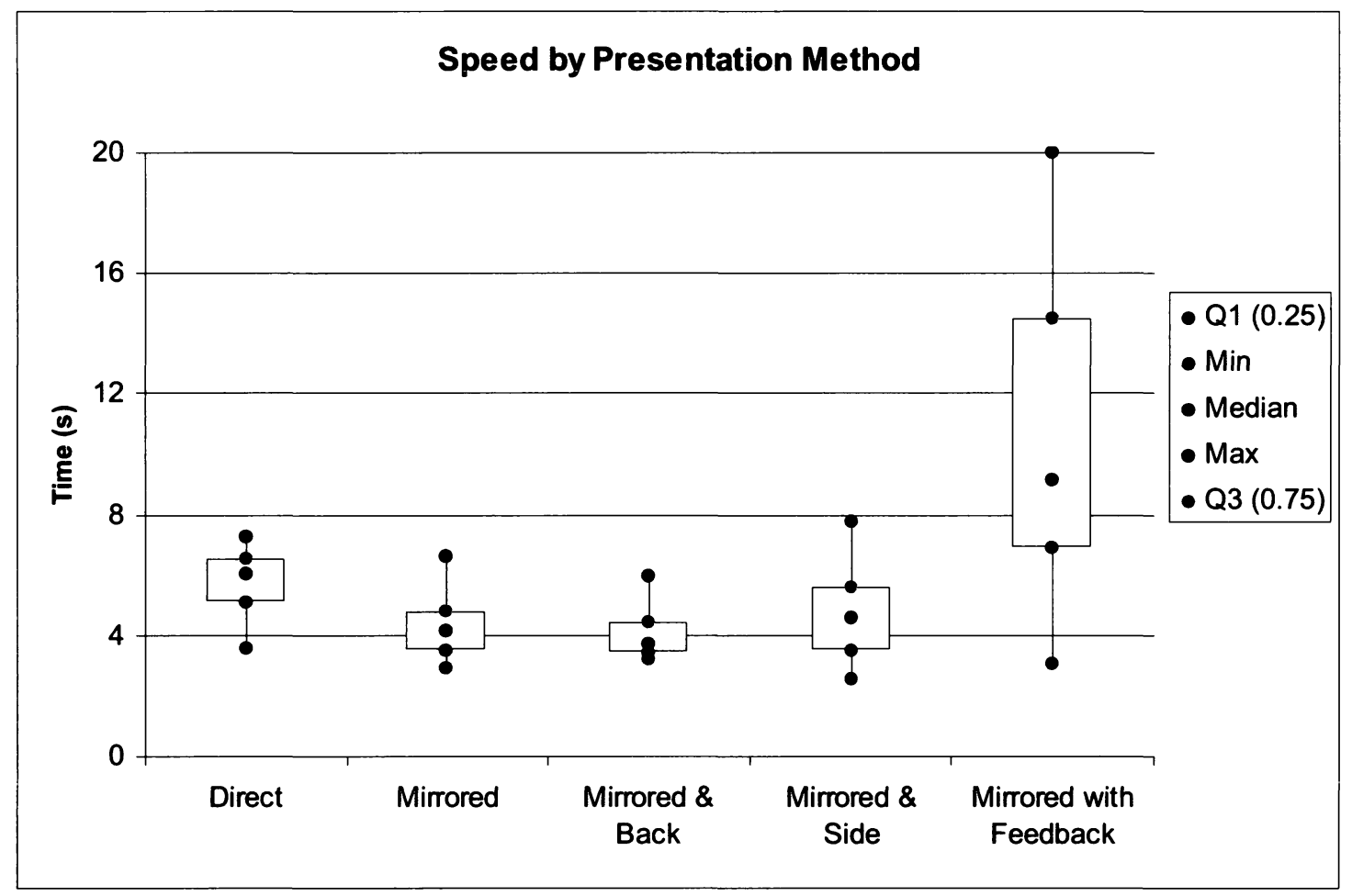

Figure 4.3 Speed by presentation method 
Additional information can be gleaned by breaking down speed information by individual pose, as shown in Table 4.6 and Figure 4.4. The shortest times per pose are highlighted.

\begin{tabular}{|c|c|c|c|c|c|}
\hline \multirow{2}{*}{ Pose } & \multicolumn{5}{|c|}{ Time (s) } \\
\cline { 2 - 6 } & D & M & MB & MS & MF \\
\hline $\mathbf{1}$ & 3.0 & 3.1 & $\mathbf{2 . 9}$ & 3.1 & 13.1 \\
\hline $\mathbf{2}$ & 5.8 & 5.0 & $\mathbf{4 . 7}$ & 5.9 & 9.3 \\
\hline $\mathbf{3}$ & 6.1 & 4.0 & $\mathbf{3 . 8}$ & 5.1 & 12.0 \\
\hline $\mathbf{4}$ & 7.5 & 4.9 & $\mathbf{4 . 3}$ & 5.2 & 10.0 \\
\hline 5 & 5.8 & 3.8 & 3.4 & 4.2 & 9.4 \\
\hline
\end{tabular}

Table 4.6 Average time to pose, broken down by individual pose

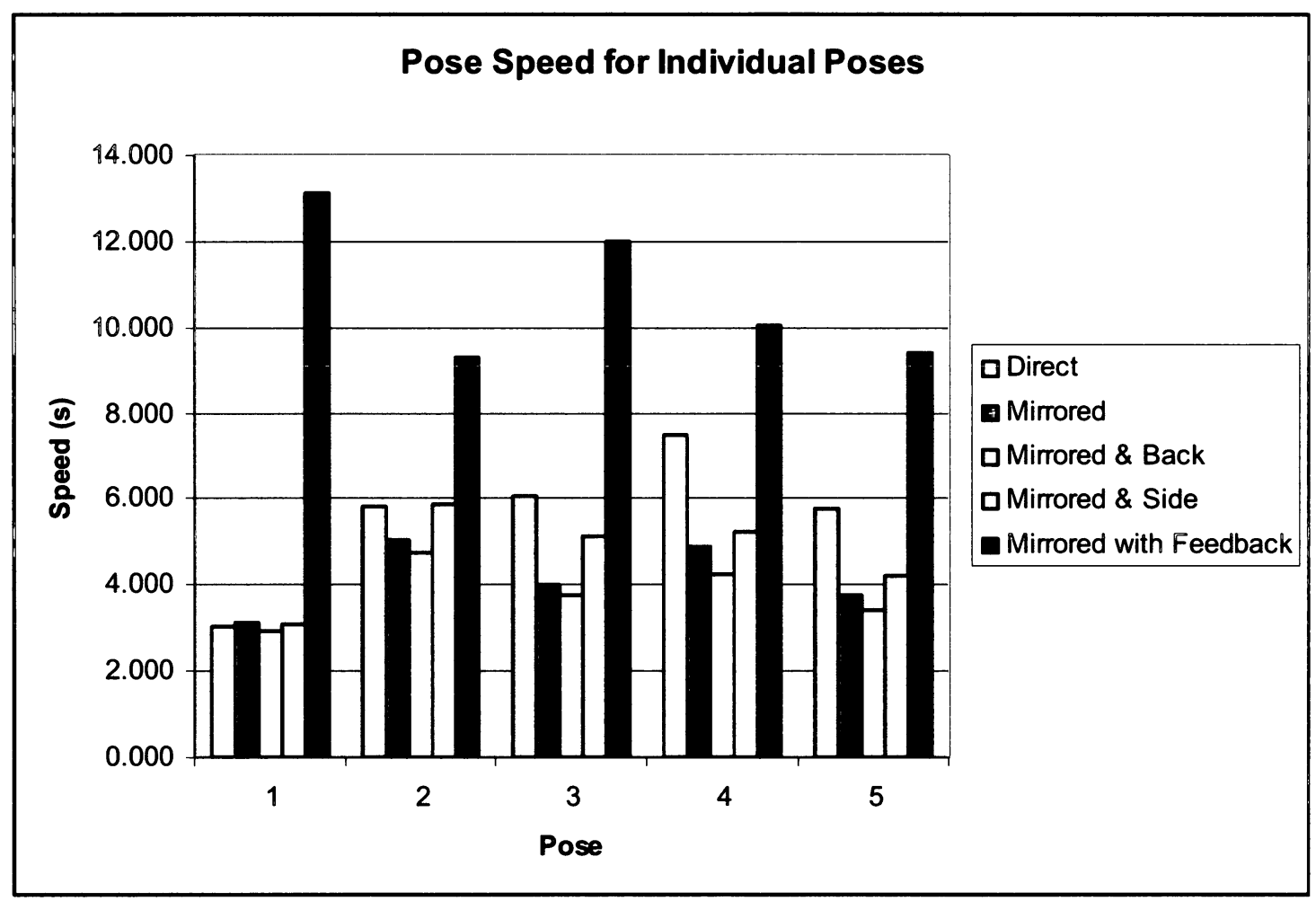

Figure 4.4 Breakdown of the speed required for each pose

With the exception of the mirrored with feedback method, the time values for pose 1 are remarkably close. Pose 1 is a symmetrical pose, which further supports the theory that differences in time are the result of processing time required to mentally flip the images. 
The other four poses follow a fairly consistent pattern for each of the presentation methods.

\subsection{Effect of Presentation Method on Accuracy}

Table 4.7 includes a summary of the statistics describing accuracy for each of the five conditions. The lower the Mahalanobis distance, the more accurately participants were able to replicate the poses.

\begin{tabular}{|c|c|c|c|c|c|}
\hline Distance Statistic (Mahalanobis) & D & M & MB & MS & MF \\
\hline Sample Mean & 6.7 & 5.7 & 5.7 & 6.3 & 6.1 \\
\hline Median & 5.8 & 5.8 & 5.8 & 5.5 & 5.8 \\
\hline Sample Standard Deviation & 2.0 & 1.9 & 1.1 & 2.9 & 0.9 \\
\hline Maximum & 10.3 & 10.0 & 7.1 & 15.0 & 8.3 \\
\hline Minimum & 4.4 & 3.3 & 4.0 & 4.4 & 5.0 \\
\hline Q3 (0.75) & 8.0 & 6.2 & 6.3 & 5.8 & 6.6 \\
\hline Q1 (0.25) & 5.5 & 4.3 & 5.1 & 4.9 & 5.6 \\
\hline
\end{tabular}

Table 4.7 Statistics describing accuracy by presentation method

Figure 4.5 presents the accuracy of each presentation method. The wide variation for the direct and mirrored and side methods suggests that while many participants found the view helpful, others misinterpreted the view and replicated poses incorrectly. The mirrored view, mirrored and back view, and mirrored with feedback view all yield comparable levels of accuracy, but it is worth noting that the addition of a back view or feedback reduces the range of values. Additional information seems to help limit pose misinterpretation. 


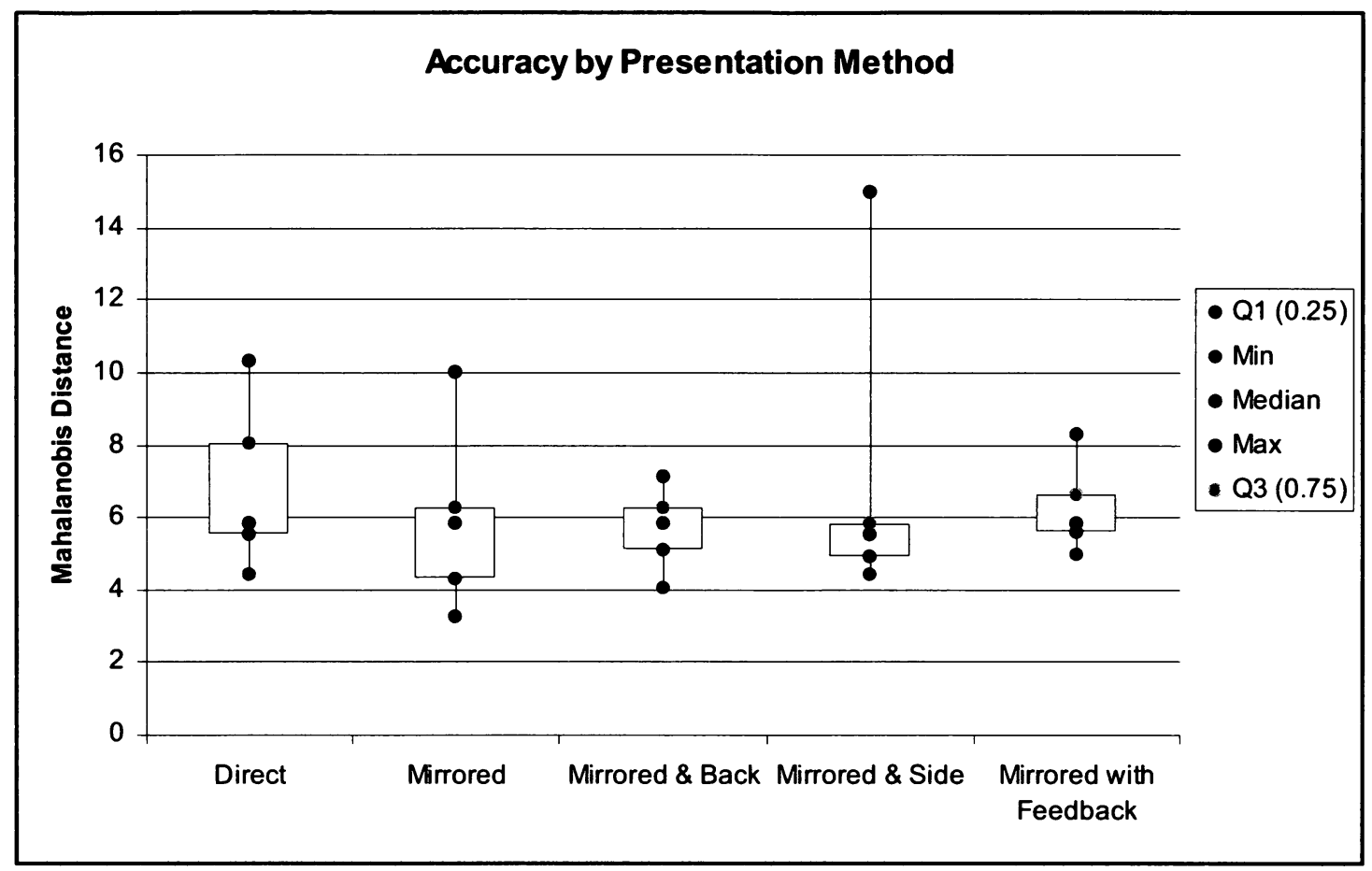

Figure 4.5 Mahalanobis distance by presentation method

Table 4.8 breaks down the pose accuracy, listing the average Mahalanobis distance per pose.

\begin{tabular}{|c|c|c|c|c|c|}
\hline \multirow{2}{*}{ Pose } & \multicolumn{5}{|c|}{ Distance } \\
\cline { 2 - 6 } & D & M & MB & MS & MF \\
\hline $\mathbf{1}$ & 6.0 & $\mathbf{4 . 9}$ & 5.6 & 5.3 & 7.4 \\
\hline $\mathbf{2}$ & 6.4 & 5.4 & 6.0 & $\mathbf{5 . 3}$ & 5.8 \\
\hline $\mathbf{3}$ & $\mathbf{5 . 0}$ & 5.6 & 5.8 & 8.9 & 6.7 \\
\hline $\mathbf{4}$ & 8.7 & 6.3 & $\mathbf{4 . 9}$ & 5.8 & 4.8 \\
\hline $\mathbf{5}$ & 8.3 & 6.8 & $\mathbf{5 . 6}$ & $\mathbf{5 . 6}$ & 6.0 \\
\hline
\end{tabular}

Table 4.8 Average Mahalanobis distance, broken down by individual pose

Figure 4.6 provides a direct comparison of the individual presentation methods on a perpose basis. 


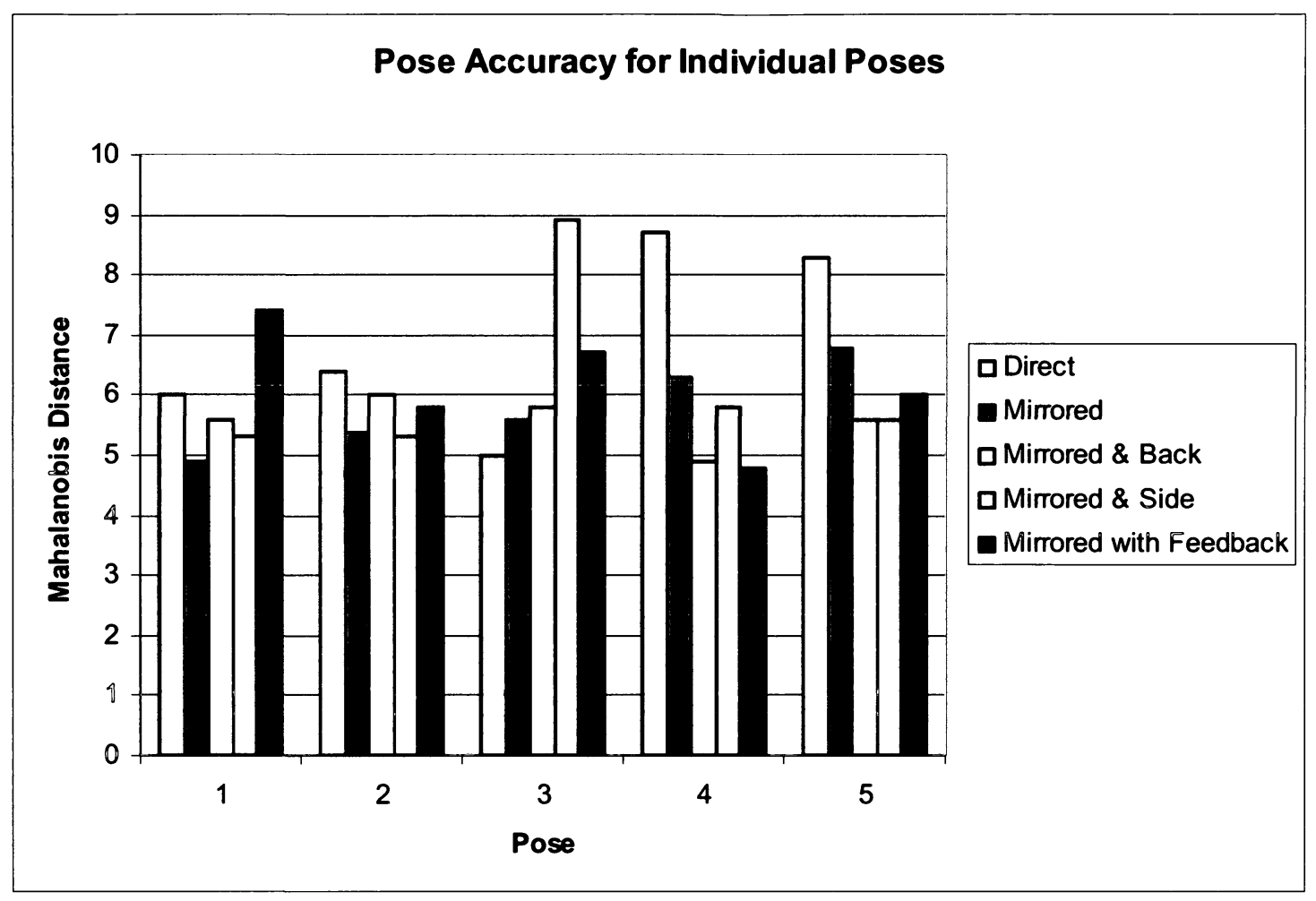

Figure 4.6 Breakdown of the accuracy of each pose

Pose 1 is symmetrical and physically unchallenging. Interestingly, the feedback method resulted in worse accuracy for this pose. Because the function used to provide feedback was relatively loose, participants received information that they were close to the correct pose, before they had made an effort to closely match the pose on screen. Distances for pose 2 were greater than pose 1 for all but the mirrored and side and mirrored with feedback views. Participants seemed to confuse the foot and leg positions, which were unclear from the straight-on view. For this pose, the additional back and side views were helpful in providing important depth cues. Pose 2 is shown below in Figure 4.7 from a mirrored, back, and side view to show the importance of an alternate view when the pose is not strictly planar. 

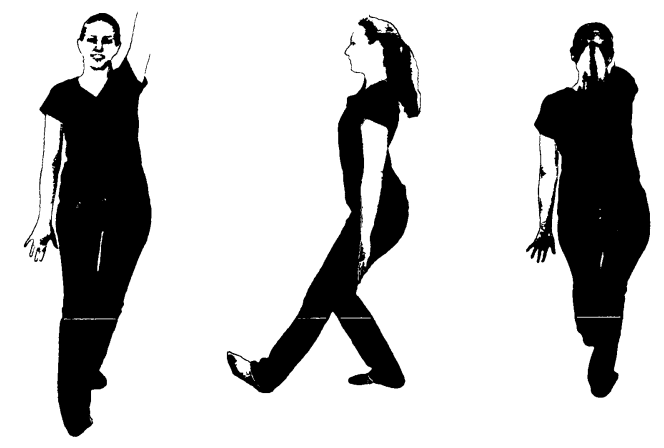

Figure 4.7 Pose 2 from the front (mirrored), side, and back views

Pose 3 is a relatively easy pose to replicate physically, however the asymmetry seems to be especially problematic for the mirrored and side view. This may be due in part to the combination of the mirrored and non-mirrored views. For this pose in particular, the side view provides the most detailed positional information, and consequently the most information about the pose, but it seems to be especially difficult for players to replicate poses from a sideways perspective. Pose 4 and 5 are more technically and physically complex. Additional information about the proper pose, from either the real-time feedback presentation method or additional views help users replicate the poses more accurately.

\subsection{Effect of Presentation Method on Perceived Difficulty}

The results of the survey for mental difficulty support the differences in speed for the direct, mirrored, mirrored and back and mirrored and side views. Participants were able to respond more quickly to the mirrored and mirrored and back views, and also reported fairly low mental difficulty. The opposite is true for the direct and mirrored and side views. The anomaly seems to be the mirrored and feedback view, which despite 
extremely slow response times, scored lowest on mental difficulty. Our hypothesis is that rather than spending additional time attempting to understand the presentation view, as is the case for direct and mirrored and side views, players are instead spending additional time perfecting their pose. The feedback provides players with valuable additional information that they are using to improve.

\begin{tabular}{|c|c|c|c|c|c|}
\hline & D & M & MB & MS & MF \\
\hline Average Perceived Mental Difficulty (1-5) & 2.5 & 1.8 & 1.8 & 2.4 & 1.6 \\
\hline Average Perceived Physical Difficulty (1-5) & 1.8 & 1.5 & 1.6 & 2.3 & 2.7 \\
\hline
\end{tabular}

Table 4.9 Average perceived mental and physical difficulty by presentation method

Self-reported data on perceived physical difficulty supports this conclusion. The mirrored and feedback view had the highest perceived difficulty physically replicating the poses. The poses were the same in all tests, however, in the four other conditions (direct, mirrored, mirrored and back, and mirrored and side views), players received no information as to the correctness of their poses. It was consequently easier for players to assume that the poses they performed were adequate, and thus, easy. The mirrored with feedback condition instead points out minor errors in body position, making the pose replication process seem more difficult. The opposite is true for the direct method, which results in high mental difficulty and comparably low perceived physical difficulty. As feedback adds to the perceived difficulty of the system, it should be adjusted to suit the accuracy requirements of the game. Where accuracy is less important, players should receive less demanding feedback. Feedback functions are described in the following section. 


\subsection{Pose Presentation Feedback Functions}

The mirrored with feedback presentation method may be easily modified to provide a variety of feedback, according to the needs of the system. A system emphasizing precision, for example, one that may be used in physiotherapy or training applications, may display more error, overlaying more red colour for smaller Mahalanobis distances. Alternatively, for a casual gaming application, it makes sense to present participants with red feedback only where their limb is in significant error. Any mathematical function may be used to provide users with precisely the kind of feedback necessary. It may be desirable to let players choose the nature of the feedback they receive in training, giving them several feedback function options. It should be noted that a certain amount of error is unavoidable and specifying a feedback function that requires players to replicate with extremely high accuracy may prove to be very frustrating. We characterize this minimum sensor placement error in the next section.

\subsection{Baseline Sensor Error}

As described in section 2.4.3, there is a certain amount of error that arises as a result of accelerometer noise and imprecise accelerometer placement on the body. Accelerometers may sit differently on different people's limbs. They can also shift around during, or even before, use. While measures can be taken to help participants place sensors accurately, it is unreasonable to expect that the sensors will be in the exact same location, at the exact same angle, for different participants. In fact, it is even unreasonable to expect a participant will place sensors in exactly the same location every time. 
While the standard deviations used in the Mahalanobis distance calculations allow for greater playability, they still do not allow players to reach certain levels of accuracy. The lowest Mahalanobis distance recorded in the presentation method test was 3.27 (for the mirrored method - see Table 4.7), with averages across presentation methods in the $5-7$ range. When used with the feedback presentation method, players become frustrated if the system requires extremely low Mahalanobis distance to remove coloured feedback. To address this issue, we propose a feedback function that first subtracts a baseline error for each sensor. A value of 1.0 per sensor is consistent with our findings in Table 2.4 and still requires an extremely high degree of pose precision, but could easily be modified based on the needs of the particular application.

\subsection{Dynamic Pose Presentation}

Each of the presentation methods examined provide users with different benefits. For some poses, a side view is especially helpful, but for others, an additional view is confusing. A solution may be to provide players with a view that dynamically adjusts based on the unique characteristics of individual poses. For example, the user could be presented with a view of the pose from the angle that captures the most positional detail. This limits the potential for occlusion of body parts and pose features that are critical to pose interpretation. Further testing is needed to determine whether the benefits of the additional information outweigh the mental challenges associated with re-orientation.

The concept of dynamic pose presentation could be extended further to provide players with even more information about specific hand or foot positions. In some poses, a wrist rotation may be critical to the correct interpretation of the pose, yet the subtlety may go 
unnoticed in a small, full-body image. An image overlay or other image processing to highlight specific pose features such as angles or positions may prove helpful. Arrows could be super-imposed on the pose image, indicating placement or direction. These features would, again, have to be tested to determine their benefit to players versus any possible distraction they might cause. While the mirrored presentation proved to be successful when players observe the pose straight-on, it may be confusing in other views. In a system that involves a dynamic, moving view point, it might be more effective to abandon the mirrored method entirely, as switching between the two types of view interpretation would likely be a source of confusion.

\subsubsection{Impact of Other Factors on Pose Success}

In this limited test case, no significant success correlations were found between fitness level, video game experience, height, or weight and pose speed or pose accuracy. No correlation was found between age and success within the 18-27 group. These results suggest that system training is suitable for diverse participants. It is possible, however, that fitness level and gaming experience may have an impact on overall player success in actual SNAP game play. For example, endurance helps players quickly and accurately replicate many poses when they are displayed in succession at faster rates. Age appears to be inversely correlated with both pose speed and accuracy among the older adult female group, however, the sample size is too small to make any definitive claims on the precise impact of age on either pose accuracy or speed. The tables below highlight the difference in average pose accuracy (Mahalanobis distances) for female participants of the varying age groups. 


\begin{tabular}{|c|c|c|}
\hline \multirow{2}{*}{ Age Group } & \multicolumn{2}{|c|}{ Speed (Seconds) } \\
\cline { 2 - 3 } & Mirrored & Mirrored with Feedback \\
\hline Females 18 - 27 & 4.4 & 8.6 \\
\hline Females 49 - 73 & 5.2 & 10.0 \\
\hline
\end{tabular}

Table 4.10 Speed of female participants of different ages

\begin{tabular}{|c|c|c|}
\hline \multirow{2}{*}{ Age Group } & \multicolumn{2}{|c|}{ Accuracy (Mahalanobis Distance) } \\
\cline { 2 - 3 } & Mirrored & Mirrored with Feedback \\
\hline Females 18- 27 & 5.4 & 6.1 \\
\hline Females 49-73 & 9.8 & 7.5 \\
\hline
\end{tabular}

Table 4.11 Accuracy of female participants of different ages

\subsubsection{Challenges Facing Older Participants}

Testing with the older adult female participants highlighted several key issues which may impact players of that demographic. Several participants had difficulty seeing the computer screen and positioned themselves closer to the computer than the younger participants. While it did not seem to impact their overall success rates, consideration should be given to placement and setup of the system if it is intended for older players. Two of the participants had to put the sensors on while seated. This in and of itself is not problematic for game play, but due to the elasticity of the sensor straps, the sensor pods required more subsequent adjustment. If the women were nervous or cautious about computer systems, sensors, or gaming systems, they did not show it! While there may be some additional issues impacting player performance, the participants were all extremely enthusiastic and made every effort to overcome the challenges. 


\section{Conclusion}

We began with a review of current exergames, both commercially available and prototype systems. This work is categorized by exercise type and further split into qualitative and quantitative studies. Brief reviews of persuasive technology and massively multiplayer online games are included to provide additional context for our work. We then introduced the Sensor Network for Active Play (SNAP) system, detailing its design and implementation, sensor pods and placement issues, pose detection, training and recognition strategies. Early experimental results highlight some of the strengths of the system, in particular, its capacity to elicit moderate physical activity levels, without negatively impacting player enjoyment. We introduce several SNAP game prototypes and the general design direction of the system.

Chapter 3 introduces a framework for a massively multiplayer online exergame (MMOE) to provide a customized, social gaming experience with the potential to provide players with real, long-term engagement and physical benefits. We describe key success factors for the MMOE, including the physical input system, motivation and enjoyment, tailoring and customization, and social factors. The strategies and critical features of the framework are summarized to facilitate future development and implementation of the system. We then assess the suitability of the SNAP system in the context of a massively multiplayer online game. The current version of SNAP meets the majority of the criteria set out by the framework as an effective input system, yielding positive physical benefits to players. Current SNAP game prototypes already leverage many motivational strategies and fun game elements. The structure of the dance-based games are particularly well- 
suited for future personalization. All of the SNAP game prototypes effectively leverage competition and social encouragement, but the social benefits inherent in an MMOG would complement the SNAP system even more effectively, providing the additional motivation and novelty necessary for players to remain engaged in on-going physical activity. As part of the analysis, we also suggest some additional modifications that may be made to SNAP in order to better meet the needs of an MMOE, such as wireless sensors, better feedback, and more opportunities for customization.

Finally, Chapter 4 outlines technical and usability challenges involved in the development of massively multiplayer online exergame with player-generated dance poses. Consideration is given to how players may best select their poses and we propose two alternative methods, one that aims to capture more serendipitous and spontaneous dance poses, and another which provides users with greater control over pose design. We subsequently discuss possible methods for pose capture, including a poseable avatar that players manipulate and a camera-network system. The advantages and disadvantages of the pose selection and capture methods are described, as the context of use ultimately determines the best method. Pose training in the context of an MMOE is not significantly different from the current dance-based SNAP game prototypes. The MMO system provides the added benefit of greater access to greater amounts of user pose data. When aggregate data sets fail to meet the needs of players, individuals can re-train poses.

To determine the most effective method of pose presentation, we conducted preliminary tests, comparing direct, mirrored, mirrored and back, mirrored and side, and mirrored 
with feedback views. In general, participants responded more quickly to mirrored versus direct presentation methods. The mirrored with feedback method resulted in the slowest response times and greatest perception of difficulty, which likely is a result of increased awareness of their inaccuracy. Accuracy results did not vary significantly among presentation methods, however the direct and mirrored and side views seemed to cause greater confusion, as indicated in a self-reported measure of perceived mental difficulty. The feedback method appears to be a promising strategy to use where accuracy is of greater importance than response speed (such as training applications), and offers many possibilities for refinement and customization. More complex and dynamic methods of pose presentation may provide an even more refined method of communicating poses in an MMOE context. Factors such as height, weight, fitness level, and gaming experience do not seem to impact the users' ability to replicate the poses. Testing with older adult females highlighted additional usability issues, emphasizing a need for easy system setup (including sensor placement) and accommodation of varying levels of visual acuity. In general, the older female participants performed the poses more slowly and less accurately than the 18-27 age group, but certainly not outside the range of usability and playability.

Future research will involve the implementation of the MMOE and distribution of the SNAP sensors to players in diverse locations for testing. Recommendations made for the MMOE framework require experimental verification and alternative strategies for the customizable pose creation system should also be implemented and tested. Minor design refinements may be made to the SNAP system for more effective use in the context of an 
MMOE, such as a switch to wireless sensors and variable sensor pod strap sizes. More complex and sophisticated methods of pose presentation could be developed and tested. Possibilities might include overlay screens, zoom-in detail highlights, or a 3-dimensional view with dynamic camera angles. Heart rate monitoring may be used as a proxy for activity level and as a means of more effectively demonstrating how similar systems can lead to improved fitness. Future work could also lead to new navigation strategies using only the SNAP system for input and control and eliminating the need for keyboard and mouse input for the MMOE. Long-term study of the physical and social effects of the MMOE system would also be an extremely valuable avenue for future work.

All trademarks and copyrights are the property of their respective owners 


\section{References}

[1] R. de Oliveira and N. Oliver, "TripleBeat: enhancing exercise performance with persuasion," Proceedings of the 10th international conference on Human computer interaction with mobile devices and services, Amsterdam, The Netherlands: ACM, New York, NY, 2008, p. 255-264.

[2] C. Garcia Wylie, C. Wylie, and P. Coulton, "Persuasive Mobile Health Applications," Electronic Healthcare: First International Conference, Ehealth 2008, London, September 8-9, 2008. Revised Selected Papers, Springer, 2009, p. 90.

[3] S. Arteaga, M. Kudeki, and A. Woodworth, "Combating obesity trends in teenagers through persuasive mobile technology," ACM SIGACCESS Accessibility and Computing, 2009, p. 17-25.

[4] J. Lin, L. Mamykina, S. Lindtner, G. Delajoux, and H. Strub, "Fish'n'Steps: encouraging physical activity with an interactive computer game," International Conference on Ubiquitous Computing, Springer, 2006, p. 261-278.

[5] W. Zhu, "Promoting physical activity through internet: a persuasive technology view," Proceedings of the Second International Conference on Persuasive Technology, Palo Alto, California, USA: Springer, 2007, p. 12.

[6] J. Lacroix, P. Saini, and A. Goris, "Understanding user cognitions to guide the tailoring of persuasive technology-based physical activity interventions," In Proceedings of the 4th International Conference on Persuasive Technology (Claremont, CA, USA, April, Claremont, California, USA: ACM, New York, NY, 2009, pp. 1-8.

[7] S. Berkovsky, D. Bhandari, S. Kimani, N. Colineau, and C. Paris, "Designing games to motivate physical activity," Proceedings of the 4th International Conference on Persuasive Technology, Claremont, California: ACM, New York, NY, 2009, pp. 1-4.

[8] "Wii at Nintendo," Nintendo of America Inc., 2009.

[9] B. Nardi and J. Harris, "Strangers and friends: Collaborative play in World of Warcraft," Proceedings of the 2006 20th anniversary conference on Computer supported cooperative work, ACM, 2006, pp. 149-158.

[10] N. Crampton, K. Fox, H. Johnston, and A. Whitehead, "Dance, Dance Evolution: Accelerometer Sensor Networks as Input to Video Games," HAVE 2007 - IEEE International Workshop on Haptic Audio Visual Environments and their Applications, Ottawa, Canada: IEEE, 2007, pp. 107-112.

[11] A. Whitehead, N. Crampton, K. Fox, and H. Johnston, "Sensor networks as video game input devices," Proceedings of the 2007 conference on Future Play - Future Play '07, New York, New York, USA: ACM New York, NY, 2007, pp. 38-45. 
[12] A. Whitehead, H. Johnston, K. Fox, N. Crampton, and J. Tuen, "Homogeneous Accelerometer-Based Sensor Networks for Game Interaction," ACM Computers in Entertainment, 2010.

[13] A. Whitehead, H. Johnston, N. Nixon, and J. Welch, "Exergame Effectiveness: What the Numbers Can Tell Us," Proceedings of the 2010 ACM SIGGRAPH Symposium on Video Games, 2010.

[14] J. Yim and T. Graham, "Using games to increase exercise motivation," Proceedings of the 2007 conference on Future Play, ACM, 2007, p. 166-173.

[15] D.E. Warburton, "The health benefits of interactive video game exercise," Applied Physiology, Nutrition, and Metabolism, vol. 32, 2007, pp. 655-663.

[16] S. Siegel, B. Haddock, A. Dubois, and L. Wilkin, "Active Video/Arcade Games (Exergaming) and Energy Expenditure in College Students," International Journal of Exercise Science, vol. 2, 2009.

[17] T. Stach, T.C. Graham, J. Yim, and R.E. Rhodes, "Heart Rate Control of Exercise Video Games," Graphics Interface Conference 2009, Kelowna, BC, Canada: ACM, 2009, pp. 125-132.

[18] J. Sinclair, P. Hingston, and M. Masek, "Considerations for the design of exergames," Computer graphics and interactive techniques in Australasia and South East Asia, 2007.

[19] M. Gardiner, "Physically Healthy Game Interfaces," cs.auckland.ac.nz, 2008, pp. $1-6$.

[20] A. Väätänen and J. Leikas, "Human-Centred Design and Exercise Games," Design and Use of Serious Games, Springer Netherlands, 2009, pp. 33-47.

[21] J. Höysniemi, "Design and Evaluation of Physically Interactive Games," Acta Electronica, 2006.

[22] S. Yang, B. Smith, and G. Graham, "Healthy Video Gaming: Oxymoron or Possibility," Journal of Online Education, vol. 4, 2006.

[23] "PlayStation 3 Accessories," Sony Computer Entertainment America Inc., 2010.

[24] R.R. Mellecker and A.M. McManus, "Energy expenditure and cardiovascular responses to seated and active gaming in children.," Archives of pediatrics \& adolescent medicine, vol. 162, 2008, pp. 886-91.

[25] C.G. Wylie and P. Coulton, "Mobile exergaming," Proceedings of the 2008 International Conference in Advances on Computer Entertainment Technology - ACE '08, vol. 44, 2008, p. 338. 
[26] M. Anders, "The Wii: As Good As the Real Thing?," American Council on Exercise; Fitness Matters, vol. 14, 2008, pp. 7-9.

[27] S. Cuzzort and T. Starner, "AstroWheelie: A wheelchair based exercise game," Proceedings of the 2008 12th IEEE International Symposium on Wearable ComputersVolume 00, IEEE Computer Society, 2008, p. 113-114.

[28] E. Charbonneau, A. Miller, C. Wingrave, and J. LaViola Jr, "Understanding visual interfaces for the next generation of dance-based rhythm video games," Proceedings of the 2009 ACM SIGGRAPH Symposium on Video Games, ACM, 2009, p. 119-126.

[29] R. Slyper and J. Hodgins, "Action capture with accelerometers," Proceedings of the 2008 ACM SIGGRAPH/Eurographics Symposium on Computer Animation, Eurographics Association, 2008, p. 193-199.

[30] C.N. Mhurchu, R. Maddison, Y. Jiang, A. Jull, H. Prapavessis, and A. Rodgers, "Couch potatoes to jumping beans: A pilot study of the effect of active video games on physical activity in children," International Journal of Behavioral Nutrition and Physical Activity, vol. 5, 2008, pp. 1-5.

[31] S. Consolvo, D. McDonald, T. Toscos, M. Chen, J. Froehlich, B. Harrison, P. Klasnja, A. LaMarca, L. LeGrand, and R. Libby, "Activity sensing in the wild: a field trial of ubifit garden," Proceedings of the Twenty-Sixth Annual SIGCHI Conference on Human Factors in Computing Systems, Florence, Italy: ACM, New York, NY, 2008, pp. 1797-1806.

[32] J. Hartnett, P. Lin, L. Ortiz, and L. Tabas, "A responsive and persuasive audio device to stimulate exercise and fitness in children," CHI'06 extended abstracts on Human factors in computing systems, ACM, 2006, p. 1842.

[33] B.J. Olmstead, "The effects of interactive video (DDR) on heart rate, perceived exertion, step count, self-efficacy, and enjoyment in elementary school children," 2006.

[34] S. Masuko and J. Hoshino, "A fitness game reflecting heart rate," Proceedings of the 2006 ACM SIGCHI international conference on Advances in computer entertainment technology - $A C E^{\prime}$ '06, New York, New York, USA: ACM Press, 2006, p. 53.

[35] R. Magielse and P. Markopoulos, "HeartBeat: an outdoor pervasive game for children," Proceedings of the 27th international conference on Human factors in computing systems, ACM, 2009, p. 2181-2184.

[36] A. Laikari, "Exergaming-gaming for health," The 13th IEEE International Symposium on Consumer Electronics (ISCE2009), IEEE, 2009, pp. 665-668.

[37] L. Gorgu, G.M. O'Hare, and M.J. O'Grady, "Towards Mobile Collaborative Exergaming," 2009 Second International Conference on Advances in Human-Oriented and Personalized Mechanisms, Technologies, and Services, 2009, pp. 61-64. 
[38] K. Kazakos, T. Bourlai, Y. Fujiki, and J. Levine, "NEAT-o-Games: novel mobile gaming versus modern sedentary lifestyle," MobileHCI 2008, Amsterdam, The Netherlands: ACM, 2008, pp. 515-518.

[39] B.A. Walker, L. Lanningham-Foster, J.A. Levine, T.B. Jensen, R.C. Foster, A.B. Redmond, and D. Heinz, "Energy expenditure of sedentary screen time compared with active screen time for children.," Pediatrics, vol. 118, 2006, pp. e1831-5.

[40] M.E. Willems and T.S. Bond, "Metabolic Equivalent of Brisk Walking and Playing New Generation Active Computer Games in Young-Adults," Medicina Sportiva, vol. 13, 2009, pp. 95-98.

[41] M. Pasch, N. Bianchi-Berthouze, B. van Dijk, and A. Nijholt, "Movement-based sports video games: Investigating motivation and gaming experience," Entertainment Computing, vol. 1, 2009, pp. 49-61.

[42] E.T. Khoo, A.D. Cheok, T.H. Nguyen, and Z. Pan, "Age invaders: social and physical inter-generational mixed reality family entertainment," Virtual Reality, vol. 12, 2008, pp. 3-16.

[43] M. Pasch, N. Berthouze, B.V. Dijk, and A. Nijholt, "Motivations, Strategies, and Movement Patterns of Video Gamers Playing Nintendo Wii Boxing," Facial and Bodily Expressions for Control and Adaptation of Games (ECAG 2008), A. Nijholt and R.W. Poppe, Centre for Telematics and Information Technology, University of Twente, 2008, pp. 27-33.

[44] F. Mueller, S. Agamanolis, M. Gibbs, and F. Vetere, "Remote impact: shadowboxing over a distance," Proceedings of the 27th international conference extended abstracts on Human factors in computing systems, ACM, 2009, p. 3531-3532.

[45] J. Hoysniemi, "International survey on the Dance Dance Revolution game," Computers in Entertainment, vol. 4, 2006, p. 8.

[46] V.B. Unnithan, W. Houser, and B. Fernhall, "Evaluation of the energy cost of playing a dance simulation video game in overweight and non-overweight children and adolescents.," International journal of sports medicine, vol. 27, 2006, pp. 804-9.

[47] D. Warburton, D. Sarkany, M. Johnson, R. Rhodes, W. Whitford, B. Esch, J. Scott, S. Wong, and S. Bredin, "Metabolic requirements of interactive video game cycling," Medicine \& Science in Sports \& Exercise, vol. 41, 2009, p. 920.

[48] L.E. Graves, N.D. Ridgers, and G. Stratton, "The contribution of upper limb and total body movement to adolescents' energy expenditure whilst playing Nintendo Wii.," European journal of applied physiology, vol. 104, 2008, pp. 617-23.

[49] M. Gibbs, "A physical three-way interactive game based on table tennis," Proceedings of the 4th Australasian conference on Interactive entertainment, RMIT University Melbourne, Australia, Australia, 2007. 
[50] F. Mueller, M.R. Gibbs, and F. Vetere, "Design influence on social play in distributed exertion games," Proceedings of the 27th international conference on Human factors in computing systems - CHI '09, 2009, p. 1539.

[51] T. Toscos, A. Faber, S. An, and M. Gandhi, "Chick clique: persuasive technology to motivate teenage girls to exercise," CHI'06 extended abstracts on Human factors in computing systems, Montréal, Québec, Canada: ACM New York, NY, 2006, pp. 18731878.

[52] F. Mueller, S. Agamanolis, F. Vetere, and M. Gibbs, "Brute force as input for networked gaming," Proceedings of the 2007 conference of the computer-human interaction special interest group (CHISIG) of Australia on Computer-human interaction: design: activities, artifacts and environments - OZCHI '07, 2007, p. 167.

[53] S. Consolvo, K. Everitt, I. Smith, and J. Landay, "Design requirements for technologies that encourage physical activity," Proceedings of the SIGCHI conference on Human Factors in computing systems, Montréal, Québec, Canada: ACM, New York, NY, 2006, p. 466.

[54] T. Campbell, B. Ngo, and J. Fogarty, "Game design principles in everyday fitness applications," Proceedings of the ACM 2008 conference on Computer supported cooperative work - CSCW'08, 2008, p. 249.

[55] M.J. Chin A Paw, W.M. Jacobs, E.P. Vaessen, S. Titze, and W. van Mechelen, "The motivation of children to play an active video game.," Journal of science and medicine in sport / Sports Medicine Australia, vol. 11, 2008, pp. 163-6.

[56] L. Graves, G. Stratton, N.D. Ridgers, and N.T. Cable, "Comparison of energy expenditure in adolescents when playing new generation and sedentary computer games: cross sectional study," BMJ (Clinical research ed.), vol. 335, 2007, pp. 1282-4.

[57] F. Buttussi, L. Chittaro, R. Ranon, and A. Verona, "Adaptation of graphics and gameplay in fitness games by exploiting motion and physiological sensors," Proceedings of the 8th international symposium on Smart Graphics, Kyoto, Japan: Springer-Verlag, 2007, pp. 85-96.

[58] E. Kalapanidas, C. Davarakis, F. Fernández-Aranda, S. Jiménez-Murcia, O. Kocsis, T. Ganchev, H. Kaufmann, T. Lam, and D. Konstantas, "PlayMancer: Games for Health with Accessibility in Mind," Communications \& Strategies, 2009, pp. 105-120.

[59] B. Chamberlain and R. Gallagher, "Exergames: Using Video Games to Promote Physical Activity," Children, Youth and Families at Risk (CYFAR), San Antonio, TX, USA: 2008, pp. 3-6.

[60] M. Papastergiou, "Exploring the potential of computer and video games for health and physical education: A literature review," Computers \& Education, vol. 53, 2009, pp. 603-622. 
[61] U. Dieterle, "Games/Simulations for Health: Tracking Down the Evidence on Efficacy," 25th Annual Conference on Distance Teaching \& Learning, 2009, pp. 1-4.

[62] D. Graf, L. Pratt, C. Hester, and K. Short, "Playing active video games increases energy expenditure in children," Pediatrics, vol. 124, 2009, pp. 534-540.

[63] J.A. McWha and G.A. Brown, "Effects of energy expenditure while playing the Nintendo Wii against a human and computer opponent."

[64] L. Lanningham-Foster, R.C. Foster, S.K. McCrady, T.B. Jensen, N. Mitre, and J.A. Levine, "Activity-promoting video games and increased energy expenditure," The Journal of pediatrics, vol. 154, 2009, pp. 819-23.

[65] K. Sell, T. Lillie, J. Taylor, J. Vener, L. Ransdell, and C. Tudor-Locke, "Quantifying Upper Body Physical Activity During Interactive Video Gaming for College Students," Medicine \& Science in Sports \& Exercise, vol. 37, 2005.

[66] K. Sell, T. Lillie, and J. Taylor, "Energy Expenditure during physically interactive video game playing in male college students with different playing experience," Journal of American College Health, vol. 56, 2008, pp. 505-511.

[67] R. Maddison, C. Mhurchu, A. Jull, Y. Jiang, H. Prapavessis, and A. Rodgers, "Energy expended playing video console games: an opportunity to increase children's physical activity?," Pediatric exercise science, vol. 19, 2007, p. 334.

[68] T. Lillie, K. Sell, J. Taylor, J. Vener, L. Ransdell, and C. Tudor-Locke, "Physical Activity Recommendations Can Be Met Using A Physically Interactive Video Game Among College Students," Medicine \& Science in Sports \& Exercise, vol. 37, 2005.

[69] R.R. Mellecker, A.M. McManus, L.M. Lanningham-Foster, and J.A. Levine, "The feasibility of ambulatory screen time in children," International Journal of Pediatric Obesity, vol. 4, 2009, pp. 106-111.

[70] L. Keytel, J. Goedecke, T. Noakes, H. Hiiloskorpi, R. Laukkanen, L. Van Der Merwe, and E. Lambert, "Prediction of energy expenditure from heart rate monitoring during submaximal exercise," J SPorts Sci, vol. 23, 2005, pp. 289-97.

[71] T. Stac, T.C. Graham, M. Brehmer, and A. Hollatz, "Classifying input for active games," Proceedings of the International Conference on Advances in Computer Entertainment Technology - ACE '09, New York, New York, USA: ACM Press, 2009, p. 379.

[72] "Wii MotionPlus at Nintendo," Nintendo of America Inc., 2010.

[73] "Xbox.com - Project Natal," Microsoft Corporation.

[74] M. Furtado, "Project Natal Struggles with Dark Skinned People," examiner.com, 2010 . 
[75] E.B. Wassenaar and J.G. Van den Brand, "Reliability of near-infrared spectroscopy in people with dark skin pigmentation.," Journal of clinical monitoring and computing, vol. 19, 2005, pp. 195-9.

[76] B. Fogg, Persuasive Technology: Using Computers to Change What We Think and Do, Morgan Kaufmann, 2003.

[77] T.M. Bekker and B.H. Eggen, "Designing for children's physical play," Proceeding of the twenty-sixth annual CHI conference extended abstracts on Human factors in computing systems - CHI'08, 2008, p. 2871.

[78] C. Klimmt and T. Hartmann, "Effectance, self-efficacy, and the motivation to play video games.," Playing Video Games: Motives, Responses, and Consequences, P. Vorderer and J. Bryant, Mahwah, NJ: Lawrence Erlbaum Associates, 2006, pp. 143-177.

[79] D.A. Lieberman, "What can we learn from Playing Interactive Games?," Playing Video Games: Motives, Responses, and Consequences, P. Vorderer and J. Bryant, Mahwah, NJ: Lawrence Erlbaum Associates, 2006, pp. 379-397.

[80] A. Raney, J.K. Smith, and K. Baker, "Adolescents and Video Games," Playing Video Games: Motives, Responses, and Consequences, P. Vorderer and J. Bryant, Mahwah, NJ: Lawrence Erlbaum Associates, 2006, pp. 191-209.

[81] J. Bryant and J. Davies, "Selective Exposure to Video Games," Playing Video Games: Motives, Responses, and Consequences, P. Vorderer and J. Bryant, Mahwah, NJ: Lawrence Erlbaum Associates, 2006, pp. 210-228.

[82] M. Csikszentmihalyi, Flow: The Psychology of Optimal Experience, Harper Perennial, 1991.

[83] P. Vorderer and J. Bryant, Playing Video Games: Motives, Responses, and Consequences, Lawrence Erlbaum Associates, 2006.

[84] S.M. Zehnder and S.D. Lipscomb, "The Role of Music in Video Games," Playing Video Games: Motives, Responses, and Consequences, P. Vorderer and J. Bryant, Mahwah, NJ: Lawrence Erlbaum Associates, 2006, pp. 241-258.

[85] D. Priest, C. Karageorghis, and N. Sharp, "The characteristics and effects of motivational music in exercise settings: the possible influence of gender, age, frequency of attendance, and time of attendance.," The Journal of sports medicine and physical fitness, vol. 44, 2004, p. 77.

[86] R. Khaled, P. Barr, R. Biddle, R. Fischer, and J. Noble, "Game design strategies for collectivist persuasion," Proceedings of the 2009 ACM SIGGRAPH Symposium on Video Games, ACM, 2009, p. 31-38. 
[87] A. Axelsson and T. Regan, "Playing Online," Playing Video Games: Motives, Responses, and Consequences, P. Vorderer and J. Bryant, Mahwah, NJ: Lawrence Erlbaum Associates, 2006, pp. 291-306.

[88] V.P. Chan E., "Massively Multiplayer Online Games," Playing Video Games: Motives, Responses, and Consequences, P. Vorderer and J. Bryant, Mahwah, NJ: Lawrence Erlbaum Associates, , pp. 88-103.

[89] "Second Life Official Site," Linden Research, 2010.

[90] "Disney's Toontown Online," Disney, 2010.

[91] "The Sims," Electronic Arts Inc., 2007.

[92] "Dance! Online," Acclaim Games Inc., 2008.

[93] R.O. Duda, P.E. Hart, and D.G. Stork, Pattern Classification (2nd Edition), Wiley-Interscience, 2000.

[94] P. Mahalanobis, "On the generalised distance in statistics," vol. 2, 1936, pp. 49 55.

[95] B. Ling and S.S. Intille, "Activity recognition from user-annotated acceleration data," Lecture notes in computer science, 2004, pp. 1-17.

[96] A. Whitehead and K. Fox, "Device agnostic 3D gesture recognition using hidden Markov models," Proceedings of the 2009 Conference on Future Play on @ GDC Canada, Vancouver, British Columbia, Canada: ACM, 2009, pp. 29-30. 